\title{
A Sustainable Preparation of Functional Perylenophanes by Domino Metathesis
}

\author{
Heinz Langhals*, Maximilian Rauscher, Peter Mayer \\ Department of Chemistry, LMU University of Munich, Munich, Germany \\ Email: *Langhals@lrz.uni-muenchen.de
}

How to cite this paper: Langhals, $\mathrm{H}$., Rauscher, M. and Mayer, P. (2019) A Sustainable Preparation of Functional Perylenophanes by Domino Metathesis. Green and Sustainable Chemistry, 9, 38-77. https://doi.org/10.4236/gsc.2019.92004

Received: March 10, 2019

Accepted: May 28, 2019

Published: May 31, 2019

Copyright $\odot 2019$ by author(s) and Scientific Research Publishing Inc. This work is licensed under the Creative Commons Attribution International License (CC BY 4.0).

http://creativecommons.org/licenses/by/4.0/

(c) (i) Open Access

\begin{abstract}
A sustainable four-step synthesis of soluble perylenophanes for applications as fluorescent optical functional materials is presented and even allows upscaling because of starting with technical bulk products. Thus, terminal alkenylnitriles were alkylated reduced to amines, condensed with perylenetetracarboxylic bisanhydride and cyclised to cyclophanes by means of double cross metathesis in yields until $69 \%$ of isolated dyes. The first metathesis by means of the second-generation Hoveyda-Grubbs-catalyst brings the remaining reactive olefinic groups close together favouring the ring-closure to the cyclophanes where the locked neighboring of chromophores in a skew arrangement induce strong exciton interactions. The latter cause an increased the Stokes' separation by means of a moderate hypsochromic shift of light absorption and a stronger bathochromic shift of fluorescence. Various applications such as for lasers, photonics, solar collectors or in analytics are discussed.
\end{abstract}

\section{Keywords}

Optical Materials, Functional Dyes, Fluorescence, Exciton Interaction, Metathesis

\section{Introduction}

The interaction of two or more chromophores offers many possibilities for modern optoelectronics [1] and functional materials because various optical effects can be generated where the perylene dyes [2] such as 1 [3] and other pe$r i$-arylenes [4] are suitable components because of their high chemical and photochemical stability and fluorescence quantum yields. Moreover, one single optical transition of these chromophores in the visible spectral region favors the planning, construction and operation of functional multichromophoric assemblies. The elec- 
tronic interaction of two chromophores induces spectral shifts of the initial absorption to two new positions known as the Davydov splitting [5] [6]. Thus, a more bathochromic $\alpha$-transition and a more hypsochromic $\beta$-transition are obtained instead of the light absorption of the individual chromophores; see Figure 1, left.

There are two fundamental arrangements of the transition moments of two or more chromophores with (i) coplanarity known as $H$-orientation [7] according to the initially described hypsochromically absorbing aggregates and (ii) linear shift known as $J$-arrangement [8]; see Figure 1, right.

The $H$-orientation induces an anti-synchronous electron movement according to Förster's analysis [9] because of Coulomb interactions; this is schematically indicated in Figure 1 with an arbitrary flashlight distribution of charges. The electrostatic interactions remain still somewhat disfavorable because the comparably short distance of equal charges; thus, the transition energy of the individual chromophores increases for the $H$ orientation and causes a hypsochromic shift of light absorption. The $H$-arrangement lowers the molar absorptivity because of an effective shortening the compact molecular antenna for two chromophores. Moreover, the point symmetry compensates the transition moment and thus, suppresses fluorescence. The $J$-arrangement with linearly shifted chromophores induces a synchronous electron movement with more favorable attractive Coulomb interactions, lowers the transition energy, causes a bathochromic shift, increases the molar absorptivity because the extended antenna and allows fluorescence.

A skew-type arrangement of chromophores activates both transitions so that

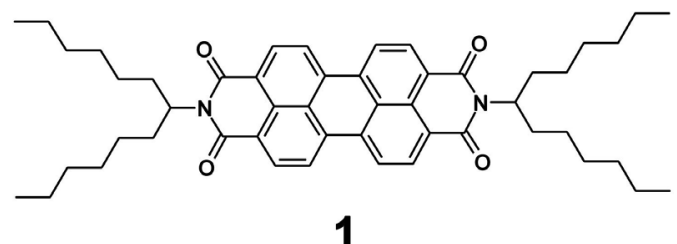

1
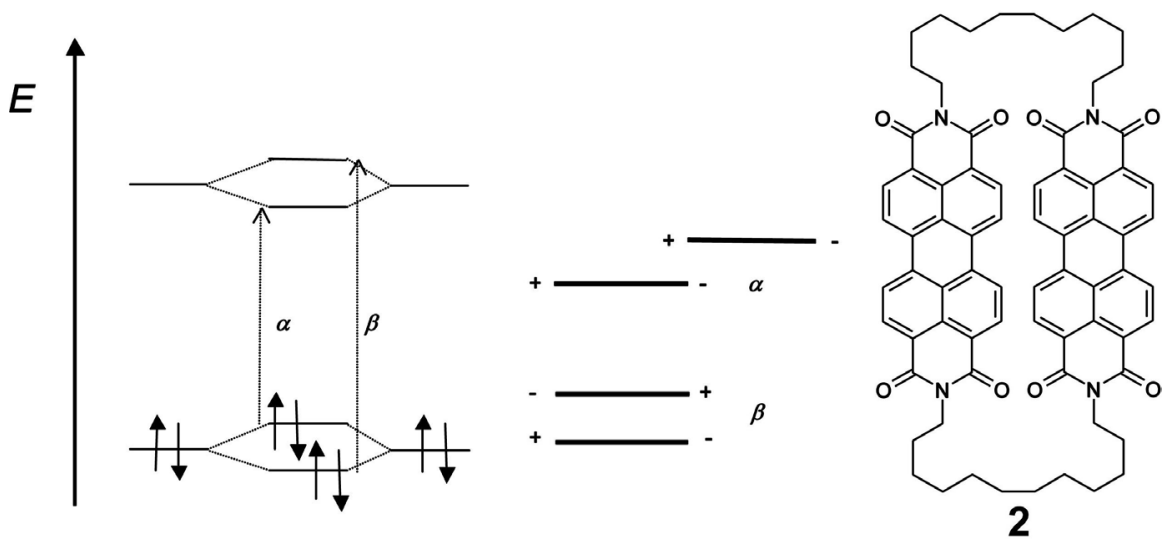

Figure 1. Left: Energy levels of two interacting chromophores and resulting Davydov splitting in the middle with the resulting electronic $\alpha$ and $\beta$ transitions. Middle: Basic orientations of transition moments, coplanar in $H$ (bottom, $\beta$ ) and linearly shifted in $J$ arrangements (top, $\alpha$ ). Indication of a momentaneous charge distribution by “+” and “-”. Right: Formula 2. 
the initial absorption of the isolated chromophores appears to be split in two bands: Davydov splitting. The intensities of the individual bands depend from the exact orientation of the transition moments of the involved chromophores.

Aggregates such as $H$ and $J$ arrangement of chromophores are only weakly held together by non-covalent interactions and are labile concerning dissociation such as with dilution. A higher stability can be obtained with covalently linked chromophores, preferently forming cyclophanes such as 2 [10]. However, synthesis of such cyclophanes proved to be complicated for complex chromophores and upscaling of $\mathbf{2}$ is difficult because of simultaneous and stoichiometric addition of two solids under dilution conditions. Moreover, the limited solubility of 2 means an obstacle for applications in homogeneous media. An efficient method for the preparation of such cyclophanes with increased solubility would bring about appreciable progress.

\section{Results and Discussions}

Here we applied olefin metathesis [11] [12] [13] [14] [15] for the efficient, economic and sustainable synthesis of bichromophoric perylenetetracarboxylic bisimides dyes (dyads) because of catalysed carbon-carbon linking where special optic effects are expected as a consequence of the interacting chromophores in such molecules. Firstly, we studied the linking of simple alkylene-substituted perylene carboxamides by metathesis; however, the generally very low solubility of perylenebiscarboximides means an obstacle for such bimolecular reactions. Thus, we introduced solubility-increasing groups and firstly attached the 2,5-di-tert-butyl substituent [2] to one nitrogen atom of perylenbiscarboximide and an allyl group as the terminal olefine to the other; the nitrogen atoms form ideal positions for the ring-formation because of orbital nodes [16] in HOMO and LUMO causing an electronic decoupling of substituents at these positions. However, metathesis with second-generation Hoveyda-Grupps-catalyst (4) [17] gave only low yields of a few percents. Obviously, the solubility of the staring material is still too low for efficient coupling.
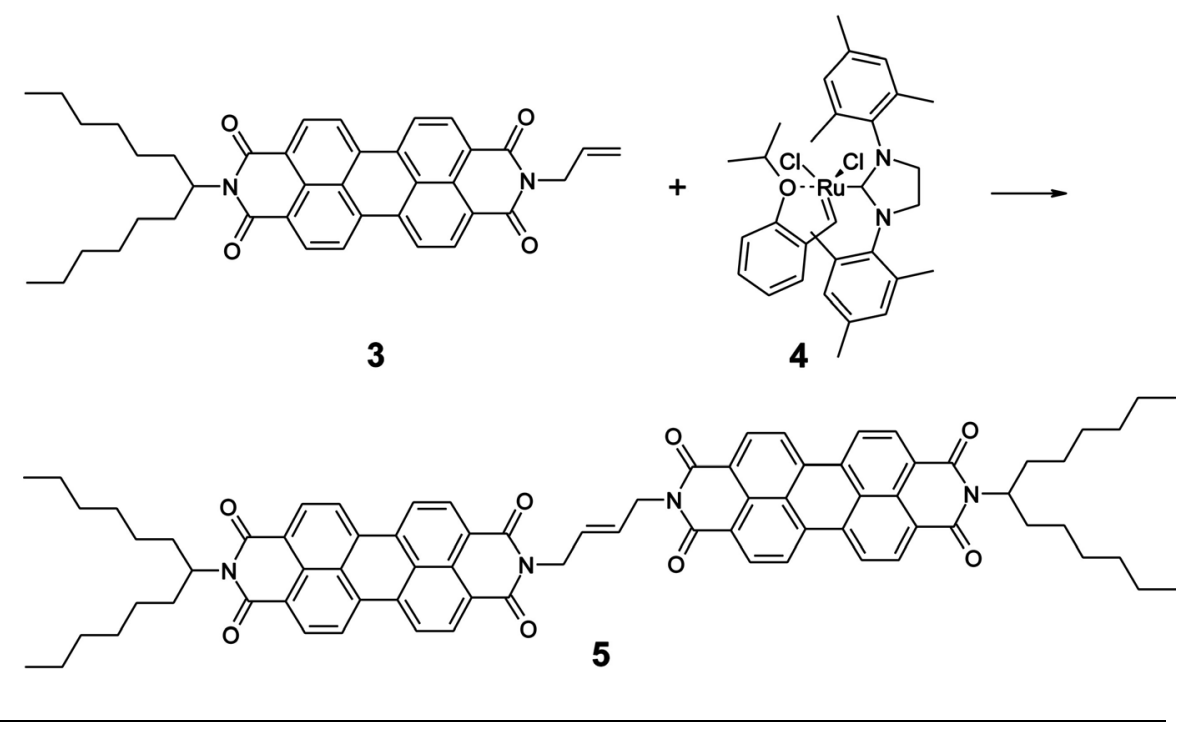
As a consequence, we introduced the more efficiently solubilising 7-tridecyl group in 3 and obtained $10 \%$ of the dyad $\mathbf{5}$ as a cis/trans mixture as was also found for all other products of metathesis (only the trans isomer of 5 is shown).


10



The analogous coupling of the 4-vinylphenyl derivative 6 for the generation of the more rigid stilbene as the spacer gave higher yields; however, a sparingly soluble pigment-like material $\mathbf{7}$ was obtained with limited use for investigation and application in homogeneous solution. A prolongation of the swallow-tail substituents to octylnonyl (6a and $7 \mathbf{a}$ ) and further to nonyldecyl (6b and $7 \mathbf{b}$ ) did not sufficiently improve the solubility. Furthermore, we introduced geminal alkyl 


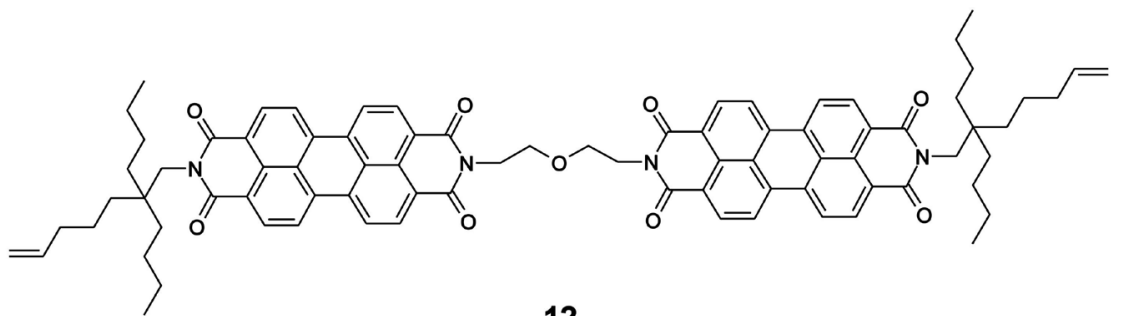

12
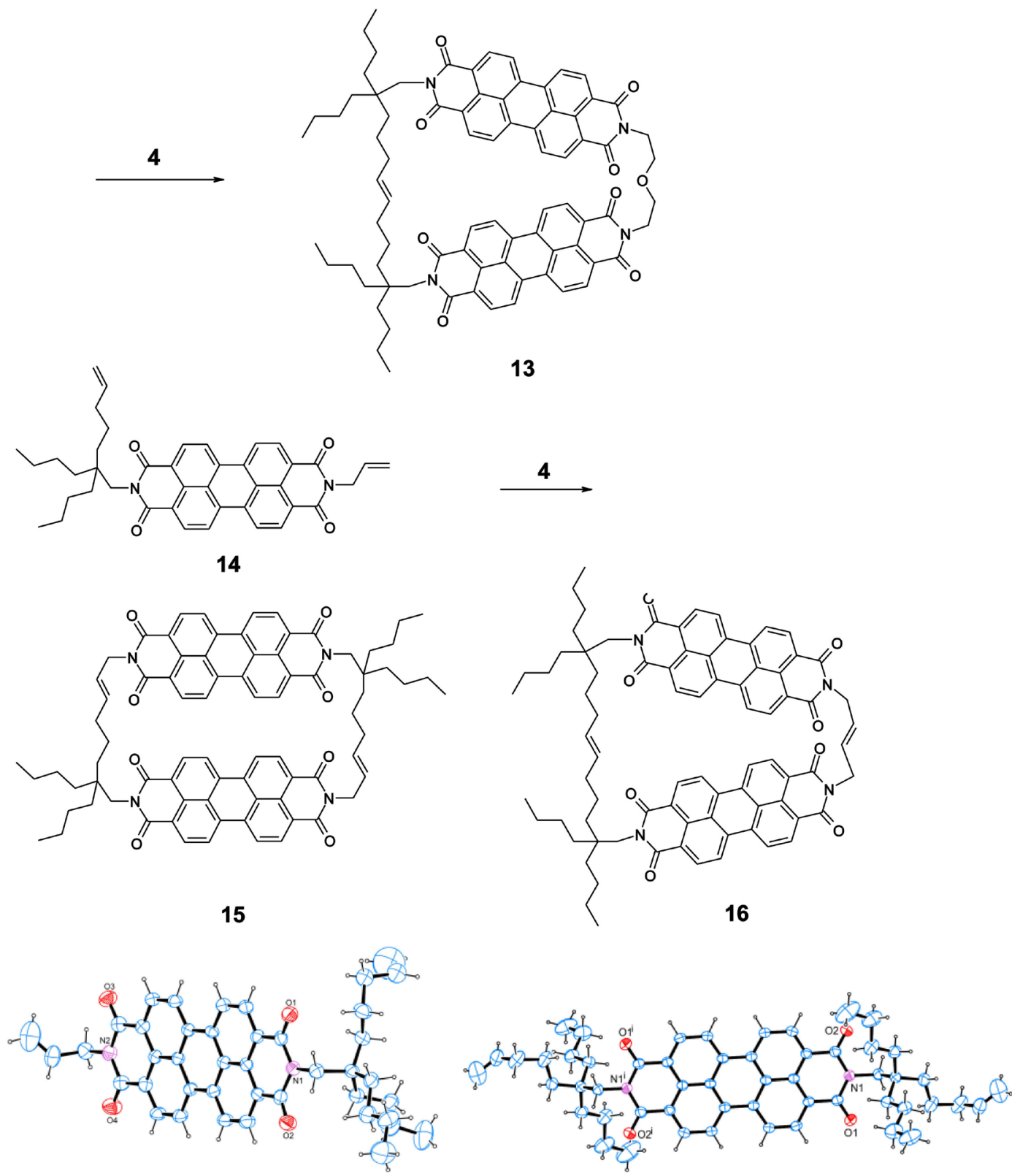

Figure 2. X-ray crystal structure analyses. Left: The starting material 14 for 15 and 16 (kn237). Right: The starting material 21 for 22 (kn095).

groups [18] in 8 into the linker between the two chromophores and an even longer chain in 10 and could improve the solubility by the prolongation of the interlinking chain in 9 and 11, respectively; however, such compounds exhibit 
an unexpected and for 11 even more pronounced tendency of the degradation of the central connecting chain as a consequence of the contact to air. Resuming, the coupling of the simple allyl derivative 3 with a 7 -tridecyl group at one nitrogen atom gave best results and indicates that metathesis is generally applicable for the synthesis of dyads. As a consequence, we investigated ring-closing metathesis because of higher proximity of the reacting olefinic groups.

Thus, we firstly connected in 12 two chromophores with an $N$-hydroxyethyl group by means of etherification in a one-pot reaction with methanesulphonic acid chloride. The nitrogen atoms at the opposite sides of the chromophores were attached to terminal olefinic alkyl groups where geminal alkyl substituents were applied for solubility increasing. Ring-closing metathesis to 13 was realised by means of second-generation Hoveyda-Grupps-catalyst (4); however, the solubility of the material was still comparably restrainingly low. Moreover, two steps with moderate yields were necessary for the preparation of a cyclophane. For further improvement, we targeted two consecutive metatheses as a Domino reaction in one pot and replaced the hydroxyethyl group in the starting material for 13 by an allyl group in 14; for structural details see Figure 2, left. However, a direct metathesis gave a complex mixture of products containing 15 and 16 both as cis/trans mixtures. As a consequence, we allowed to react the symmetrically substituted perylene dyes 17,19 and 21 with $N, N^{*}$-2-butyl-2- $\omega$-alkenylpentyl groups in metathesis with $\mathbf{4}$ and obtained the corresponding for applications sufficiently soluble cyclophanes 18, 20 and 22 as cis/trans mixtures with very similar properties; for structural details of the starting material 21 for 22 , see Figure 2, right. Comparably satisfying yields of cyclophanes were obtained as high as $69 \%$ of 22 . No polymeric material could be detected indicating the favoring of the second metathesis as a consequence of the neighbourship of the chromophores after the first metathesis.

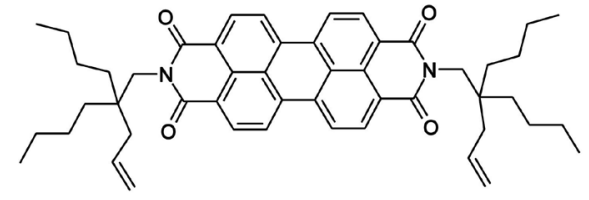

17

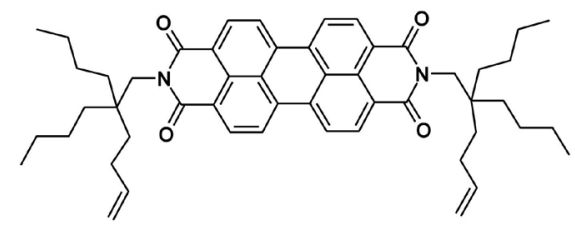

19

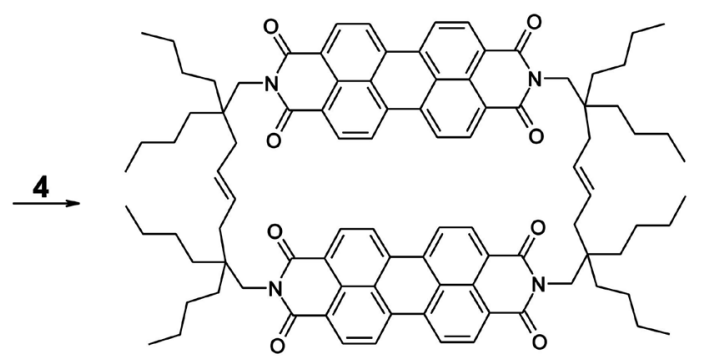

18

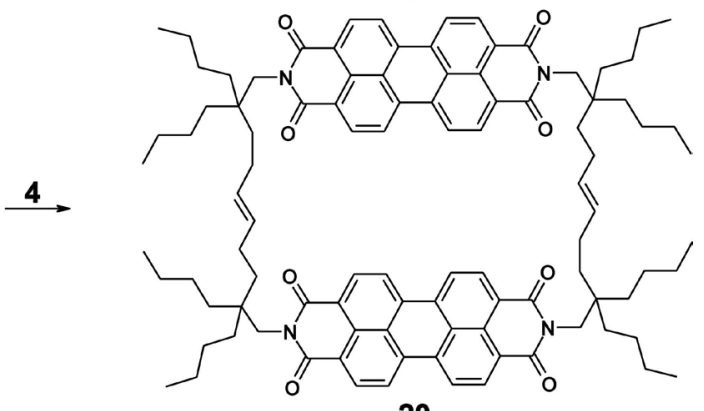

20 

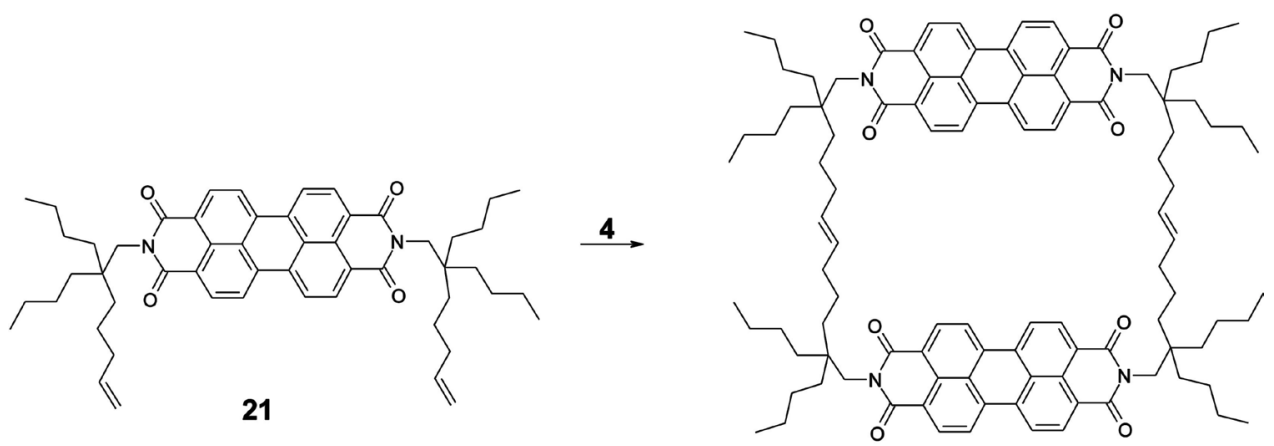

22

The yields of isolated pure material of such Domino reactions increase with the flexibility of the generated cyclophanes where 50\% were obtained for the compact 18, 57\% for 20 with a larger mash and even $69 \%$ for 22 with a still larger ring. A prolongation of the alkyl chains in the swallow-tail system or a replacement by iso-propyl groups appreciably decreases the yields of cyclophanes presumably caused by a less compact arrangement for the second and ring-closing metathesis as a Domino reaction. Resuming, the cyclophanes 18, 20, and 22 are preferred for special optical applications. As a consequence, a sustainable, efficient, economic and upscalable access to the starting materials $\mathbf{2 3}$ for metathesis would bring about further progress.

Thus, we started synthesis with the alkylation [19] of $\omega$-akenyl nitriles with 1-iodobutane by means of LDA (lithium diisopropylamide) in a laboratory synthesis according to Figure 3; an efficient upscaling is possible applying alkyl bromides with sodium and sodium amide, respectively, in liquid ammonia according to Ref. [20] in a solvent-recycling chain of batches [21]. The alkylated nitrile was reduced with complex hydrides such as lithium aluminiumhydride or even catalytic with hydrogen to the primary amine and the latter condensed with the technical mass product perylene-3,4:9,10-tertacarboxylicbisahydride in melt imidazole [22] [23] with the addition of zinc acetate to obtain 23 with $n=1,2$ and 3 .

Special optic effects [9] are expected as a consequence of the interaction of chromophores [24] in the dyads (bichromophores). However, the UV/Vis spectra of the open-chain dyad 5 are nearly identic with the spectra of 1 (Figure 4, left spectrum, red and green curves). Obviously, the long distance of the individual chromophores allows only slight exciton interactions [25]. Thus, a more tight arrangement of the chromophores was targeted for stronger interactions. Two chromophores linked with an ether group and tied close together by ring-closing

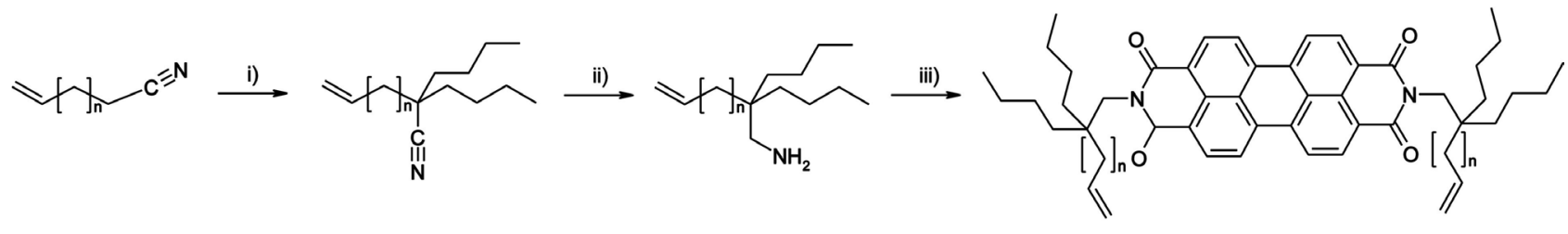

23

Figure 3. Synthesis of the starting materials for metathesis: $23, n=1$ until 3 by means of alkylation of terminal alkenyl nitriles, reduction and condensation. i) $\mathrm{C}_{4} \mathrm{H}_{9} \mathrm{I}$, LDA; ii) $\mathrm{LiAlH}_{4}$; iii) Perylene-3,4:9,10-tetracarboxylicbisanhydride, zinc acetate, imidazole. 


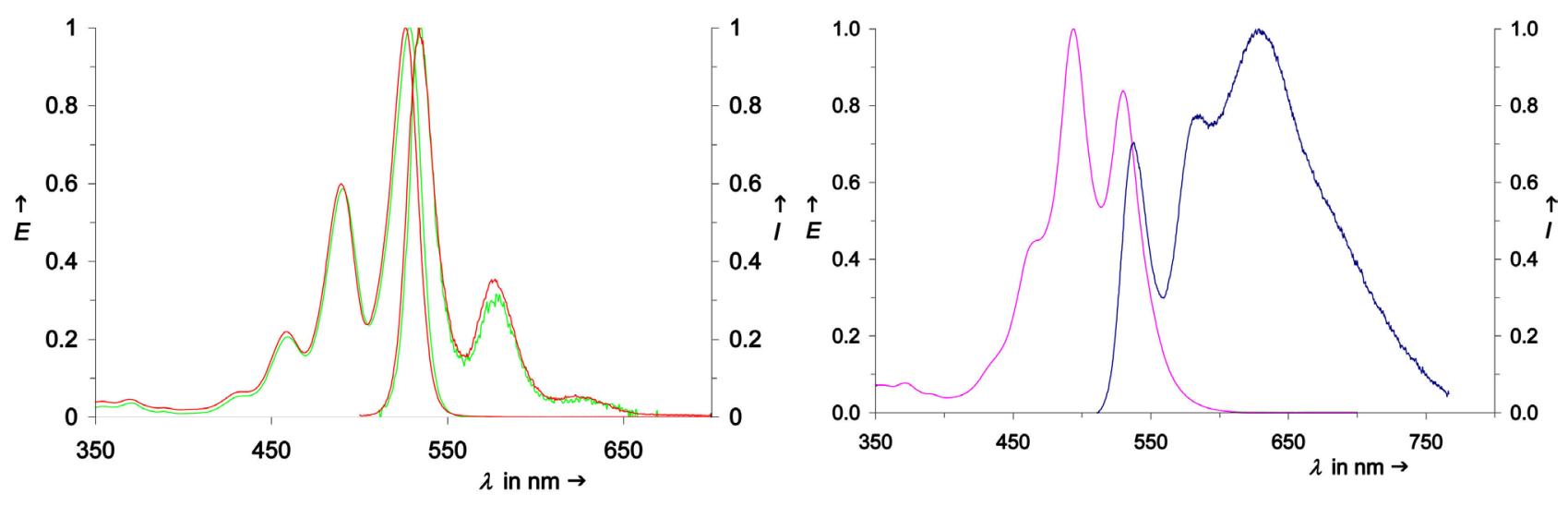

Figure 4. Left spectrum: UV/Vis absorption (left) and fluorescence (right) spectra in chloroform. Green curves: 5, red cuves: $\mathbf{1}$ for comparison. Right spectrum: UV/Vis absorption (left, magenta) and fluorescence (right, blue) spectra of 13 in chloroform.

metathesis in the cyclophane 13 form a skew arrangement where there are optical transitions with a major $H$-component (hypsochromic $\beta$ transition) and a minor J-component (bathochromic $\alpha$ transition); compare Ref. [26] for similar arrangements in micelles. As a consequence, the more hypsochromically absorbing $\beta$ transition dominates the absorption spectrum (Figure 4, right spectrum, magenta curve, left, more hypsochromic compared with the red curve of 1 in the left spectrum); however, fluorescence is suppressed because of symmetry according to Förster's [9] analysis. On the other hand, the minor J-component with more bathochromic transitions allows fluorescence and causes the broad fluorescence spectrum at longer wavelengths shown in Figure 4 (right spectrum, blue curve, right). Overall, the Stokes' shift of 13 is increased compared with 1; however, the still comparably low solubility of 13 means still an obstacle for many optoelectronic applications. Moreover, the two-step ring-closure is unfavorable for the overall yield. As a consequence the products 15 and 16 of a double one pot metathesis (domino metathesis) both as mistures of cis/trans isomers were investigated as an alternative.

15 and 16 could be separated by means of preparative T.L.C. and gave the surprising result of nearly identic UV/Vis absorption and fluorescence spectra although the chomophore-linking chains are different; see Figure 5, left spectrum. Moreover, the spectra resemble 13 both in absorption and fluorescence. Thus, a similar arrangement of the chromophores in 13,15 and 16 seems to be induced by their interactions for sufficiently flexible ring chains. The solubility of $\mathbf{1 5}$ and $\mathbf{1 6}$ is still comparably low and the side chains of the compounds slowly degrade in solution by the contact with air. As a consequence, further investigations were focused on the well-accessible compounds 18, 20, and 22 where four geminal alkyl groups are more efficiently solubilising. The UV/Vis absorption spectra of 18, 20 and 22 are similar to 2 and all are hypsochromically shifted compared with 1; Figure 5, right spectrum. The shapes are typical of perylenes in $H$-arrangements such as the cyclophanes 2 and 13 and indicate the domination of this component for light absorption. The comparably strong fluorescence of 18,20 and 22 are attributed to the $J$ component of the skew arrangement 


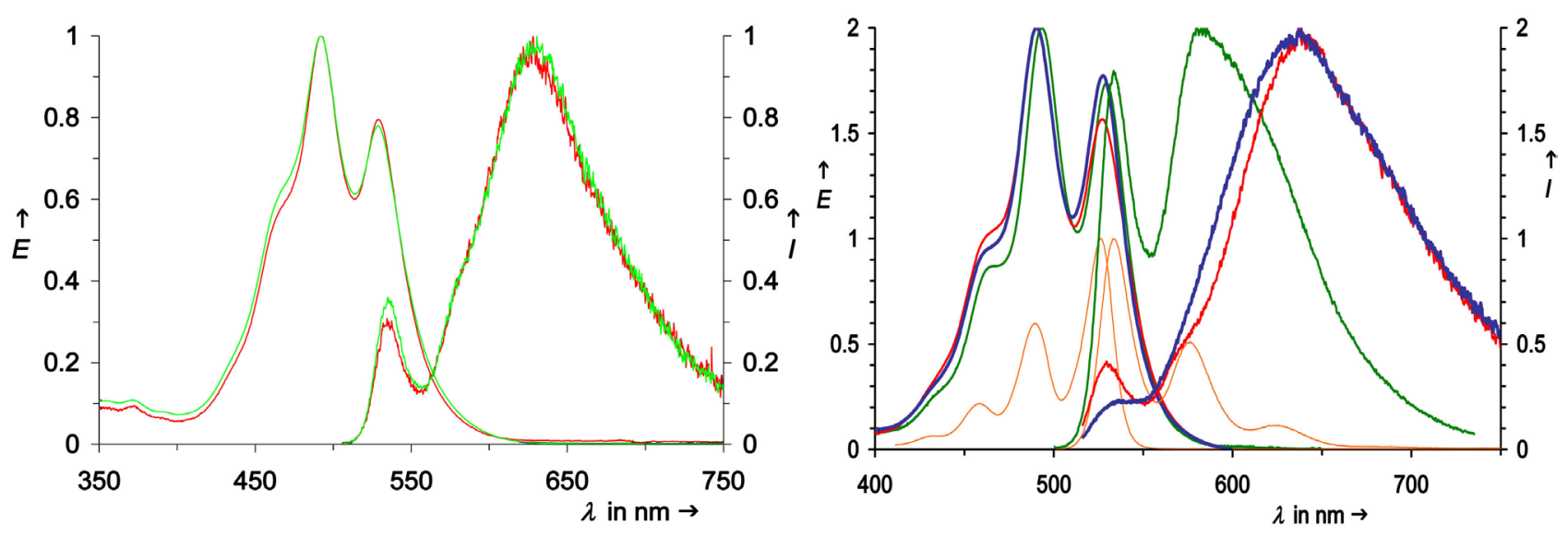

Figure 5. Left spectrum: UV/Vis absorption (left) and fluorescence (right) spectra in chloroform. Red curves: 15, green curves: 16. Right spectrum: UV/Vis absorption (left) and fluorescence (right) spectra in chloroform, $E$ and $I$ normalised to 2. Red curves: 18, green curves: 20, blue curves: 22 and yellow curves normalised to 1: 1 for comparison.

of the chromophores; this is allowed according to Förster's analysis, whereas the $H$ transition is suppressed because of symmetry. As a consequence, the $H$ transition dominates concerning light absorption and the energy of excitation is intramolecularly transferred to the $J$ transition for emission resulting in an increased Stokes' shift; the latter exhibits an alternating behaviour with the lengths of the chromophores-connecting chains. A very large Stokes' shift is obtained for 18 with a fluorescence maximum at $637.0 \mathrm{~nm}$, a medium large shift for 20 at $582.8 \mathrm{~nm}$ and again a very large shift for 22 at $638.3 \mathrm{~nm}$. Thus, two different main types of stable arrangements of chromophores seem to be reached for 18 and 22 and some other for 20. Further discussions are concentrated to the more readily soluble cyclophanes 20 and $\mathbf{2 2}$ because of the still comparably low solubility of 18. The high fluorescence quantum yield of 20 and 22 both of nearly $70 \%$ in combination with larges Stokes' shifts make these dyads of interest for fluorescence applications where the position of the fluorescence maximum can be controlled by the chain lengths of the connection of the two chromophores. Further stabilisation of the peryleneocyclophanes is possible by catalytic hydrogenation.

\section{Conclusions}

Cyclophanes of perylenebiscarboximides (perylene dyes), firstly described [10] with linear alky spacers, exhibit an increasing interest concerning redox properties [27] [28], photoswitches [29], guest host detectors and triplet generators [30] and detectors for aromatics [31] where comparably complicated starting materials and the necessary dilution principle for preparation [32] [33] mean obstacles for synthesis, upscaling and applications. The here described efficient and sustainable preparation of perylenocyclophanes in particular 20 and 22 from versatile terminal olefinic nitriles, alkylation and reduction with bulk reagents, condensation with the mass product perylene tetracarbocylic bisanhydride and subsequent ring-closing metathesis allows not only efficient synthesis but also 
technical upscaling so that applications as fluorescent dyes with large Stokes' shift such as for solar collectors [34] [35] [36] become feasible.

\section{Experimental}

\subsection{Spectroscopy}

IR spectra: Perkin Elmer 1420 Ratio Recording Infrared Spektrometer, FT 1000; UV/Vis spectra: Varian Cary 5000 and Bruins Omega 20; fluorescence spectra: Perkin Elmer FS 3000 (totally corrected); CD spectroscopy: Jasco J810 Spectropolarimeter, spectral bandwidth $0.5 \mathrm{~nm}$, integration time 0.5 and $1 \mathrm{~s}$, data interval $0.2 \mathrm{~nm}$; NMR spectroscopy: Varian Vnmrs $600(600 \mathrm{MHz})$; mass spectrometry: Finnigan MAT 95. Crystal structures: The data for kn095 and kn237 have been collected at $200 \mathrm{~K}$ on a Nonius Kappa CCD equipped with a graded multilayered X-ray optics mirror (MoKa radiation). The structures have been solved with SHELXS and refined with SHELXL [36]. The hydrogen atoms have been added in ideal geometry riding on their parent atoms. In kn237, the disorder in a side chain has been described by a split model. All split atoms have been refined isotropically. The ratio of the site occupation factors refined to $0.69 / 0.31$. The figure shows the main part only. The data have been deposited with the CCDC and can be obtained free of charge via

https://www.ccdc.cam.ac.uk/structures/: kn095 CCDC 1900229 (2,9-bis-(2,2dibutylhept-6-enyl)anthra[2,1,9-def6,5,10-d'e'f] diisoquinline-1,3,8,10-tetraone), kn237 CCDC 1900230 (2-allyl-9-(2,2-dibutylhept-6-enyl)anthra[2,1,9-def; 6,5,10-d'e'f]-diisoquinoline-1,3,8,10-tetraone). Figures are created with ORTEP [37] at the $50 \%$ ellipsoid prabability level.

\subsection{Chemicals}

Imidazole (RN 288-32-4) and perylene-3,4:9,10-tetracarboxylic bisanhydride (RN 128-69-8) were ordered from BASF. Perylene-3,4,9,10-tetracarboxylic-3, 4-anhydride-9,10-(1-hexylheptylimide) was prepared according to the literature [38].

2,2-Dibutylhex-5-enenitrile [39]: Diisopropylamine (6.89 $\mathrm{mL}, 9.48 \mathrm{mmol}$ ) in anhydrous THF $(45 \mathrm{~mL})$ under argon atmosphere at $0^{\circ} \mathrm{C}$ was subsequently treated dropwise with a solution of $2.5 \mathrm{~m} \mathrm{1-butyllithium} \mathrm{in} \mathrm{hexane}(37.9 \mathrm{~mL}$, $94.8 \mathrm{mmol}), 5$-hexenenitrile $(5.00 \mathrm{~mL}, 43.1 \mathrm{mmol})$ and 1-iodobutane $(10.8 \mathrm{~mL}$, $94.8 \mathrm{mmol})$ in anhydrous THF (40 mL, tetrahydrofurane), stirred at $0^{\circ} \mathrm{C}$ for $2 \mathrm{~h}$, allowed to warm at room temperature, stirred for $16 \mathrm{~h}$, cautiously hydrolysed (100 $\mathrm{mL}$ distilled water), extracted with diethylether, washed with distilled water $(100 \mathrm{~mL})$, saturated brine $(50 \mathrm{~mL})$ and $2 \mathrm{~N}$ aqueous $\mathrm{HCl}(50 \mathrm{~mL})$, dried with magnesiumsulphate and distilled. Yield $5.25 \mathrm{~g}(59 \%)$, b.p. $71^{\circ} \mathrm{C} / 1.6 \cdot 10^{-2} \mathrm{mbar}$, $n_{D}^{20}=1.376$. IR $(\mathrm{KBr}): \tilde{v}=3079.9 \mathrm{w}, 2957.9 \mathrm{vs}, 2937.4 \mathrm{vs}, 2864.3 \mathrm{vs}, 2230.9 \mathrm{w}$, $1833.0 \mathrm{w}, 1728.7 \mathrm{w}, 1642.4 \mathrm{~m}, 1467.8 \mathrm{~m}, 1458.4 \mathrm{~m}, 1417.4 \mathrm{w}, 1381.1 \mathrm{w}, 1342.9 \mathrm{w}$, $1302.3 \mathrm{w}, 1265.2 \mathrm{w}, 1159.0 \mathrm{w}, 1106.3 \mathrm{w}, 993.3 \mathrm{w}, 913.0 \mathrm{~m}, 731.9 \mathrm{w}, 646.2 \mathrm{w}, 557.1$ $\mathrm{cm}^{-1}$ w. ${ }^{1} \mathrm{H}$ NMR: $\left(300 \mathrm{MHz}, \mathrm{CDCl}_{3}\right): \delta=0.86\left(t, 1 \mathrm{H},{ }^{3} J=7.1 \mathrm{~Hz}\right), 1.29(\mathrm{~m}, 6 \mathrm{H}$, 
$\left.6 \mathrm{CH}_{2}\right), 1.52\left(\mathrm{~m}, 8 \mathrm{H}, 4 \mathrm{CH}_{2}\right), 2.08\left(\mathrm{~m}, 2 \mathrm{H}, \mathrm{CH}_{2}\right), 4.91\left(\mathrm{~m}, 1 \mathrm{H}, \mathrm{R}=\mathrm{CH}_{2}\right), 4.98$ $\left(\mathrm{m}, 1 \mathrm{H}, \mathrm{R}=\mathrm{CH}_{2}\right), 5.72 \mathrm{ppm}(\mathrm{m}, 1 \mathrm{H}, \mathrm{CH}) \cdot{ }^{13} \mathrm{C} \mathrm{NMR}:\left(75 \mathrm{MHz}, \mathrm{CDCl}_{3}\right): \delta=$ 13.5, 22.5, 28.3, 35.0, 35.4, 40.0 ( $\left.\mathrm{C}_{\text {quart. }}\right), 114.9$ ( $\mathrm{CH}_{2}$ olefin.), $123.3(\mathrm{CN}), 136.8$ ppm $\left(\mathrm{CH}_{2}\right.$ olefin.). MS: (DEI $\left./ 70 \mathrm{eV}\right): \mathrm{m} / z(\%): 208(5)\left[M^{+}+\mathrm{H}\right], 153(24)\left[M^{+-}\right.$ $\left.\mathrm{C}_{4} \mathrm{H}_{7}\right], 151(28)\left[M^{+}-\mathrm{C}_{4} \mathrm{H}_{9}\right], 110(100)\left[M^{+}-\mathrm{C}_{3} \mathrm{H}_{5}-\mathrm{C}_{4} \mathrm{H}_{9}\right], 97(67)\left[M^{+}-\mathrm{C}_{4} \mathrm{H}_{9}\right.$ $-\mathrm{C}_{4} \mathrm{H}_{7}$.

2-But-3-enyl-2-hexyloctanenitrile: Diisopropylamine $(2.6 \mathrm{~mL}, 90 \mathrm{mmol})$ in anhydrous THF $(40 \mathrm{~mL})$ was allowed to react with a solution of 1-butyllithium (36.0 mL, $90.0 \mathrm{mmol}, 2.5 \mathrm{~m})$, 5-hexenenitrile (4.70 mL, $40.9 \mathrm{mmol})$ and 1-iodohexane (13.3 mL, $90.0 \mathrm{mmol})$ in anhydrous THF (40 mL) according to 2,2-dibutylhex-5enenitrile. Yield $7.94 \mathrm{~g}(73 \%)$ colorless oil, b.p. $100^{\circ} \mathrm{C}-102^{\circ} \mathrm{C} / 1 \times 10^{-3} \mathrm{mbar}, n_{D}^{20}$ $=1.454$. IR $(\mathrm{KBr}): \tilde{v}=3079.6 \mathrm{w}, 2931.2 \mathrm{vs}, 2859.9 \mathrm{vs}, 2230.6 \mathrm{w}, 1826.5 \mathrm{w}$, $1730.3 \mathrm{w}, 1642.3 \mathrm{~m}, 1459.0 \mathrm{~m}, 1416.7 \mathrm{w}, 1379.0 \mathrm{w}, 1303.8 \mathrm{w}, 1078.1 \mathrm{w}, 993.6 \mathrm{w}$, $912.9 \mathrm{~m}, 759.9 \mathrm{w}, 724.6 \mathrm{w}, 648.0 \mathrm{w}, 556.0 \mathrm{~cm}^{-1} \mathrm{w} .{ }^{1} \mathrm{H} \mathrm{NMR}\left(300 \mathrm{MHz} \mathrm{CDCl}_{3}\right.$, $\left.25^{\circ} \mathrm{C}\right): \delta=0.89\left(\mathrm{t}, 6 \mathrm{H},{ }^{3} J_{\mathrm{H}, \mathrm{H}}=6.8 \mathrm{~Hz}, 2 \mathrm{CH}_{3}\right), 1.29-1.41\left(\mathrm{~m}, 16 \mathrm{H}, 8 \mathrm{CH}_{2}\right), 1.52-$

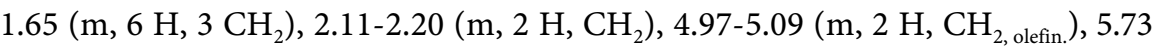
- $5.84 \mathrm{ppm}\left(\mathrm{m}, 1 \mathrm{H}, \mathrm{CH}_{\text {olefin. }}\right) .{ }^{13} \mathrm{C} \mathrm{NMR}\left(75 \mathrm{MHz}, \mathrm{CDCl}_{3}, 25^{\circ} \mathrm{C}\right): \delta=14.0,22.5$, 24.2, 28.7, 29.3, 31.5, 35.3, 36.1, $40.4\left(\mathrm{C}_{\text {quart. }}\right), 115.3\left(\mathrm{CH}_{2 \text {, olefin. }}\right), 124.0(\mathrm{CN}), 137.6$ ppm $\left(\mathrm{CH}_{\text {olefin. }}\right)$. MS $\left(\mathrm{DEI}^{+} / 70 \mathrm{eV}\right) \mathrm{m} / z(\%): 264(4)\left[M^{+}+\mathrm{H}\right], 263(4)\left[M^{+}\right], 262$ (5) $\left[M^{+}-\mathrm{H}\right], 206(22)\left[M^{+}-\mathrm{C}_{4} \mathrm{H}_{9}\right], 192(21)\left[M^{+}-\mathrm{C}_{5} \mathrm{H}_{11}\right], 179$ (43) $\left[M^{+}-\right.$ $\left.\mathrm{C}_{6} \mathrm{H}_{13}\right], 138(100)\left[M^{+}-\mathrm{C}_{6} \mathrm{H}_{13}-\mathrm{C}_{3} \mathrm{H}_{5}\right], 125(35)\left[M^{+}-\mathrm{C}_{6} \mathrm{H}_{13}-\mathrm{C}_{4} \mathrm{H}_{7}\right], 97$ (26) $\left[M^{+}-2 \times \mathrm{C}_{6} \mathrm{H}_{13}\right], 55(17)\left[\mathrm{C}_{4} \mathrm{H}_{9}\right]$.

2-But-3-enyl-2-octyldecanenitrile: Diisopropylamine $(12.7 \mathrm{~mL} 90.2 \mathrm{mmol})$ in anhydrous THF (45 mL), a solution of 1-butyllithium $(36.1 \mathrm{~mL}, 90.2 \mathrm{mmol}$, $2.5 \mathrm{~m})$; 5-hexenenitrile $(4.76 \mathrm{ml}, 41.0 \mathrm{mmol})$ and 1-iodoctane $(22.3 \mathrm{ml}, 133$ mmol; 3 eq.) in anhydrous THF $(50 \mathrm{~mL})$ were allowed to react according to 2,2-dibutylhex-5-enenitrile. Yield $10.7 \mathrm{~g}$ (82\%) light yelowish, viscous oil, b.p. $140^{\circ} \mathrm{C}-144^{\circ} \mathrm{C} / 1 \times 10^{-3} \mathrm{mbar}, n_{D}^{20}=1.457 .-\mathrm{IR}(\mathrm{KBr}): \tilde{v}=3079.7 \mathrm{w}, 2926.9 \mathrm{vs}$, 2856.0 s, 2230.9 w, 1731.3 w, 1642.5 w, 1465.6 m, 1378.3 w, 1157.5 w, 1071.5 w, $992.3 \mathrm{w}, 912.6 \mathrm{w}, 711.4 \mathrm{~cm}^{-1}$ w. ${ }^{1} \mathrm{H}$ NMR (300 $\left.\mathrm{MHz} \mathrm{CDCl}_{3}, 25^{\circ} \mathrm{C}\right): \delta=0.89(\mathrm{t}$, $\left.{ }^{3} \mathrm{~J}_{\mathrm{H}, \mathrm{H}}=6.9 \mathrm{~Hz}, 6 \mathrm{H}, 2 \mathrm{CH}_{3}\right), 1.28-1.31\left(\mathrm{~m}, 20 \mathrm{H}, 10 \mathrm{CH}_{2}\right), 1.40-1.41(\mathrm{~m}, 4 \mathrm{H}, 2$ $\left.\mathrm{CH}_{2}\right), 1.54-1.58\left(\mathrm{~m}, 4 \mathrm{H}, 2 \mathrm{CH}_{2}\right), 1.62-1.65\left(\mathrm{~m}, 2 \mathrm{H}, \mathrm{CH}_{2}\right), 2.15-2.18(\mathrm{~m}, 2 \mathrm{H}$, $\mathrm{CH}_{2}$ ), 4.99 - 5.01 (m, $2 \mathrm{H}, \mathrm{CH}_{2 \text {, olefin. }}$ ), 5.77 - $5.84 \mathrm{ppm}\left(\mathrm{m}, 1 \mathrm{H}, \mathrm{CH}_{\text {olefin. }}\right) .{ }^{13} \mathrm{C} \mathrm{NMR}$ $\left(75 \mathrm{MHz}, \mathrm{CDCl}_{3}, 25^{\circ} \mathrm{C}\right): \delta=14.0,22.5,24.1,29.1,29.3,29.6,31.7,35.3,36.0,40.4$ $\left(\mathrm{C}_{\text {quart. }}\right), 115.2\left(\mathrm{CH}_{2, \text { olefin. }}\right), 123.9(\mathrm{CN}), 137.1 \mathrm{ppm}\left(\mathrm{CH}_{\text {olefin. }}\right) . \mathrm{MS}\left(\mathrm{DEI}^{+} / 70 \mathrm{eV}\right)$ $m / z(\%): 318$ (4) $\left[M^{+}-\mathrm{H}\right], 207$ (46) $\left[M^{+-} \mathrm{C}_{8} \mathrm{H}_{17}\right], 166(100)\left[M^{+}-\mathrm{C}_{8} \mathrm{H}_{17}-\right.$ $\left.\mathrm{C}_{3} \mathrm{H}_{5}\right], 95(15)\left[M^{+}-2 \times \mathrm{C}_{8} \mathrm{H}_{17}\right], 55(60)\left[M^{+}-2 \times \mathrm{C}_{8} \mathrm{H}_{17}-\mathrm{C}_{3} \mathrm{H}_{5}\right]$.

2-Butyl-2-vinylhexanenitrile: Diisopropylamine $(11.5 \mathrm{~mL}, 82.1 \mathrm{mmol})$ in anhydrous THF (40 mL), a solution of 1-butyllithium $(32.8 \mathrm{~mL}, 82.1 \mathrm{mmol}$, $2.5 \mathrm{~m})$, allylcyanide $(2.50 \mathrm{~g}, 37.3 \mathrm{mmol})$ and 1 -iodobutane $(9.34 \mathrm{~mL}, 82.1$ $\mathrm{mmol})$ in anhydrous THF $(45 \mathrm{~mL})$ were allowed to react according to 2,2-dibutylhex-5-enenitrile. Yield $1.54 \mathrm{~g}(23 \%)$ colorless liquid, b.p. $43^{\circ} \mathrm{C} / 8 \times 10^{-3}$ mbar, $n_{D}^{20}=1.441$. IR $(\mathrm{KBr}): \tilde{v}=3088.0 \mathrm{w}, 2959.3 \mathrm{vs}, 2936.9$ vs, 2864.4 vs, 
2237.2 w, $1853.1 \mathrm{w}, 1736.3 \mathrm{w}, 1639.5 \mathrm{w}, 1468.0 \mathrm{~m}, 1411.5 \mathrm{w}, 1381.0 \mathrm{w}, 1343.3 \mathrm{w}$, $1302.5 \mathrm{w}, 1260.7 \mathrm{w}, 1158.5 \mathrm{w}, 1111.7 \mathrm{w}, 1066.6 \mathrm{w}, 989.5 \mathrm{~m}, 925.1 \mathrm{~m}, 785.1 \mathrm{w}$, $753.2 \mathrm{w}, 731.2 \mathrm{w}, 695.8 \mathrm{w}, 587.5 \mathrm{~cm}^{-1}$ w. ${ }^{1} \mathrm{H}$ NMR $\left(300 \mathrm{MHz}, \mathrm{CDCl}_{3}, 25^{\circ} \mathrm{C}\right): \delta=$ $0.89\left(\mathrm{t},{ }^{3} J_{\mathrm{H}, \mathrm{H}}=7.0 \mathrm{~Hz}, 6 \mathrm{H}, 2 \mathrm{CH}_{3}\right), 1.24-1.52\left(\mathrm{~m}, 10 \mathrm{H}, 5 \mathrm{CH}_{2}\right), 1.63-1.73(\mathrm{~m}, 2$ $\left.\mathrm{H}, \mathrm{CH}_{2}\right), 5.22-5.25\left(\mathrm{~m}, 2 \mathrm{H}, \mathrm{CH}_{2 \text {, olefin. }}\right), 5.42-5.44 \mathrm{ppm}\left(\mathrm{m}, 1 \mathrm{H}, \mathrm{CH}_{\text {olefin. }}\right) \cdot{ }^{13} \mathrm{C}$ $\operatorname{NMR}\left(75 \mathrm{MHz}, \mathrm{CDCl}_{3}, 25^{\circ} \mathrm{C}\right): \delta=13.8,22.6,27.0,38.6,46.1\left(\mathrm{C}_{\text {quart. }}\right), 116.5\left(\mathrm{CH}_{2}\right.$, olefin.), $121.5(\mathrm{CN}), 137.2 \mathrm{ppm}\left(\mathrm{CH}_{\text {olefin. }}\right)$. MS (DEI $\left./ 70 \mathrm{eV}\right) \mathrm{m} / z(\%): 180(4)\left[M^{+}+\right.$ $\mathrm{H}], 153(4)\left[M^{+}-\mathrm{C}_{2} \mathrm{H}_{3}\right], 136(5)\left[M^{+}-\mathrm{C}_{3} \mathrm{H}_{7}\right], 123(100)\left[M^{+}-\mathrm{C}_{4} \mathrm{H}_{9}\right], 108$ (37) $\left[M^{+}-\mathrm{C}_{3} \mathrm{H}_{7}-\mathrm{C}_{2} \mathrm{H}_{3}\right], 94(22)\left[M^{+}-\mathrm{C}_{4} \mathrm{H}_{9}-\mathrm{C}_{2} \mathrm{H}_{3}\right], 80(50)\left[M^{+}-\mathrm{C}_{4} \mathrm{H}_{9}-\mathrm{C}_{3} \mathrm{H}_{7}\right]$.

2-Allyl-2-butylhexanenitrile: Diisopropylamine $(15.3 \mathrm{~mL}, 109 \mathrm{mmol})$ in anhydrous THF (50 mL), a solution of 1-butyllithium $(43.6 \mathrm{~mL}, 109 \mathrm{mmol}, 2.5$ $\mathrm{m}), 4$-pentenenitrile $(4.90 \mathrm{~g}, 49.6 \mathrm{mmol})$ and 1-iodobutane $(12.4 \mathrm{~mL}, 109 \mathrm{mmol})$ in anhydrous THF (45 mL) were allowed to react according to 2,2-dibutylhex-5enenitrile. Yield $8.31 \mathrm{~g}(87 \%)$ colorless liquid, b.p. $62^{\circ} \mathrm{C}-64^{\circ} \mathrm{C} / 1 \times 10^{-2} \mathrm{mbar}$, $n_{D}^{20}=1.447 . \mathrm{IR}(\mathrm{KBr}): \tilde{v}=3081.0 \mathrm{w}, 2958.8 \mathrm{vs}, 2937 \mathrm{vs}, 2864.5 \mathrm{~s}, 2231.9 \mathrm{w}$, $1737.5 \mathrm{w}, 1642.6 \mathrm{w}, 1467.9 \mathrm{~m}, 1417.8 \mathrm{w}, 1381.6 \mathrm{w}, 1342.7 \mathrm{w}, 994.9 \mathrm{~m}, 920.8 \mathrm{~m}$, $732.2 \mathrm{w}, 565.1 \mathrm{~cm}^{-1}$ w. ${ }^{1} \mathrm{H}$ NMR $\left(300 \mathrm{MHz}, \mathrm{CDCl}_{3}, 25^{\circ} \mathrm{C}\right): \delta=0.92\left(\mathrm{t},{ }^{3} J=7.1 \mathrm{~Hz}\right.$, $\left.6 \mathrm{H}, 2 \mathrm{CH}_{3}\right), 1.28-1.46\left(\mathrm{~m}, 8 \mathrm{H}, 4 \mathrm{CH}_{2}\right), 1.51-1.59\left(\mathrm{~m}, 4 \mathrm{H}, 2 \mathrm{CH}_{2}\right), 2.31-2.34$ (m, $\left.2 \mathrm{H}, \mathrm{CH}_{2}\right), 5.13$ - $5.21\left(\mathrm{~m}, 2 \mathrm{H}, \mathrm{CH}_{2 \text {, olefin. }}\right), 5.73-5.87 \mathrm{ppm}\left(\mathrm{m}, 1 \mathrm{H}, \mathrm{CH}_{\text {olefin. }}\right)$. ${ }^{13} \mathrm{C}$ NMR $\left(75 \mathrm{MHz}, \mathrm{CDCl}_{3}, 25^{\circ} \mathrm{C}\right): \delta=13.9,22.8,26.4,35.7,40.3,40.4\left(\mathrm{C}_{\text {quart }}\right)$, $119.6\left(\mathrm{CH}_{2, \text { olefin. }}\right), 123.8(\mathrm{CN}), 131.9 \mathrm{ppm}\left(\mathrm{CH}_{\text {olefin. }}\right)$. - MS $\left(\mathrm{DEI}^{+} / 70 \mathrm{eV}\right) \mathrm{m} / z(\%)$ : $194(4)\left[M^{+}+\mathrm{H}\right], 150(100)\left[M^{+}-\mathrm{C}_{3} \mathrm{H}_{7}\right], 137(17)\left[M^{+}-\mathrm{C}_{4} \mathrm{H}_{9}\right], 110(22)\left[M^{+}-\right.$ $\left.\mathrm{C}_{3} \mathrm{H}_{7}-\mathrm{C}_{3} \mathrm{H}_{5}\right], 55(11)\left[M^{+}-\mathrm{C}_{4} \mathrm{H}_{9}-\mathrm{C}_{3} \mathrm{H}_{7}-\mathrm{C}_{3} \mathrm{H}_{5}\right], 40(33)\left[M^{+}-2 \times \mathrm{C}_{4} \mathrm{H}_{9}-\right.$ $\mathrm{C}_{3} \mathrm{H}_{5}$.

2,2-Dibutylhept-6-enenitrile: Diisopropylamine (14.1 $\mathrm{mL}, 101 \mathrm{mmol}$ ) in anhydrous THF (45 mL), a solution of 1-butyllithium (40.4 mL, $101 \mathrm{mmol}, 2.5$ $\mathrm{m})$, 6-heptenenitrile $(5.00 \mathrm{~g}, 45.8 \mathrm{mmol})$ and 1-iodobutane $(11.4 \mathrm{~mL}, 101 \mathrm{mmol})$ in anhydrous THF $(30 \mathrm{~mL})$ were allowed to react according to 2,2-dibutylhex-5enenitrile. Yield $9.04 \mathrm{~g}(89 \%)$ colorless oil, b.p. $85^{\circ} \mathrm{C} / 1 \cdot 10^{-3} \mathrm{mbar}, n_{D}^{20}=1.451$. IR (KBr): $\tilde{v}=3080.3 \mathrm{w}, 2957.2$ vs, 2929.9 vs, 2872.0 s, 2860.5 s, $2230.6 \mathrm{w}$, $1666.3 \mathrm{~m}, 1636.9 \mathrm{~m}, 1535.0 \mathrm{w}, 1466.5 \mathrm{~m}, 1414.1 \mathrm{w}, 1378.3 \mathrm{w}, 1260.9 \mathrm{w}, 1235.9 \mathrm{w}$, $1155.8 \mathrm{w}, 1095.9 \mathrm{w}, 1003.9 \mathrm{w}, 910.5 \mathrm{~m}, 802.1 \mathrm{w}, 729.3 \mathrm{~cm}^{-1}$ w. ${ }^{1} \mathrm{H}$ NMR (300 $\left.\mathrm{MHz}, \mathrm{CDCl}_{3}, 25^{\circ} \mathrm{C}\right): \delta=0.92\left(\mathrm{t},{ }^{3} J=7.0 \mathrm{~Hz}, 6 \mathrm{H}, 2 \mathrm{CH}_{3}\right), 1.29-1.39(\mathrm{~m}, 8 \mathrm{H}, 4$ $\left.\mathrm{CH}_{2}\right), 1.48-1.55\left(\mathrm{~m}, 8 \mathrm{H}, 4 \mathrm{CH}_{2}\right), 2.07\left(\mathrm{q},{ }^{3} \mathrm{~J}=6.5 \mathrm{~Hz}, 2 \mathrm{H}, \mathrm{CH}_{2}\right), 4.95-5.05(\mathrm{~m}$, $\left.2 \mathrm{H}, \mathrm{CH}_{2 \text {, olefin. }}\right), 5.71-5.84 \mathrm{ppm}\left(\mathrm{m}, 1 \mathrm{H}, \mathrm{CH}_{\text {olefin. }}\right) .{ }^{13} \mathrm{C} \mathrm{NMR}\left(75 \mathrm{MHz} \mathrm{CDCl}_{3}\right.$, $\left.25^{\circ} \mathrm{C}\right): \delta=13.8,22.8,23.5,26.3,33.6,35.5,35.8,40.4\left(\mathrm{C}_{\text {quart. }}\right), 115.1\left(\mathrm{CH}_{2 \text {, olefin. }}\right)$, $124.2(\mathrm{CN}), 137.8 \mathrm{ppm}\left(\mathrm{CH}_{\text {olefin. }}\right)$. MS (DEI $\left./ 70 \mathrm{eV}\right) \mathrm{m} / z(\%): 222(1)\left[M^{+}-\mathrm{H}\right]$, $165(33)\left[M^{+}-\mathrm{C}_{4} \mathrm{H}_{9}\right], 153(4)\left(M^{+}-\mathrm{C}_{5} \mathrm{H}_{9}\right], 122(100)\left[M^{+}-\mathrm{C}_{4} \mathrm{H}_{9}-\mathrm{C}_{3} \mathrm{H}_{7}\right], 110$ $\left(M^{+}-\mathrm{C}_{5} \mathrm{H}_{9}-\mathrm{C}_{3} \mathrm{H}_{7}\right], 55(9)\left[M^{+}-2 \times \mathrm{C}_{4} \mathrm{H}_{9}-\mathrm{C}_{4} \mathrm{H}_{7}\right], 41(18)\left[M^{+}-2 \times \mathrm{C}_{4} \mathrm{H}_{9}-\right.$ $\left.\mathrm{C}_{5} \mathrm{H}_{9}\right]$.

2,2-Diisopropylbut-3-enenitrile (method 1): Diisopropylamine (23.1 $\mathrm{mL}$, $164 \mathrm{mmol})$ in anhydrous THF $(50 \mathrm{~mL})$, a solution of 1-butyllithium $(65.6 \mathrm{~mL}$, $164 \mathrm{mmol}, 2.5 \mathrm{~m})$, allylcyanide $(5.00 \mathrm{~g}, 74.5 \mathrm{mmol})$ und 2-iodopropane $(6.40$ $\mathrm{mL}, 164 \mathrm{mmol})$ in anhydrous THF $(50 \mathrm{~mL})$ were allowed to react according to 
2,2-dibutylhex-5-enenitrile. Yield $940 \mathrm{mg}$ (8\%) colorless liquid, b.p. $25^{\circ} \mathrm{C}-26^{\circ} \mathrm{C} / 1$ $\times 10^{-3}$ mbar (for the further characterisation see method 2).

2,2-Diisopropylbut-3-enenitrile (method 2): Lithiumhexamethyldisilazane $\left(100 \mathrm{~mL}, 0.1 \mathrm{~m}\right.$ solution) under argon atmosphere was cooled at $0^{\circ} \mathrm{C}$ and treated dropwise with stirring within $20 \mathrm{~min}$ with a solution of allylcyanide $(3.05 \mathrm{~g}, 45.5$ $\mathrm{mmol})$ in anhydrous THF $(15 \mathrm{~mL})$ and then with 2-iodopropane $(17.0 \mathrm{~g}, 100$ $\mathrm{mmol}$ ), stirred at $0^{\circ} \mathrm{C}$ for $90 \mathrm{~min}$, allowed to warm slowly at room temperature, stirred for $16 \mathrm{~h}$, cautiously hydrolised with distilled water $(50 \mathrm{~mL})$, treated drop wise with $2 \mathrm{~N}$ aqueous $\mathrm{HCl}$ (100 $\mathrm{mL}$, strong evolution of foam), extracted with diethylether $(3 \times 100 \mathrm{~mL})$, dried with magnesiumsulphate, evaporated and distilled. Yield $1.18 \mathrm{~g}(10 \%)$ colorless liquid, b.p. $62-63^{\circ} \mathrm{C} / 20 \mathrm{mbar}, n_{D}^{20}=1.447$. IR $(\mathrm{KBr}): \tilde{v}=3087.1 \mathrm{w}, 2969.3 \mathrm{vs}, 2938.0 \mathrm{~m}, 2878.8 \mathrm{~m}, 2234.6 \mathrm{w}, 1639.9 \mathrm{w}$, $1466.8 \mathrm{~m}, 1412.9$ w, $1390.9 \mathrm{~m}, 1375.4 \mathrm{w}, 1315.7 \mathrm{w}, 1260.2 \mathrm{w}, 1203.7 \mathrm{w}, 1171.6 \mathrm{w}$, $1063.5 \mathrm{w}, 994.2 \mathrm{~m}, 925.5 \mathrm{~m}, 803.0 \mathrm{w}, 699.0,672.0 \mathrm{~cm}^{-1} .{ }^{1} \mathrm{H}$ NMR $(300 \mathrm{MHz}$, $\left.\mathrm{CDCl}_{3}, 25^{\circ} \mathrm{C}\right): \delta=0.93\left(\mathrm{~d},{ }^{3} J=6.8 \mathrm{~Hz}, 6 \mathrm{H}, 2 \mathrm{CH}_{3}\right), 1.05\left(\mathrm{~d},{ }^{3} J=6.7 \mathrm{~Hz}, 6 \mathrm{H}, 2\right.$ $\mathrm{CH}_{3}$ ), 2.03 (sept., ${ }^{3} \mathrm{~J}=6.8 \mathrm{~Hz}, 2 \mathrm{H}, 2 \mathrm{CH}_{\text {aliphat. }}$ ), $5.33-5.39$ (m, $1 \mathrm{H}, \mathrm{CH}_{\text {olefin. }}$ ), 5.44 - $5.47 \mathrm{ppm}\left(\mathrm{m}, 2 \mathrm{H}, \mathrm{CH}_{2}\right.$, olefin. $){ }^{13} \mathrm{C} \mathrm{NMR}\left(75 \mathrm{MHz}, \mathrm{CDCl}_{3}, 25^{\circ} \mathrm{C}\right): \delta=17.3,18.4$, 31.6, $55.7\left(\mathrm{C}_{\text {quart. }}\right), 118.7\left(\mathrm{CH}_{2}\right.$, olefin. $), 120.4(\mathrm{CN}), 132.6 \mathrm{ppm}\left(\mathrm{CH}_{\text {olefin. }}\right)$. MS $\left(\mathrm{DEI}^{+} / 70 \mathrm{eV}\right) \mathrm{m} / z(\%): 152(0.2)\left[M^{+}+\mathrm{H}\right], 109(64)\left[M^{+}-\mathrm{C}_{3} \mathrm{H}_{7}\right], 94\left[M^{+}-\mathrm{C}_{3} \mathrm{H}_{7}\right.$ $\left.-\mathrm{CH}_{3}\right], 67(10)\left[M^{+}-2 \times \mathrm{C}_{3} \mathrm{H}_{7}\right], 41(13)\left[M^{+}-2 \times \mathrm{C}_{3} \mathrm{H}_{7}-\mathrm{C}_{2} \mathrm{H}_{3}\right]$.

2,2-Diisopropylhex-5-enenitrile: Diisopropylamine (12.7 mL, $90.2 \mathrm{mmol})$ in anhydrous THF ( $45 \mathrm{~mL})$, a solution of 1-butyllithium (36.1 mL, $90.2 \mathrm{mmol}, 2.5 \mathrm{~m})$, 5-hexenenitrile (3.90 g, $41.0 \mathrm{mmol}$ ) and 2-iodopropane (9.00 mL, $90.2 \mathrm{mmol})$ in anhydrous THF $(40 \mathrm{~mL})$ were allowed to react according to 2,2-dibutylhex-5enenitrile. Yield $4.51 \mathrm{~g}(62 \%)$ colorless liquid, b.p. $56^{\circ} \mathrm{C}-58^{\circ} \mathrm{C} / 1 \times 10^{-3} \mathrm{mbar}$, $n_{D}^{20}=1.455 . \mathrm{IR}(\mathrm{KBr}): \tilde{v}=3079.7 \mathrm{w}, 2971.8 \mathrm{vs}, 2880.5 \mathrm{~m}, 2228.7 \mathrm{w}, 1736.2 \mathrm{w}$, $1642.5 \mathrm{~m}, 1471.0 \mathrm{~m}, 1417.6 \mathrm{w}, 1392.3 \mathrm{~m}, 1375.9 \mathrm{w}, 1309.5 \mathrm{w}, 1175.0 \mathrm{w}, 1130.7 \mathrm{w}$, 992.9 w, $913.7 \mathrm{~m}, 757.9$ w, 698.0 w, $617.4 \mathrm{~cm}^{-1}$ w. ${ }^{1} \mathrm{H} \mathrm{NMR}\left(300 \mathrm{MHz}, \mathrm{CDCl}_{3}\right.$, $\left.25^{\circ} \mathrm{C}\right): \delta=0.97-1.00\left(\mathrm{~m}, 6 \mathrm{H}, 2 \mathrm{CH}_{3}\right), 1.06-1.08\left(\mathrm{~m}, 6 \mathrm{H}, 2 \mathrm{CH}_{3}\right), 1.51-1.58$ $\left(\mathrm{m}, 2 \mathrm{H}, \mathrm{CH}_{2}\right), 1.92-2.06(\mathrm{~m}, 2 \mathrm{H}, 2 \mathrm{CH}), 2.16-2.25\left(\mathrm{~m}, 2 \mathrm{H}, \mathrm{CH}_{2}\right), 4.94-5.09$ $\left(\mathrm{m}, 2 \mathrm{H}, \mathrm{CH}_{2, \text { olefin. }}\right), 5.71-5.84 \mathrm{ppm}\left(\mathrm{m}, 1 \mathrm{H}, \mathrm{CH}_{\text {olefin. }}\right) .{ }^{13} \mathrm{C} \mathrm{NMR}(75 \mathrm{MHz}$, $\left.\mathrm{CDCl}_{3}, 25^{\circ} \mathrm{C}\right): \delta=17.5,18.8,29.5,31.7,32.6,49.1\left(\mathrm{C}_{\text {quart. }}\right), 115.0\left(\mathrm{CH}_{2, \text { olefin. }}\right), 122.2$ (CN), $137.3 \mathrm{ppm}\left(\mathrm{CH}_{\text {olefin. }}\right)$. MS $\left(\mathrm{DEI}^{+} / 70 \mathrm{eV}\right) \mathrm{m} / z(\%): 180(1)\left[M^{+}+\mathrm{H}\right], 164(3)$ $\left[M^{+}-\mathrm{CH}_{3}\right], 137(5)\left[M^{+}-\mathrm{C}_{3} \mathrm{H}_{7}\right], 122(8)\left[M^{+}-\mathrm{C}_{3} \mathrm{H}_{7}-\mathrm{CH}_{3}\right], 110(10)\left[M^{+}-\right.$ $\left.\mathrm{C}_{3} \mathrm{H}_{7}-2 \times \mathrm{CH}_{3}\right], 96(100)\left[M^{+}-2 \times \mathrm{C}_{3} \mathrm{H}_{7}\right], 55(8)\left[M^{+}-2 \times \mathrm{C}_{3} \mathrm{H}_{7}-\mathrm{C}_{3} \mathrm{H}_{5}\right], 41$ (19) $\left[M^{+}-\mathrm{C}_{4} \mathrm{H}_{7}-2 \times \mathrm{C}_{3} \mathrm{H}_{7}\right]$.

2,2-Dibutylhex-5-enylamine: Lithiumaluminiumhydride (1.54 g, $40.5 \mathrm{mmol}$ ) under nitrogen atmosphere was disperged in anhydrous diethylether $(110 \mathrm{~mL})$ and treated dropwise and stirring with 1,1-dibutylhex-5-enenitrile (5.25 g, 25.3 $\mathrm{mmol})$ in anhydrous diethylether $(20 \mathrm{~mL})$, heated under reflux for $3 \mathrm{~h}$, allowed to cool, treated dropwise with $30 \%$ aqueous $\mathrm{NaOH}(10 \mathrm{~mL})$, diluted with distilled water $(50 \mathrm{~mL})$, collected with the organic phase, extracted with brine (20 $\mathrm{mL}$ ), dried with magnesiumsulphate, evaporated and distilled in vacuo. Yield 
$3.54 \mathrm{~g}(66 \%)$ colorless liquid, b.p. $65^{\circ} \mathrm{C}-66^{\circ} \mathrm{C} / 1.2 \times 10^{-2} \mathrm{mbar}, n_{D}^{20}=1.459$. IR $(\mathrm{KBr}): \tilde{v}=3391.2 \mathrm{w}, 3306 \mathrm{w}, 3076.5 \mathrm{w}, 2956.7 \mathrm{vs}, 2929.9 \mathrm{vs}, 2861.3 \mathrm{vs}, 1819.1$ w, $1640.4 \mathrm{~m}, 1580.3 \mathrm{~m}, 1468.0 \mathrm{~m}, 1378.8 \mathrm{~m}, 1292.2 \mathrm{~m}, 1141.7 \mathrm{w}, 1067.7 \mathrm{w}, 993.7$ w, $907.8 \mathrm{~m}, 815.7 \mathrm{w}, 729.1 \mathrm{w}, 636.2 \mathrm{w}, 557.2 \mathrm{~cm}^{-1}$ w. ${ }^{1} \mathrm{H} \mathrm{NMR}\left(600 \mathrm{MHz}, \mathrm{CDCl}_{3}\right.$, $\left.25^{\circ} \mathrm{C}\right): \delta=0.83\left(\mathrm{t},{ }^{3} J=7.3 \mathrm{~Hz}, 6 \mathrm{H}, 2 \mathrm{CH}_{3}\right), 1.05-1.10\left(\mathrm{~m}, 8 \mathrm{H}, 4 \mathrm{CH}_{2}\right), 1.17-$ $1.23\left(\mathrm{~m}, 6 \mathrm{H}, 2 \mathrm{CH}_{3}\right), 1.84-1.88\left(\mathrm{~m}, 2 \mathrm{H}, \mathrm{CH}_{2}\right), 2.39\left(\mathrm{~s}, 2 \mathrm{H}, \mathrm{N}-\mathrm{CH}_{2}\right), 4.83-4.94$ $\left(\mathrm{m}, 2 \mathrm{H}, \mathrm{CH}_{2, \text { olefin. }}\right), 5.70-5.77 \mathrm{ppm}\left(\mathrm{m}, 1 \mathrm{H}, \mathrm{CH}_{\text {olefin. }}\right){ }^{13} \mathrm{C} \mathrm{NMR}(150 \mathrm{MHz}$, $\left.\mathrm{CDCl}_{3}, 25^{\circ} \mathrm{C}\right): \delta=14.0,23.5,25.0,27.4,33.6,34.0,38.7,47.0\left(\mathrm{CH}_{2}-\mathrm{N}\right), 113.7$ $\left(\mathrm{CH}_{2 \text {, olefin. }}\right), 139.3 \mathrm{ppm}\left(\mathrm{CH}_{\text {olefin. }}\right) . \mathrm{MS}\left(\mathrm{DEI}^{+} / 70 \mathrm{eV}\right) \mathrm{m} / z(\%): 212(6)\left[M^{+}+\mathrm{H}\right]$, $196(42)\left[M^{+}-\mathrm{NH}_{2}\right], 154(61)\left[M^{+}-\mathrm{NH}_{2}-\mathrm{C}_{3} \mathrm{H}_{5}\right], 140(56)\left[M^{+}-\mathrm{C}_{4} \mathrm{H}_{7}-\mathrm{NH}_{2}\right]$, 97 (81) $\left[M^{+}-2 \times \mathrm{C}_{4} \mathrm{H}_{9}\right], 83(91)\left[M^{+}-2 \times \mathrm{C}_{4} \mathrm{H}_{9}-\mathrm{NH}_{2}\right], 69(100)\left[M^{+} \mathrm{C}_{4} \mathrm{H}_{7}-\right.$ $\left.\mathrm{C}_{4} \mathrm{H}_{9}-\mathrm{CH}_{2} \mathrm{NH}_{2}\right]$.

2-But-3-enyl-2-hexyloctylamine: Lithiumaluminiumhydride (1.76 g, 46.5 $\mathrm{mmol})$ in anhydrous diethylether $(110 \mathrm{~mL}), 2$-but-3-enyl-2-hexyloctanenitrile (7.90 $\mathrm{g}, 30.0 \mathrm{mmol}$ ) in anhydrous diethylether $(40 \mathrm{~mL})$ and subsequently $30 \%$ aqueous $\mathrm{NaOH}(15 \mathrm{~mL})$ were allowed to react as was described for 2,2-dibutylhex-5enylamine. Yield $5.58 \mathrm{~g}(70 \%)$ colorless oil, b.p. $96-98^{\circ} \mathrm{C} / 1 \cdot 10^{-3} \mathrm{mbar}, n_{D}^{20}=$ 1.462. IR (KBr): $\tilde{v}=3076.8 \mathrm{w}, 2956.1 \mathrm{~s}, 2928.1 \mathrm{vs,} 2858.6 \mathrm{~s}, 1725.0 \mathrm{w}, 1640.4 \mathrm{w}$, 1467.1 m, $1378.2 \mathrm{w}, 1300.7 \mathrm{w}, 1070.5 \mathrm{w}, 993.7 \mathrm{w}, 908.0 \mathrm{~m}, 815.5 \mathrm{w}, 724.1 \mathrm{w}$, $636.9 \mathrm{~cm}^{-1}$ w. ${ }^{1} \mathrm{H}$ NMR $\left(300 \mathrm{MHz}, \mathrm{CDCl}_{3}, 25^{\circ} \mathrm{C}\right): \delta=0.86\left(\mathrm{t},{ }^{3} J=6.8 \mathrm{~Hz}, 6 \mathrm{H}, 2\right.$ $\left.\mathrm{CH}_{3}\right), 1.13-1.29\left(\mathrm{~m}, 22 \mathrm{H}, 11 \mathrm{CH}_{2}\right), 1.86-1.94\left(\mathrm{~m}, 2 \mathrm{H}, \mathrm{CH}_{2}\right), 2.43(\mathrm{~s}, 2 \mathrm{H}$, $\left.\mathrm{CH}_{2}-\mathrm{N}\right), 4.87-5.01\left(\mathrm{~m}, 2 \mathrm{H}, \mathrm{CH}_{2}\right.$, olefin. $), 5.72-5.86 \mathrm{ppm}\left(\mathrm{m}, 1 \mathrm{H}, \mathrm{CH}_{\text {olefin. }}\right) .{ }^{13} \mathrm{C}$ $\operatorname{NMR}\left(75 \mathrm{MHz}, \mathrm{CDCl}_{3}, 25^{\circ} \mathrm{C}\right): \delta=14.0,22.6,22.8,27.5,30.2,31.8,33.7,34.4$, 38.9, $47.1\left(\mathrm{CH}_{2}-\mathrm{N}\right), 113.8\left(\mathrm{CH}_{2}\right.$,olefin. $), 139.5 \mathrm{ppm}\left(\mathrm{CH}_{\text {olefin. }}\right)$. MS $\left(\mathrm{DEI}^{+} / 70 \mathrm{eV}\right) \mathrm{m} / \mathrm{z}$ (\%): $266(10)\left[M^{+}-\mathrm{H}\right], 252(58)\left[M^{+}-\mathrm{NH}_{2}\right], 238(35)\left[M^{+}-\mathrm{CH}_{4} \mathrm{~N}_{1}, 196\right.$ (61) $\left[M^{+}-\mathrm{C}_{5} \mathrm{H}_{11}\right], 182(100)\left[M^{+}-\mathrm{C}_{6} \mathrm{H}_{13}\right], 151\left[M^{+}-\mathrm{C}_{6} \mathrm{H}_{13}-\mathrm{CH}_{4} \mathrm{~N}\right], 111\left[M^{+}-\right.$ $\left.\mathrm{C}_{6} \mathrm{H}_{13}-\mathrm{C}_{5} \mathrm{H}_{11}\right], 97(81)\left[M^{+}-2 \times \mathrm{C}_{6} \mathrm{H}_{13}\right], 83(79)\left[M^{+-} 2 \times \mathrm{C}_{6} \mathrm{H}_{13}-\mathrm{NH}_{2}\right], 69$ (71) $\left[M^{+}-2 \times \mathrm{C}_{6} \mathrm{H}_{13}-\mathrm{CH}_{4} \mathrm{~N}\right], 55(52)\left[M^{+}-2 \times \mathrm{C}_{6} \mathrm{H}_{13}-\mathrm{C}_{2} \mathrm{H}_{6} \mathrm{~N}\right]$.

2-But-3-enyl-2-octyldecylamine: Lithiumaluminiumhydride $\left(\begin{array}{lll}1.84 & \text { g, } & 48.5\end{array}\right.$ $\mathrm{mmol})$ in anhydrous diethylether $(130 \mathrm{~mL}), 2$-but-3-enyl-2-octyldecanenitrile (10.0 g, $31.3 \mathrm{mmol}$ ) in anhydrous diethylether $(30 \mathrm{~mL}$ ) and subsequently $30 \%$ aqueous $\mathrm{NaOH}(20 \mathrm{~mL})$ were allowed to react as was described for 2,2-dibutylhex-5enylamine. Yield $8.56 \mathrm{~g}(85 \%)$ colorless, very viscous oil, b.p. $115^{\circ} \mathrm{C}-120^{\circ} \mathrm{C} / 1 \times$ $10^{-3}$ mbar, $n_{D}^{20}=1.463$. - IR $(\mathrm{KBr}): \tilde{v}=3076.8 \mathrm{w}, 2955.8 \mathrm{~s}, 2926.4 \mathrm{vs}, 2855.4 \mathrm{~s}$, $1736.5 \mathrm{w}, 1640.6 \mathrm{w}, 1467.1 \mathrm{~m}, 1378.2 \mathrm{w}, 1299.3 \mathrm{w}, 1069.9 \mathrm{w}, 992.8 \mathrm{w}, 907.8 \mathrm{~m}$, 814.9 w, 722.0 w, $\mathrm{cm}^{-1} 636.9$ w. ${ }^{1} \mathrm{H}$ NMR $\left(300 \mathrm{MHz}, \mathrm{CDCl}_{3}, 25^{\circ} \mathrm{C}\right): \delta=0.85\left(\mathrm{t},{ }^{3} \mathrm{~J}\right.$ $\left.=6.7 \mathrm{~Hz}, 6 \mathrm{H}, 2 \mathrm{CH}_{3}\right), 1.12-1.24\left(\mathrm{~m}, 30 \mathrm{H}, 15 \mathrm{CH}_{2}\right), 1.85-1.93\left(\mathrm{~m}, 2 \mathrm{H}, \mathrm{CH}_{2}\right)$, $2.42\left(\mathrm{~s}, 2 \mathrm{H}, \mathrm{CH}_{2}-\mathrm{N}\right), 4.85-5.00\left(\mathrm{~m}, 2 \mathrm{H}, \mathrm{CH}_{2, \text { olefin. }}\right), 5.71-5.84 \mathrm{ppm}(\mathrm{m}, 1 \mathrm{H}$, $\left.\mathrm{CH}_{\text {olefin. }}\right) .{ }^{13} \mathrm{C} \mathrm{NMR}\left(75 \mathrm{MHz}, \mathrm{CDCl}_{3}, 25^{\circ} \mathrm{C}\right): \delta=14.0,22.6,22.8,27.5,29.3,29.6$, 30.5, 31.8, 33.7, 34.4, 38.4, $47.1\left(\mathrm{CH}_{2}-\mathrm{N}\right), 113.8\left(\mathrm{CH}_{2 \text {, olefin. }}\right), 139.4 \mathrm{ppm}\left(\mathrm{CH}_{\text {olefin }}\right)$. MS $\left(\mathrm{DEI}^{+} / 70 \mathrm{eV}\right) \mathrm{m} / z(\%): 322(11)\left[M^{+}-\mathrm{H}\right], 308(34)\left[M^{+}-\mathrm{NH}_{2}\right], 294(45)$ $\left[M^{+}-\mathrm{CH}_{4} \mathrm{~N}\right], 210(100)\left[M^{+}-\mathrm{C}_{8} \mathrm{H}_{17}\right], 139(34)\left[M^{+}-\mathrm{C}_{6} \mathrm{H}_{13}-\mathrm{C}_{7} \mathrm{H}_{15}\right], 111$ (52) $\left[M^{+}-\mathrm{C}_{8} \mathrm{H}_{17}-\mathrm{C}_{7} \mathrm{H}_{15}\right], 97(83)\left[M^{+}-2 \times \mathrm{C}_{8} \mathrm{H}_{17}\right], 83(68)\left[M^{+-} 2 \times \mathrm{C}_{8} \mathrm{H}_{17}-\mathrm{NH}_{2}\right]$, 
69 (52) $\left[M^{+}-2 \times \mathrm{C}_{8} \mathrm{H}_{17}-\mathrm{CH}_{4} \mathrm{~N}\right], 55(41)\left[M^{+}-2 \times \mathrm{C}_{8} \mathrm{H}_{17}-\mathrm{C}_{2} \mathrm{H}_{6} \mathrm{~N}\right]$.

2-Butyl-2-vinylhexylamine: Lithiumaluminiumhydride (460 mg, $12.1 \mathrm{mmol}$ ) in anhydrous diethylether (30 mL), 2-butyl-2-vinylhexanenitrile (1.40 g, 7.81 $\mathrm{mmol})$ in anhydrous diethylether $(8 \mathrm{~mL})$ and subsequently $30 \%$ aqueous $\mathrm{NaOH}$ $(5 \mathrm{~mL})$ were allowed to react as was described for 2,2-dibutylhex-5-enylamine. Yield $791 \mathrm{mg}(55 \%)$ colorless liquid, b.p. $43-44^{\circ} \mathrm{C} / 1 \cdot 10^{-3} \mathrm{mbar}, n_{D}^{20}=1.455$. IR $(\mathrm{KBr}): \tilde{v}=3080.8 \mathrm{w}, 2957.2 \mathrm{vs}, 2929.9 \mathrm{vs}, 2860.5 \mathrm{~s}, 1666.3 \mathrm{~m}, 1636.9 \mathrm{w}, 1535.0$ w, $1466.5 \mathrm{~m}, 1414.1 \mathrm{w}, 1378.3 \mathrm{~m}, 1260.9 \mathrm{w}, 1235.9 \mathrm{w}, 1095.9 \mathrm{w}, 1003.9 \mathrm{w}, 910.5$ m, 802.1 w, $729.3 \mathrm{~cm}^{-1}$ w. ${ }^{1} \mathrm{H}$ NMR (300 MHz, $\left.\mathrm{CDCl}_{3}, 25^{\circ} \mathrm{C}\right): \delta=0.86\left(\mathrm{t},{ }^{3} \mathrm{~J}=7.1\right.$ $\left.\mathrm{Hz}, 6 \mathrm{H}, 2 \mathrm{CH}_{3}\right), 1.08-1.32\left(\mathrm{~m}, 12 \mathrm{H}, 6 \mathrm{CH}_{2}\right), 2.49\left(\mathrm{~s}, 2 \mathrm{H}, \mathrm{CH}_{2}-\mathrm{N}\right), 4.86-4.93$ (m, $\left.1 \mathrm{H}, \mathrm{CH}_{2 \text {, olefin. }}\right), 5.07-5.11\left(\mathrm{~m}, 1 \mathrm{H}, \mathrm{CH}_{2 \text {, olefin. }}\right), 5.51-5.60\left(\mathrm{~m}, 1 \mathrm{H}, \mathrm{CH}_{\text {olefin. }}\right)$. ${ }^{13} \mathrm{C} \mathrm{NMR}\left(75 \mathrm{MHz}, \mathrm{CDCl}_{3}, 25^{\circ} \mathrm{C}\right): \delta=14.0,23.5,25.4,33.3,43.9,47.8\left(\mathrm{CH}_{2}-\mathrm{N}\right)$, $113.6\left(\mathrm{CH}_{2, \text { olefin. }}\right), 145.4 \mathrm{ppm}\left(\mathrm{CH}_{\text {olefin. }}\right)$. MS $\left(\mathrm{DEI}^{+} / 70 \mathrm{eV}\right) \mathrm{m} / z(\%): 184(6)\left[M^{+}+\right.$ $\mathrm{H}], 154(43)\left[M^{+}-\mathrm{CH}_{4} \mathrm{~N}\right], 126(100)\left[M^{+}-\mathrm{C}_{4} \mathrm{H}_{9}\right], 112(56)\left[M^{+}-\mathrm{C}_{4} \mathrm{H}_{9}-\mathrm{NH}_{2}\right]$, $70(94)\left[M^{+}-2 \times \mathrm{C}_{4} \mathrm{H}_{9}\right], 55(68)\left[M^{+}-2 \times \mathrm{C}_{4} \mathrm{H}_{9}-\mathrm{NH}_{2}\right], 41(51)\left[M^{+}-2 \times \mathrm{C}_{4} \mathrm{H}_{9}-\right.$ $\left.\mathrm{CH}_{4} \mathrm{~N}\right]$.

2-Allyl-2-butylhexylamine: Lithiumaluminiumhydride $(2.52 \mathrm{~g}, 66.5 \mathrm{mmol})$ in anhydrous diethylether (150 mL), 2-allyl-2-butylhexanenitrile (8.30 g, 42.9 $\mathrm{mmol})$ in anhydrous diethylether $(40 \mathrm{~mL})$ and subsequently $30 \%$ aqueous $\mathrm{NaOH}$ $(20 \mathrm{~mL})$ were allowed to react as was described for 2,2-dibutylhex-5-enylamine. Yield $5.98 \mathrm{~g}(71 \%)$ colorless liquid, b.p. $66^{\circ} \mathrm{C}-68^{\circ} \mathrm{C} / 1 \times 10^{-3} \mathrm{mbar}, n_{D}^{20}=1.459-$ IR $(\mathrm{KBr}): \tilde{v}=3074.4 \mathrm{w}, 2956.9$ vs, $2929.8 \mathrm{vs}, 2861.2 \mathrm{~s}, 1728.5 \mathrm{w}, 1638.2 \mathrm{w}$, $1580.3 \mathrm{w}, 1467.6 \mathrm{~m}, 1379.2 \mathrm{w}, 1299.8 \mathrm{w}, 1103.7 \mathrm{w}, 996.2 \mathrm{w}, 911.4 \mathrm{w}, 815.7 \mathrm{~cm}^{-1}$ w. ${ }^{1} \mathrm{H} \mathrm{NMR}\left(300 \mathrm{MHz}, \mathrm{CDCl}_{3}, 25^{\circ} \mathrm{C}\right): \delta=0.89\left(\mathrm{t},{ }^{3} J=7.0 \mathrm{~Hz}, 6 \mathrm{H}, 2 \mathrm{CH}_{3}\right), 1.15-$ $1.33\left(\mathrm{~m}, 12 \mathrm{H}, 6 \mathrm{CH}_{2}\right), 1.94-1.98\left(\mathrm{~m}, 2 \mathrm{H}, \mathrm{CH}_{2}\right), 2.44\left(\mathrm{~s}, 2 \mathrm{H}, \mathrm{CH}_{2}-\mathrm{N}\right), 4.99-$ $5.06\left(\mathrm{~m}, 2 \mathrm{H}, \mathrm{CH}_{2, \text { olefin. }}\right), 5.70-5.84 \mathrm{ppm}\left(\mathrm{m}, 1 \mathrm{H}, \mathrm{CH}_{\text {olefin. }}\right) .{ }^{13} \mathrm{C} \mathrm{NMR}(75 \mathrm{MHz}$, $\left.\mathrm{CDCl}_{3}, 25^{\circ} \mathrm{C}\right): \delta=14.1,23.6,25.2,34.1,39.4,39.5,47.4\left(\mathrm{CH}_{2}-\mathrm{N}\right), 116.7$ $\left(\mathrm{CH}_{2, \text { olefin. }}\right), 135.1 \mathrm{ppm}\left(\mathrm{CH}_{\text {olefin. }}\right)$. MS $\left(\mathrm{DEI}^{+} / 70 \mathrm{eV}\right) \mathrm{m} / z(\%): 197$ (19) $\left[M^{+}\right], 182$ (100) $\left[M^{+}-\mathrm{NH}_{2}\right], 168(5)\left[M^{+}-\mathrm{CH}_{4} \mathrm{~N}\right], 140(14)\left[M^{+}-\mathrm{C}_{4} \mathrm{H}_{9}\right], 69(3)\left[M^{+}-2 \times\right.$ $\left.\mathrm{C}_{4} \mathrm{H}_{9}-\mathrm{NH}_{2}\right], 57(16)\left[M^{+}-2 \times \mathrm{C}_{4} \mathrm{H}_{9}-\mathrm{CH}_{4} \mathrm{~N}\right]$.

2,2-Dibutylhept-6-enylamine: Lithiumaluminiumhydride (2.35 g, 61.7 $\mathrm{mmol})$ in anhydrous diethylether $(150 \mathrm{~mL}), 2,2$-dibutylhept-6-enenitrile $(8.80 \mathrm{~g}$, $39.8 \mathrm{mmol}$ ) in anhydrous diethylether $(40 \mathrm{~mL})$ and subsequently $30 \%$ aqueous $\mathrm{NaOH}(20 \mathrm{~mL})$ were allowed to react as was described for 2,2-dibutylhex-5enylamine. Yield $7.31 \mathrm{~g}(81 \%)$ colorless liquid, b.p. $82^{\circ} \mathrm{C}-84^{\circ} \mathrm{C} / 2 \times 10^{-3} \mathrm{mbar}$, $n_{D}^{20}=1.461 . \mathrm{IR}(\mathrm{KBr}): \tilde{v}=3076.9 \mathrm{w}, 2956.0 \mathrm{vs}, 2930.1 \mathrm{vs}, 2861.2 \mathrm{~s}, 1871.8 \mathrm{w}$, $1640.3 \mathrm{~m}, 1467.5 \mathrm{~m}, 1378.5 \mathrm{w}, 1297.2 \mathrm{w}, 1069.1 \mathrm{w}, 992.3 \mathrm{w}, 909 . \mathrm{m}, 814.9 \mathrm{w}$, $730.0 \mathrm{w}, 642.7 \mathrm{w}, 556.3 \mathrm{~cm}^{-1} \mathrm{w} .{ }^{1} \mathrm{H}$ NMR $\left(300 \mathrm{MHz}, \mathrm{CDCl}_{3}, 25^{\circ} \mathrm{C}\right): \delta=0.88\left(\mathrm{t},{ }^{3} \mathrm{~J}\right.$ $\left.=7.2 \mathrm{~Hz}, 6 \mathrm{H}, 2 \mathrm{CH}_{3}\right), 1.11-1.29\left(\mathrm{~m}, 16 \mathrm{H}, 8 \mathrm{CH}_{2}\right), 1.97-2.04\left(\mathrm{~m}, 2 \mathrm{H}, \mathrm{CH}_{2}\right)$, 2.43 (s, $\left.2 \mathrm{H}, \mathrm{CH}_{2}-\mathrm{N}\right), 4.91-5.03\left(\mathrm{~m}, 2 \mathrm{H}, \mathrm{CH}_{2 \text {, olefin. }}\right), 5.72-5.86$ ppm (m, $1 \mathrm{H}$, $\left.\mathrm{CH}_{\text {olefin. }}\right) .{ }^{13} \mathrm{C} \mathrm{NMR}\left(75 \mathrm{MHz}, \mathrm{CDCl}_{3}, 25^{\circ} \mathrm{C}\right): \delta=14.1,22.4,23.6,25.2,33.9,34.2$, 34.6, 38.3, $47.2\left(\mathrm{CH}_{2}-\mathrm{N}\right), 114.4\left(\mathrm{CH}_{2, \text { olefin. }}\right), 139.0 \mathrm{ppm}\left(\mathrm{CH}_{\text {olefin. }}\right) . \mathrm{MS}\left(\mathrm{DEI}^{+} / 70\right.$ eV) $m / z(\%): 225(4)\left[M^{+}\right], 194(5)\left[M^{+}-\mathrm{CH}_{4} \mathrm{~N}\right], 168(24)\left[M^{+}-\mathrm{C}_{4} \mathrm{H}_{9}\right], 111(40)$ 
$\left[M^{+}-2 \times \mathrm{C}_{4} \mathrm{H}_{9}\right], 97\left[M^{+}-2 \times \mathrm{C}_{4} \mathrm{H}_{9}-\mathrm{NH}_{2}\right], 83(100)\left[M^{+}-2 \times \mathrm{C}_{4} \mathrm{H}_{9}-\mathrm{CH}_{4} \mathrm{~N}\right]$, 69 (96) $\left[M^{+}-2 \times \mathrm{C}_{4} \mathrm{H}_{9} \mathrm{~N}-\mathrm{C}_{2} \mathrm{H}_{6} \mathrm{~N}\right], 55(100)\left[M^{+}-2 \times \mathrm{C}_{4} \mathrm{H}_{9}-\mathrm{C}_{3} \mathrm{H}_{8} \mathrm{~N}\right], 40$ (29) $\left[M^{+}-2 \times \mathrm{C}_{4} \mathrm{H}_{9}-\mathrm{C}_{4} \mathrm{H}_{10} \mathrm{~N}\right]$.

2,2-Diisopropylbut-3-enylamine: Lithiumaluminiumhydride (366 mg, 9.64 $\mathrm{mmol}$ ) in anhydrous diethylether $(30 \mathrm{~mL}), 2,2$-diisopropylbut-3-enenitrile (940 mg, $6.22 \mathrm{mmol})$ in anhydrous diethylether $(10 \mathrm{~mL})$ and subsequently $30 \%$ aqueous $\mathrm{NaOH}(10 \mathrm{~mL})$ were allowed to react as was described for 2,2-dibutylhex-5enylamine. Yield $282 \mathrm{mg}(29 \%)$ colorless liquid, b.p. $26^{\circ} \mathrm{C} / 3.2 \times 10^{-2} \mathrm{mbar}$. IR $(\mathrm{KBr}): \tilde{v}=3081.6 \mathrm{w}, 2966.2 \mathrm{vs}, 2877.7 \mathrm{~m}, 1660.8 \mathrm{w}, 1634.4 \mathrm{w}, 1466.3 \mathrm{~m}, 1413.8$ w, $1389.7 \mathrm{~m}, 1260.2 \mathrm{w}, 1171.4 \mathrm{w}, 1064.0 \mathrm{w}, 1010.0 \mathrm{w}, 926.1 \mathrm{~m}, 804.8 \mathrm{w}, 698.3 \mathrm{w}$, $671.5 \mathrm{~cm}^{-1} \mathrm{w}$.

2,2-Diisopropylhex-5-enylamine; alternative synthesis: A 70\% solution of sodium-bis(2-methoxyethoxo)aluminiumdihydride in toluene (13.7 g, $47.8 \mathrm{mmol}$ ) under nitrogen atmosphere was diluted with anhydrous toluene $(4 \mathrm{~mL})$, reaed under reflux, treated dropwise within $15 \mathrm{~min}$ with a solution of 2,2-diisopropylhex-5enenitrile $(3.42 \mathrm{~g}, 19.1 \mathrm{mmol})$ in anhydrous toluene $(3 \mathrm{~mL})$, refluxed for $4 \mathrm{~h}$, cooled to $0^{\circ} \mathrm{C}$, treated with $30 \%$ aqueous $\mathrm{NaOH}(15 \mathrm{~mL})$, diluted with distilled water $(100 \mathrm{~mL})$, extracted with diethylether $(3 \times 150 \mathrm{~mL})$, washed with distilled water $(2 \times 200 \mathrm{~mL})$, dried with magnesiumsulphate, evaporated and distilled in vacuo. Yield $2.43 \mathrm{~g}(53 \%)$ colorless liquid, b.p. $98^{\circ} \mathrm{C}-100^{\circ} \mathrm{C} / 22 \mathrm{mbar}, n_{D}^{20}=$ 1.478. IR (KBr): $\tilde{v}=3076.1 \mathrm{w}, 3020.0 \mathrm{w}, 2961.9 \mathrm{vs}, 2880.5 \mathrm{~s}, 1640.4 \mathrm{~m}, 1580.3$ m, 1468.9 m, 1385.0 m, $1296.1 \mathrm{~m}, 1166.9 \mathrm{w}, 1066.7 \mathrm{w}, 992.5 \mathrm{w}, 969.2 \mathrm{w}, 908.6 \mathrm{w}$, $815.7 \mathrm{w}, 721.2 \mathrm{w}, 660.2 \mathrm{~cm}^{-1}$ w. MS $\left(\mathrm{DEI}^{+} / 70 \mathrm{eV}\right) \mathrm{m} / z(\%): 184(3)\left[M^{+}+\mathrm{H}\right], 168$ (4) $\left[M^{+}-\mathrm{NH}_{2}\right], 152(7)\left[M^{+}-\mathrm{CH}_{4} \mathrm{~N}\right], 140(100)\left[M^{+}-\mathrm{C}_{3} \mathrm{H}_{7}\right], 123(23)\left[M^{+}-\right.$ $\left.\mathrm{C}_{3} \mathrm{H}_{7}-\mathrm{NH}_{2}\right], 109(47)\left[M^{+}-\mathrm{C}_{3} \mathrm{H}_{7}-\mathrm{CH}_{4} \mathrm{~N}\right], 97(77)\left[M^{+}-2 \times \mathrm{C}_{3} \mathrm{H}_{7}\right], 83$ (57) $\left[M^{+}-2 \times \mathrm{C}_{3} \mathrm{H}_{7}-\mathrm{NH}_{2}\right], 69(90)\left[M^{+}-2 \times \mathrm{C}_{3} \mathrm{H}_{7}-\mathrm{CH}_{4} \mathrm{~N}\right], 55(95)\left[\mathrm{C}_{4} \mathrm{H}_{7}\right], 43$ (45) $\left[\mathrm{C}_{3} \mathrm{H}_{7}\right]$.

2,9-Bis-(2,2-dibutylhex-5-enyl)anthra[2,1,9-def,6,5,10-d'e'f']diisoquinolin e-1,3,8,10-tetraone (19): Perylene-3,4:9,10-tetracarboxylicbisanhydride (60 mg, $1.18 \mathrm{mmol}$ ) under argon was treated with imidazole $(4.5 \mathrm{~g})$, the quantity of a micro spatulum of zincacetate dihydrate, heated at $140^{\circ} \mathrm{C}$, treated dropwise with 2,2-dibutylhex-5-enylamine $(1.00 \mathrm{~g}, 4.74 \mathrm{mmol})$, stirred at $140^{\circ} \mathrm{C}$ for $4 \mathrm{~h}$, still warm treated with ethanol $(50 \mathrm{~mL})$, precipitated with $2 \mathrm{~N}$ aqueous $\mathrm{HCl}$, diluted with distilled water $(100 \mathrm{~mL})$, extracted with chloroform, dried with magnesiumsulphate, evaporated and purified by column separation ( silica gel, chloroform/ethanol 80:1). Yield $869 \mathrm{mg}(94 \%)$ reddish orange solid with a metallic lustre, m.p. > $250^{\circ} \mathrm{C}$. $R_{\mathrm{f}}$ value (silica gel; $\mathrm{CHCl}_{3} / \mathrm{EtOH} \mathrm{80:1)}=0.45$. $-\mathrm{IR}:(\mathrm{KBr}): \tilde{v}=2956.1 \mathrm{~s}$, 2924.5 vs, 2854.2 vs, $1702.4 \mathrm{~m}, 1661.4 \mathrm{~m}, 1595.7 \mathrm{~m}, 1579.3 \mathrm{w}, 1462.5 \mathrm{~m}, 1405.1$ w, $1378.1 \mathrm{w}, 1335.5 \mathrm{~m}, 1250.6 \mathrm{w}, 1158.2 \mathrm{w}, 1124.8 \mathrm{w}, 907.4 \mathrm{w}, 810.6 \mathrm{w}, 748.5$ $\mathrm{cm}^{-1}$ w. ${ }^{1} \mathrm{H}$ NMR $\left(600 \mathrm{MHz}, \mathrm{CDCl}_{3}, 25^{\circ} \mathrm{C}\right): \delta=0.90\left(\mathrm{t},{ }^{3} \mathrm{~J}=7.1 \mathrm{~Hz}, 12 \mathrm{H}, 4 \mathrm{CH}_{3}\right)$, 1.25 - $1.36\left(\mathrm{~m}, 24 \mathrm{H}, 12 \mathrm{CH}_{2}\right), 1.38$ - 1.41 (m, $\left.4 \mathrm{H}, 2 \mathrm{CH}_{2}\right), 2.11-2.14(\mathrm{~m}, 4 \mathrm{H}, 2$ $\left.\mathrm{CH}_{2}\right), 4.25\left(\mathrm{~s}, 4 \mathrm{H}, 2 \mathrm{~N}-\mathrm{CH}_{2}\right), 4.89-4.19\left(\mathrm{~m}, 2 \mathrm{H}, \mathrm{CH}_{2, \text { olefin. }}\right), 4.99-5.02(2 \mathrm{H}$, $\left.\mathrm{CH}_{2 \text {, olefin. }}\right), 5.79-5.86\left(\mathrm{~m}, 2 \mathrm{H}, \mathrm{CH}_{\text {olefin }}\right), 8.59-8.67 \mathrm{ppm}\left(\mathrm{m}, 8 \mathrm{H}, \mathrm{CH}_{\text {aromat. }}\right) .{ }^{13} \mathrm{C}$ 
NMR (150 MHz, $\left.\mathrm{CDCl}_{3}, 25^{\circ} \mathrm{C}\right): \delta=14.2,23.7,25.7,28.3,35.7,35.9,40.8,45.6$, $113.8,123.0,123.5,126.5,129.3,131.4,134.6,139.8,164.3 \mathrm{ppm}$. UV/Vis $\left(\mathrm{CHCl}_{3}\right)$ $\lambda_{\max }(\varepsilon)=457.5(18700), 488.4(51000), 524.9 \mathrm{~nm}(84700)$. Fluorescence $\left(\mathrm{CHCl}_{3}\right)$ : $\lambda_{\max }\left(I_{\mathrm{rel}}\right)=533(1.00), 575 \mathrm{~nm}(0.36)$. Fluorescence quantum yield $\left(\lambda_{\mathrm{exc}}=488 \mathrm{~nm}\right.$, $E_{483 \mathrm{~nm} / 1 \mathrm{~cm}}=0.0259$, reference: 2,9-bis-(1-hexylheptyl)-anthra[2,1,9-def;6,5,10d'e'f] diisoquinoline-1,3,8,10-tetraone (1) with $\Phi=1.00$ [40]): 0.99. MS: $\left(\mathrm{DEI}^{+} / 70 \mathrm{eV}\right): m / z(\%): 778(100)\left[M^{+}\right], 723(21)\left[M^{+}-\mathrm{C}_{4} \mathrm{H}_{9}\right], 598(27)\left[M^{+}-\right.$ $\left.\mathrm{C}_{13} \mathrm{H}_{25}\right], 585(12)\left[M^{+}-\mathrm{C}_{14} \mathrm{H}_{27}\right], 418$ (67) $\left[M^{+}-2 \times \mathrm{C}_{13} \mathrm{H}_{25}\right], 404$ (25) $\left[M^{+}-\right.$ $\left.\mathrm{C}_{13} \mathrm{H}_{25}-\mathrm{C}_{14} \mathrm{H}_{27}\right], 390(6)\left[M^{+}-2 \times \mathrm{C}_{14} \mathrm{H}_{27}\right] \cdot \mathrm{C}_{52} \mathrm{H}_{62} \mathrm{~N}_{2} \mathrm{O}_{4}$ (778.5): Calcd. C 80.17, $\mathrm{H}$ 8.02, N 3.60; found C 79.99, H 8.05, N 3.62.

2,9-Bis-(2-but-3-enyl-2-hexyloctyl)anthra[2,1,9-def,6,5,10-d'e'f] diisoquin oline-1,3,8,10-tetraone: Perylene-3,4: 9,10-tetracarboxylicbisanhydride (1.00 g, $2.55 \mathrm{mmol}$ ), imidazole (6.0 g), 2-but-3-enyl-2-hexyloctylamine (2.73 g, 10.2 $\mathrm{mmol})$ and ethanol $(15 \mathrm{~mL})$ were allowed to react analogously to 2,9-bis-(2,2dibutylhex-5-enyl)anthra-[2,1,9-def,6,5,10-d'e'f] diisoquinoline-1,3,8,10-tetraone, precipitated with $2 \mathrm{~N}$ aqueous $\mathrm{HCl}(80 \mathrm{~mL})$, extracted with chloroform dried with magnesium sulphate and purified by column separation (silica gel, chloroform, colum $700 \times 54 \mathrm{~mm})$. Yield $1.69 \mathrm{~g}(74 \%)$ bright red glossy solid, m.p. $130^{\circ} \mathrm{C}$. $R_{\mathrm{f}}$-value (silica gel, $\left.\mathrm{CHCl}_{3}\right)=0.52$. IR: $(\mathrm{KBr}): \tilde{v}=30774 \mathrm{w}, 2954.7$ vs, 2929.7 vs, 2857.8 s, 1701.8 s, 1661.5 vs, 1595.6 vs, 1579.0 m, 1507.7 w, 1458.7 m, $1438.4 \mathrm{~m}, 1405.4 \mathrm{~m}, 1377.4 \mathrm{~m}, 1335.8 \mathrm{~s}, 1251.0 \mathrm{~m}, 1216.4 \mathrm{w}, 1178.1 \mathrm{w}, 1159.4$ w, $1125.8 \mathrm{w}, 995.1 \mathrm{w}, 908.7 \mathrm{~m}, 853.5 \mathrm{~m}, 811.2 \mathrm{~m}, 796.0 \mathrm{w}, 750.0 \mathrm{w}, 723.4 \mathrm{w}, 673.9$ w, $633.2 \mathrm{w}, 581.8 \mathrm{~cm}^{-1}$ w. ${ }^{1} \mathrm{H} \operatorname{NMR}\left(600 \mathrm{MHz}, \mathrm{CDCl}_{3}, 25^{\circ} \mathrm{C}\right): \delta=0.87\left(\mathrm{t},{ }^{3} J=6.8\right.$ $\left.\mathrm{Hz}, 12 \mathrm{H}, 4 \mathrm{CH}_{3}\right), 1.26-1.33\left(\mathrm{~m}, 40 \mathrm{H}, 20 \mathrm{CH}_{2}\right), 1.37-1.40\left(\mathrm{~m}, 4 \mathrm{H}, 2 \mathrm{CH}_{2}\right), 2.10$ - $2.14\left(\mathrm{~m}, 4 \mathrm{H}, 2 \mathrm{CH}_{2}\right), 4.24$ (s, $\left.4 \mathrm{H}, \mathrm{N}-\mathrm{CH}_{2}\right), 4.89$ - 4.91 (m, $2 \mathrm{H}, \mathrm{CH}_{2 \text {, olefin. }), 4.98}$ - $5.01\left(2 \mathrm{H}, \mathrm{CH}_{2, \text { olefin. }}\right), 5.79$ - $5.85\left(\mathrm{~m}, 2 \mathrm{H}, \mathrm{CH}_{\text {olefin. }}\right), 8.54-8.63 \mathrm{ppm}(\mathrm{m}, 8 \mathrm{H}$, $\left.\mathrm{CH}_{\text {aromat. }}\right) .{ }^{13} \mathrm{C} \mathrm{NMR}\left(150 \mathrm{MHz}, \mathrm{CDCl}_{3}, 25^{\circ} \mathrm{C}\right): \delta=13.9,22.5,23.3,27.9,30.2$, 31.7, 35.3, 36.0, 40.5, 45.3, 113.6, 122.8, 123.3, 126.2, 129.0, 131.3, 134.3, 139.7, 164.0 ppm. UV/Vis $\left(\mathrm{CHCl}_{3}\right) \lambda_{\max }(E)=457.7(0.22), 488.7(0.60), 525.2 \mathrm{~nm}$ (1.00). Fluorescence $\left(\mathrm{CHCl}_{3}\right): \lambda_{\max }\left(I_{\text {rel }}\right)=533(1.00), 575 \mathrm{~nm}(0.36)$. Fluorescence quantum yield $\left(\lambda_{\mathrm{exc}}=488 \mathrm{~nm}, E_{483 \mathrm{~nm} / 1 \mathrm{~cm}}=0.0401\right.$, reference: 2,9 -bis-( 1 - hexylheptyl)anthra[2,1,9-def;6,5,10-d'e'f] diisoquinoline- 1,3,8,10-tetraone (1) with $\Phi$ $=1.00$ [40]): 1.00. MS: $\left(\mathrm{DEI}^{+} / 70 \mathrm{eV}\right): \mathrm{m} / z(\%): 890(100)\left[M^{+}\right], 835$ (22) $\left[M^{+}-\right.$ $\left.\mathrm{C}_{4} \mathrm{H}_{7}\right], 654$ (39) $\left[M^{+}-\mathrm{C}_{17} \mathrm{H}_{33}\right], 641$ (17) $\left[M^{+}-\mathrm{C}_{18} \mathrm{H}_{35}\right], 599(8)\left[M^{+}-\mathrm{C}_{18} \mathrm{H}_{35}-\right.$ $\left.\mathrm{C}_{3} \mathrm{H}_{7}\right], 418(62)\left[M-2 \times \mathrm{C}_{17} \mathrm{H}_{33}\right], 404$ (31) $\left[M^{+}-\mathrm{C}_{17} \mathrm{H}_{33}-\mathrm{C}_{18} \mathrm{H}_{35}\right], 391$ (11) $\left[M^{+}\right.$ $-2 \times \mathrm{C}_{18} \mathrm{H}_{35}$ ].

2,9-Bis-(2-but-3-enyl-2-octyldecyl)anthra[2,1,9-def,6,5,10-d'e'f] diisoquin oline-1,3,8,10-tetraone: Perylene-3,4: 9,10-tetracarboxylicbisanhydride (500 $\mathrm{mg}, 1.27 \mathrm{mmol}$ ), imidazole (3.5 g), 2-but-3-enyl-2-octyldecylamine (65 g, 5.10 $\mathrm{mmol}$ ) and ethanol (15 mL) were allowed to react analogously to 2,9-bis-(2-but3-enyl-2-hexyloctyl)anthra-[2,1,9-def,6,5,10-d'e'f] diisoquinoline-1,3,8,10-tetrao ne. The reaction product was purified by column separation (silica gel, chloroform). Yield $875 \mathrm{mg}$ (69\%) dark red, glossy solid, m.p. $105^{\circ} \mathrm{C}$. $R_{\mathrm{f}}$ (silica gel, 
$\mathrm{CHCl}_{3} /$ iso-hexane 10:1) $=0.63 . \mathrm{IR}(\mathrm{KBr}): \tilde{v}=3078.7 \mathrm{w}, 2919.3 \mathrm{vs}, 2852.5 \mathrm{~s}$, $1688.5 \mathrm{~m}, 1651.6 \mathrm{~s}, 1593.3 \mathrm{~m}, 1577.3 \mathrm{~m}, 1506.5 \mathrm{w}, 1482.0 \mathrm{w}, 1453.6 \mathrm{w}, 1435.0 \mathrm{~m}$, $1402.9 \mathrm{~m}, 1378.4 \mathrm{w}, 1351.2 \mathrm{~m}, 1331.8 \mathrm{~s}, 1247.7 \mathrm{~m}, 1215.3 \mathrm{w}, 1174.0 \mathrm{w}, 1155.0 \mathrm{w}$, $1121.4 \mathrm{w}, 1007.1 \mathrm{w}, 900.0 \mathrm{~m}, 873.3 \mathrm{w}, 849.8 \mathrm{~m}, 810.0 \mathrm{~m}, 795.6 \mathrm{w}, 748.4 \mathrm{~m}, 721.7$ $\mathrm{w}, 658.2 \mathrm{~cm}^{-1}$ w. ${ }^{1} \mathrm{H} \mathrm{NMR}\left(600 \mathrm{MHz} \mathrm{CDCl}_{3}, 25^{\circ} \mathrm{C}\right): \delta=0.86\left(\mathrm{t},{ }^{3} J=6.9 \mathrm{~Hz}, 12 \mathrm{H}\right.$, $\left.4 \mathrm{CH}_{3}\right), 1.24$ - $1.32\left(\mathrm{~m}, 56 \mathrm{H}, 28 \mathrm{CH}_{2}\right), 1.38-1.40\left(\mathrm{~m}, 4 \mathrm{H}, 2 \mathrm{CH}_{2}\right), 2.10-2.14$ (m, $\left.4 \mathrm{H}, 2 \mathrm{CH}_{2}\right), 4.23\left(\mathrm{~s}, 4 \mathrm{H}, \mathrm{N}-\mathrm{CH}_{2}\right), 4.83-4.91$ (m, $\left.2 \mathrm{H}, \mathrm{CH}_{2 \text {, olefin }}\right), 4.98-5.02(\mathrm{~m}$, $\left.2 \mathrm{H}, \mathrm{CH}_{2 \text {, olefin. }}\right), 5.79-5.86\left(\mathrm{~m}, 2 \mathrm{H}, \mathrm{CH}_{\text {olefin. }}\right), 8.53-8.62 \mathrm{ppm}\left(\mathrm{m}, 8 \mathrm{H}, \mathrm{CH}_{\mathrm{ar} .}\right) .{ }^{13} \mathrm{C}$ NMR $\left(150 \mathrm{MHz}, \mathrm{CDCl}_{3}, 25^{\circ} \mathrm{C}\right): \delta=14.1,22.7,23.5,28.2,29.4,29.6,30.7,31.9$, $35.6,36.2$, 40.7, 44.6, 113.8, 123.0, 123.5, 126.3, 129.2, 131.4, 134.4, 139.8, 164.2 ppm. UV/Vis $\left(\mathrm{CHCl}_{3}\right) \lambda_{\max }(\varepsilon)=457.0$ (19300), 488.8 (52200), $524.0 \mathrm{~nm}(85100)$. Fluorescence $\left(\mathrm{CHCl}_{3}\right): \lambda_{\max }\left(I_{\text {rel }}\right)=534(1.00), 576.3(0.52), 621.3 \mathrm{~nm}(0.12)$. Fluorescence quantum yield $\left(\lambda_{\text {exc }}=488 \mathrm{~nm}, E_{488 \mathrm{~nm} / 1 \mathrm{~cm}}=0.0256\right.$, reference: 2,9-bis-(1-hexylheptyl)anthra[2,1,9-def,6,5,10-d'e'f] diiso-quinoline-1,3,8,10-tetr aone (1) with $\Phi=1.00$ [40]): 1.00. MS: $\left(\mathrm{DEI}^{+} / 70 \mathrm{eV}\right): \mathrm{m} / z(\%): 1002(100)\left[M^{+}-\right.$ $\mathrm{H}], 947(23)\left[M^{+}-\mathrm{C}_{4} \mathrm{H}_{7}\right], 710(45)\left[M^{+}-\mathrm{C}_{21} \mathrm{H}_{41}\right], 697(20)\left[M^{+}-\mathrm{C}_{22} \mathrm{H}_{43}\right], 418$ (72) $\left[M^{+}-2 \times \mathrm{C}_{21} \mathrm{H}_{41}\right], 404(35)\left[M^{+}-\mathrm{C}_{21} \mathrm{H}_{41}-\mathrm{C}_{22} \mathrm{H}_{43}\right], 391$ (12) $\left[M^{+}-2 \times\right.$ $\left.\mathrm{C}_{22} \mathrm{H}_{43}\right] . \mathrm{C}_{68} \mathrm{H}_{94} \mathrm{~N}_{2} \mathrm{O}_{4}$ (1003.5): Calcd. C 81.39, H 9.44, N 2.79; found $\mathrm{C} \mathrm{81.17,} \mathrm{H}$ 9.57, N 2.99.

2,9-Bis-(2-butyl-2-vinylhexyl)anthra[2,1,9-def,6,5,10-d'e'f] diisoquinoline -1,3,8,10-tetraone: Perylene-3,4: 9,10-tetracarboxylicbisanhydride (658 mg, 1.68 $\mathrm{mmol}$ ), imidazole (6.0 g), 2-butyl-2-vinylhexylamine (700 $\mathrm{mg}, 3.82 \mathrm{mmol}$ ) and ethanol $(25 \mathrm{~mL})$ were allowed to react analogously to 2,9-bis-(2,2-dibutylhex-5enyl)anthra[2,1,9-def,6,5,10- d'e'f] diisoquinoline-1,3,8,10-tetraone. The reaction product was purified by column separation (silica gel, chloroform/ethanol 60:1). Yield $928 \mathrm{mg}$ (78\%) reddish orange, sparingly soluble powder, m.p. $>250^{\circ} \mathrm{C} . R_{\mathrm{f}}$ value (silica gel, $\mathrm{CHCl}_{3} / \mathrm{EtOH} \mathrm{60:1)}=0.73$. IR $(\mathrm{KBr}): \tilde{v}=3080.6 \mathrm{w}, 2956.6 \mathrm{~m}$, $2932.1 \mathrm{~m}, 2870.5 \mathrm{~m}, 1700.9$ vs, 1660.3 vs, 1595.0 s, $1578.8 \mathrm{~m}, 1507.9$ w, $1458.1 \mathrm{w}$, $1438.0 \mathrm{~m}, 1404.5 \mathrm{~m}, 1356.6 \mathrm{~m}, 1335.6 \mathrm{~s}, 1253.0 \mathrm{~m}, 1218.5 \mathrm{w}, 1165.8 \mathrm{w}, 1120.1 \mathrm{w}$, $1010.2 \mathrm{w}, 913.2 \mathrm{w}, 853.1 \mathrm{w}, 810.8 \mathrm{~m}, 796.0 \mathrm{w}, 755.5 \mathrm{~cm}^{-1}$ w. ${ }^{1} \mathrm{H}$ NMR (600 MHz, $\left.\mathrm{CDCl}_{3}, 25^{\circ} \mathrm{C}\right): \delta=0.95\left(\mathrm{t},{ }^{3} J=7.3 \mathrm{~Hz}, 12 \mathrm{H}, 4 \mathrm{CH}_{3}\right), 1.22-1.46(\mathrm{~m}, 24 \mathrm{H}, 12$ $\left.\mathrm{CH}_{2}\right), 4.26\left(\mathrm{~s}, 4 \mathrm{H}, 2 \mathrm{CH}_{2}\right), 4.74-4.76\left(\mathrm{~m}, 2 \mathrm{H}, \mathrm{CH}_{2, \text { olefin. }}\right), 4.88-4.90(\mathrm{~m}, 2 \mathrm{H}$, $\left.\mathrm{CH}_{2 \text {, olefin. }}\right), 5.81-5.86\left(\mathrm{~m}, 2 \mathrm{H}, \mathrm{CH}_{2}\right), 8.59-8.65 \mathrm{ppm}\left(\mathrm{m}, 8 \mathrm{H}, \mathrm{CH}_{\text {aromat. }}\right){ }^{13} \mathrm{C}$ NMR (150 MHz, $\left.\mathrm{CDCl}_{3}, 25^{\circ} \mathrm{C}\right): \delta=14.2,23.6,23.8,25.7,34.0,44.7,45.9,112.6$, $123.1,123.5,126.5,129.2,131.5,134.6,145.0,164.1 \mathrm{ppm}$. UV/Vis $\left(\mathrm{CHCl}_{3}\right) \lambda_{\max }$ $(\varepsilon)=457.1$ (19400), 487.8 (52700), $524.4 \mathrm{~nm}$ (87900). Fluorescence $\left(\mathrm{CHCl}_{3}\right): \lambda_{\max }$ $\left(I_{\text {rel }}\right)=532.8(1.00), 574.3(0.52), 624.3 \mathrm{~nm}(0.14)$. Fluorescence quantum yield $(\lambda \operatorname{exc}=487 \mathrm{~nm}$, E $487 \mathrm{~nm} / 1 \mathrm{~cm}=0.0319$, reference: 2,9-bis-(1-h-exylheptyl)anthra[2,1,9-def ;6,5,10-d'e'f' ]di-isoquinoline-1,3,8,10-tetraone (1) with $\Phi=$ 1.00 [40]): 1.00. MS: (DEI $\left.{ }^{+} / 70 \mathrm{eV}\right): \mathrm{m} / z(\%): 722(65)\left[M^{+}\right], 666(43)\left[M^{+}-\right.$ $\left.\mathrm{C}_{4} \mathrm{H}_{9}\right], 569(100)\left[M^{+}-\mathrm{C}_{11} \mathrm{H}_{21}\right], 557(69)\left[M^{+}-\mathrm{C}_{12} \mathrm{H}_{23}\right], 500(23)\left[M^{+}-\mathrm{C}_{12} \mathrm{H}_{23}-\right.$ $\left.\mathrm{C}_{4} \mathrm{H}_{9}\right], 416(25)\left[M^{+}-2 \times \mathrm{C}_{11} \mathrm{H}_{21}\right], 404(87)\left[M^{+}-\mathrm{C}_{11} \mathrm{H}_{21}-\mathrm{C}_{12} \mathrm{H}_{23}\right], 390(62)\left[M^{+}\right.$ $-2 \times \mathrm{C}_{12} \mathrm{H}_{23}$ ]. $-\mathrm{C}_{48} \mathrm{H}_{54} \mathrm{~N}_{2} \mathrm{O}_{4}$ (723.0): Calcd. C 79.74, H 7.53, N 3.87; found $\mathrm{C}$ 
79.47, H 7.59, N 3.78.

2,9-Bis-(2-allyl-2-butylhexyl)anthra[2,1,9-def,6,5,10-d'e'f] diisoquinoline1,3,8,10-tetraone (17): Perylene-3,4: 9,10-tetracarboxylicbisanhydride (1.00 g, $2.55 \mathrm{mmol})$, imidazole $(6.0 \mathrm{~g}), 2$-allyl-2-butyl-hexylamine $(2.01 \mathrm{~g}, 10.2 \mathrm{mmol})$ and ethanol $(15 \mathrm{~mL})$ were allowed to react analogously to 2,9-bis-(2,2dibutylhex-5-enyl)anthra[2,1,9-def,6,5,10- d'e'f] diisoquinoline-1,3,8,10-tetraone. The reaction product was purified by column separation (silica gel, chloroform/ethanol 60:1). Yield $1.43 \mathrm{~g}(75 \%)$ bright orange powder, m.p. $>250^{\circ} \mathrm{C} . R_{\mathrm{f}}$ value (silica gel; $\mathrm{CHCl}_{3} / \mathrm{EtOH} \mathrm{60:1)}=0.53$. IR: $(\mathrm{KBr}): \tilde{v}=3071.5 \mathrm{w}, 2952.4 \mathrm{~m}$, 2929.4 m, 2867.9 m, 1691.4 s, 1650.2 vs, 1591.5 s, 1576.3 m, 1506.0 w, 1464.4 w, 1435.9 m, 1401.9 m, $1386.2 \mathrm{w}, 1370.1 \mathrm{w}, 1357.6 \mathrm{w}, 1333.8 \mathrm{~s}, 1248.0 \mathrm{~m}, 1216.0 \mathrm{w}$, $1200.0 \mathrm{w}, 1158.2 \mathrm{w}, 1125.8 \mathrm{w}, 1103.1 \mathrm{w}, 1071.7 \mathrm{w}, 1016.7 \mathrm{w}, 1001.9 \mathrm{w}, 988.5 \mathrm{w}$, 970.5 w, 898.8 w, 868.6 w, 851.8 w, 810.3 m, 793.3 w, 747.6 m, 710.5 w, 658.9 $\mathrm{cm}^{-1}$ w. ${ }^{1} \mathrm{H} \mathrm{NMR}\left(600 \mathrm{MHz}, \mathrm{CDCl}_{3}, 25^{\circ} \mathrm{C}\right): \delta=0.90\left(\mathrm{t},{ }^{3} J=7.1 \mathrm{~Hz}, 12 \mathrm{H}, 4 \mathrm{CH}_{3}\right)$, $1.26-1.38\left(\mathrm{~m}, 24 \mathrm{H}, 12 \mathrm{CH}_{2}\right), 2.14\left(\mathrm{~d},{ }^{3} J=7.1 \mathrm{~Hz}, 4 \mathrm{H}, 2 \mathrm{CH}_{2}\right), 4.26(\mathrm{~s}, 4 \mathrm{H}, 2$ $\left.\mathrm{N}-\mathrm{CH}_{2}\right), 4.92-4.94\left(\mathrm{~m}, 2 \mathrm{H}, \mathrm{CH}_{2}\right.$, olefin. $), 5.00-5.02\left(\mathrm{~m}, 2 \mathrm{H}, \mathrm{CH}_{2, \text { olefin. }}\right), 5.90$ $5.97\left(\mathrm{~m}, 2 \mathrm{H}, \mathrm{CH}_{\text {olefin. }}\right), 8.60-8.66 \mathrm{ppm}\left(\mathrm{m}, 8 \mathrm{H}, \mathrm{CH}_{\text {aromat }}\right) .{ }^{13} \mathrm{C} \mathrm{NMR}(150 \mathrm{MHz}$, $\left.\mathrm{CDCl}_{3}, 25^{\circ} \mathrm{C}\right): \delta=16.4,25.9,27.8,38.3,43.0,43.1,48.0,119.0,125.2,125.7$, 128.7, 131.5, 133.7, 136.8, 137.4, $166.6 \mathrm{ppm}$. UV/Vis $\left(\mathrm{CHCl}_{3}\right) \lambda_{\max }(E)=458.2$ (0.22), $489.1(0.60), 525.6 \mathrm{~nm}(1.00)$. Fluorescence $\left(\mathrm{CHCl}_{3}\right): \lambda_{\max }\left(I_{\text {rel }}\right)=534.5$ (1.00), $577.0(0.52), 621.8 \mathrm{~nm}(0.12)$. Fluorescence quantum yield $\left(\lambda_{\text {exc }}=488 \mathrm{~nm}\right.$, $E_{488 \mathrm{~nm} / 1 \mathrm{~cm}}=0.0323$, reference: 2,9-bis-(1-hexylheptyl)anthra [2,1,9-def,6,5,10$\left.d^{\prime} e^{\prime} f\right]$ diisoquinoline-1,3,8,10-tetraone (1) with $\Phi=1.00$ [40]): 1.00. MS: $\left(\mathrm{DEI}^{+} / 70 \mathrm{eV}\right): \mathrm{m} / z(\%): 750(99)\left[M^{+}\right], 709(57)\left[M^{+}-\mathrm{C}_{3} \mathrm{H}_{5}\right], 584(32)\left[M^{+}-\right.$ $\left.\mathrm{C}_{12} \mathrm{H}_{23}\right], 571(34)\left[M^{+}-\mathrm{C}_{13} \mathrm{H}_{25}\right], 543$ (12).

2,9-Bis-(2,2-dibutylhept-6-enyl)anthra[2,1,9-def,6,5,10-d'e'f] diisoquinoli ne-1,3,8,10-tetraone (21): Perylene-3,4: 9,10-tetracarboxylicbisanhydride (1.00 g, $2.55 \mathrm{mmol})$, imidazole $(5.0 \mathrm{~g}), 2,2$-dibutylhept-6-enylamine $(2.30 \mathrm{~g}, 10.2$ $\mathrm{mmol})$ and ethanol $(25 \mathrm{~mL})$ were allowed to react analogously to 2,9-bis-(2,2dibutylhex-5-enyl)anthra[2,1,9-def,6,5,10- d'e'f] diisoquinoline-1,3,8,10-tetraone. The reaction product was purified by column separation (silica gel, chloroform/ethanol 100:1). Yield $1.77 \mathrm{~g}(86 \%)$ reddish orange small plates, m.p. $239^{\circ} \mathrm{C}$. $R_{\mathrm{f}}$ value (silica gel, $\left.\mathrm{CHCl}_{3}\right)=0.49$. IR $(\mathrm{KBr}): \tilde{v}=3076.8 \mathrm{w}, 2955.6 \mathrm{~m}, 2932.4 \mathrm{~m}$, $2863.8 \mathrm{~m}, 1698.7 \mathrm{vs}, 1660.2 \mathrm{vs}, 1594.8 \mathrm{~s}, 1578.5 \mathrm{~m}, 1507.9 \mathrm{w}, 1457.1 \mathrm{w}, 1438.7 \mathrm{~m}$, $1404.7 \mathrm{~m}, 1335.9 \mathrm{~s}, 1252.4 \mathrm{~m}, 1217.1 \mathrm{w}, 1160.6 \mathrm{w}, 1125.6 \mathrm{w}, 1102.8 \mathrm{w}, 1013.0 \mathrm{w}$, $909.1 \mathrm{w}, 854.2 \mathrm{w}, 811.4 \mathrm{~m}, 796.0 \mathrm{w}, 752.1 \mathrm{w}, 632.9 \mathrm{~cm}^{-1}$ w. ${ }^{1} \mathrm{H}$ NMR $(600 \mathrm{MHz}$, $\left.\mathrm{CDCl}_{3}, 25^{\circ} \mathrm{C}\right): \delta=0.89\left(\mathrm{t},{ }^{3} \mathrm{~J}=7.0 \mathrm{~Hz}, 12 \mathrm{H}, 4 \mathrm{CH}_{3}\right), 1.25-1.32(\mathrm{~m}, 28 \mathrm{H}, 14$ $\left.\mathrm{CH}_{2}\right), 1.42-1.47\left(\mathrm{~m}, 4 \mathrm{H}, 2 \mathrm{CH}_{2}\right), 2.00-2.03\left(\mathrm{~m}, 4 \mathrm{H}, 2 \mathrm{CH}_{2}\right), 4.23(\mathrm{~s}, 4 \mathrm{H}$, $\left.\mathrm{N}-\mathrm{CH}_{2}\right), 4.89-4.91\left(\mathrm{~m}, 2 \mathrm{H}, \mathrm{CH}_{2 \text {, olefin. }}\right), 4.96-4.99\left(\mathrm{~m}, 2 \mathrm{H}, \mathrm{CH}_{2 \text {, olefin. }}\right), 5.78$ 5.85 (m, $\left.2 \mathrm{H}, \mathrm{CH}_{\text {olefin. }}\right), 8.53-8.62 \mathrm{ppm}\left(\mathrm{m}, 8 \mathrm{H}, \mathrm{CH}_{\text {aromat }}\right) .{ }^{13} \mathrm{C} \mathrm{NMR}(150 \mathrm{MHz}$, $\left.\mathrm{CDCl}_{3}, 25^{\circ} \mathrm{C}\right): \delta=14.2,23.1,23.8,25.7,34.8,35,8,40.7,45.6,114.2,123.0,123.5$, $126.4,129.2,131.3,134.4,139.2,164.2 \mathrm{ppm}$. UV/Vis $\left(\mathrm{CHCl}_{3}\right) \lambda_{\max }(E)=457.1$ (0.22), $488.1(0.60), 524.5 \mathrm{~nm}$ (1.00). Fluorescence $\left(\mathrm{CHCl}_{3}\right): \lambda_{\max }\left(I_{\mathrm{rel}}\right)=533.0$ 
(1.00), $574.5(0.52), 623.5 \mathrm{~nm}(0.12)$. Fluorescence quantum yield $\left(\lambda_{\mathrm{exc}}=487 \mathrm{~nm}\right.$, $E_{487 \mathrm{~nm} / 1 \mathrm{~cm}}=0.0296,2$,9-bis-(1-hexyl- heptyl)anthra[2,1,9-def;6,5,10-d'e'f] diisoquinoline-1,3,8,10-tetraone (1) with $\Phi=1.00$ [40]): 1.00. MS: $\left(\mathrm{DEI}^{+} / 70 \mathrm{eV}\right): \mathrm{m} / z$ (\%): $806(100)\left[M^{+}-\mathrm{H}\right], 612(43)\left[M^{+}-\mathrm{C}_{14} \mathrm{H}_{27}\right], 599(11)\left[M^{+}-\mathrm{C}_{15} \mathrm{H}_{29}\right], 418$ (56) $\left[M^{+}-2 \times \mathrm{C}_{14} \mathrm{H}_{27}\right], 404$ (19) $\left[M^{+}-\mathrm{C}_{14} \mathrm{H}_{27}-\mathrm{C}_{15} \mathrm{H}_{29}\right], 391$ (6) $\left[M^{+}-2 \times\right.$ $\mathrm{C}_{15} \mathrm{H}_{27}$ ]. $\mathrm{C}_{54} \mathrm{H}_{66} \mathrm{~N}_{2} \mathrm{O}_{4}$ (807.1): Calcd. C 80.36, H 8.24, N 3.47; fund C 79.94, $\mathrm{H}$ 8.11, N 3.48 .

2,9-Bis-(2,2-diisopropylbut-3-enyl)anthra[2,1,9-def,6,5,10-d'e'f] diisoquin oline-1,3,8,10-tetraone: Perylene-3,4: 9,10-tetracarboxylicbisanhydride (176 $\mathrm{mg}, 450 \mu \mathrm{mol}$ ), imidazole (1.5 g), 2,2-diisopropylbut-3-enylamine (280 mg, 1.80 $\mathrm{mmol})$ and ethanol $(15 \mathrm{~mL})$ were allowed to react analogously to 2,9-bis-(2,2dibutylhex-5-enyl)anthra-[2,1,9-def,6,5,10-d'e'f] diisoquinoline-1,3,8,10-tetraone. The reaction product was purified by column separation (silica gel, chloroform/ethanol 40:1). Yield $13 \mathrm{mg}$ (4\%) sparingly soluble brick red solid, m.p. > $250^{\circ} \mathrm{C}$. $R_{\mathrm{f}}$ value (silica gel; $\left.\mathrm{CHCl}_{3} / \mathrm{EtOH} 40: 1\right)=0.80 . \mathrm{IR}(\mathrm{KBr}): \tilde{v}=3079.2 \mathrm{w}$ br., $2958.1 \mathrm{~m}, 2877.2 \mathrm{~m}, 1697.5$ s, 1651.3 vs, $1591.2 \mathrm{~s}, 1578.0 \mathrm{~m}, 1506.9$ w, 1465.3 w, $1434.6 \mathrm{~m}, 1404.1 \mathrm{~m}, 1379.0 \mathrm{~m}, 1359.5 \mathrm{~m}, 1331.8 \mathrm{~s}, 1246.8 \mathrm{~m}, 1214.2 \mathrm{w}, 1161.9$ w, 1127.1 w, 1099.0 w, 1004.3 w, 913.1 w, 850.6 w, 809.8 m, 792.9 w, $751.8 \mathrm{~m}$, $711.9 \mathrm{~cm}^{-1}$ w. ${ }^{1} \mathrm{H}$ NMR $\left(600 \mathrm{MHz}, \mathrm{CDCl}_{3}, 25^{\circ} \mathrm{C}\right): \delta=0.95-1.00(\mathrm{~m}, 24 \mathrm{H}, 8$ $\left.\mathrm{CH}_{3}\right), 2.15$ - $2.20\left(\mathrm{~m}, 4 \mathrm{H}, 4 \mathrm{CH}_{\text {aliphat. }}\right), 4.48\left(\mathrm{~s}, 4 \mathrm{H}, 2 \mathrm{~N}-\mathrm{CH}_{2}\right), 4.96-4.99(\mathrm{~m}, 2 \mathrm{H}$, $\mathrm{CH}_{2 \text {, olefin. }}$ ), 5.18 - 5.20 (m, $2 \mathrm{H}, \mathrm{CH}_{2 \text {, olefin. }}$ ), 5.92 - 5.97 (m, $2 \mathrm{H}, \mathrm{CH}_{\text {olefin. }}$ ), 8.64 - 8.72 ppm $\left(\mathrm{m}, 8 \mathrm{H}, \mathrm{CH}_{\text {aromat. }}\right)$. UV/Vis $\left(\mathrm{CHCl}_{3}\right) \lambda_{\max }(E)=457.8(0.22), 488.6(0.60)$, $525.0 \mathrm{~nm}$ (1.00). Fluorescence $\left(\mathrm{CHCl}_{3}\right): \lambda_{\max }\left(I_{\text {rel }}\right)=534.5$ (1.00), 577.0 (0.52), $635.8 \mathrm{~nm}$ (0.12). - Fluorescence quantum yield $\left(\lambda_{\mathrm{exc}}=488 \mathrm{~nm}, E_{483 \mathrm{~nm} / 1 \mathrm{~cm}}=\right.$ 0.0318, reference: 2,9-bis-(1-hexylheptyl)anthra[2,1,9-def; 6,5,10-d'e'f]-diiso-

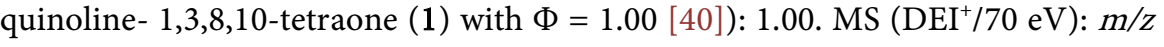
(\%): $666(22)\left[M^{+}\right], 623(23)\left[M^{+}-\mathrm{C}_{3} \mathrm{H}_{7}\right], 541(35)\left[M^{+}-\mathrm{C}_{9} \mathrm{H}_{17}\right], 529$ (35) $\left[M^{+}-\right.$ $\left.\mathrm{C}_{10} \mathrm{H}_{19}\right], 418(19)\left[M^{+}-2 \times \mathrm{C}_{9} \mathrm{H}_{17}\right], 404(98)\left[M^{+}-\mathrm{C}_{9} \mathrm{H}_{17}-\mathrm{C}_{10} \mathrm{H}_{19}\right], 391$ (100) $\left[M^{+}-2 \times \mathrm{C}_{10} \mathrm{H}_{19}\right]$.

\section{2,9-Bis-(2,2-diisopropylhex-5-enyl)anthra[2,1,9-def,6,5,10-d'e'f] diisoqui} noline-1,3,8,10-tetraone: Perylene-3,4: 9,10-tetracarboxylicbisanhydride (100 $\mathrm{mg}, 255 \mu \mathrm{mol}$ ), imidazole (2.0 g), 2,2-diisopropylhex-5-enylamine (140 mg, 765 $\mu \mathrm{mol})$ and ethanol $(15 \mathrm{~mL})$ were allowed to react analogously to 2,9-bis-(2,2dibutylhex-5-enyl)anthra[2,1,9-def,6,5,10-d'e'f] diisoquinoline-1,3,8,10-tetraone. The reaction product was purified by column separation (silica gel, chloroform/ ethanol 60:1). Yield $70 \mathrm{mg}(38 \%)$ brick red solid, m.p. $>250^{\circ} \mathrm{C}$. $R_{\mathrm{f}}$ value (silica gel; $\mathrm{CHCl}_{3} / \mathrm{EtOH}$ 60:1) = 0.74. IR $(\mathrm{KBr}): \tilde{v}=2964.3 \mathrm{~m}, 2880.1 \mathrm{~m}, 1694.6 \mathrm{~s}$, 1650.5 vs, $1591.2 \mathrm{~s}, 1577.8 \mathrm{~m}, 1507.5 \mathrm{w}, 1434.5 \mathrm{w}, 1404.2 \mathrm{~m}, 1379.2 \mathrm{w}, 1358.9 \mathrm{w}$, $1334.4 \mathrm{~s}, 1248.5 \mathrm{~m}, 1214.9 \mathrm{w}, 1162.8 \mathrm{w}, 1128.1 \mathrm{w}, 1100.0 \mathrm{w}, 1039.9 \mathrm{w}, 972.0 \mathrm{w}$, $906.2 \mathrm{w}, 851.4 \mathrm{w}, 810.1 \mathrm{~m}, 793.5 \mathrm{w}, 750.3 \mathrm{~m}, 712.1 \mathrm{~cm}^{-1} \mathrm{w} .{ }^{1} \mathrm{H}$ NMR $(600 \mathrm{MHz}$, $\left.\mathrm{CDCl}_{3}, 25^{\circ} \mathrm{C}\right): \delta=0.98-1.05\left(\mathrm{~m}, 24 \mathrm{H}, 8 \mathrm{CH}_{3}\right), 1.20-1.27\left(\mathrm{~m}, 8 \mathrm{H}, 4 \mathrm{CH}_{2}\right), 2.16$ - $2.22\left(\mathrm{~m}, 4 \mathrm{H}, 4 \mathrm{CH}_{\text {aliphat. }}\right), 4.52\left(\mathrm{~s}, 4 \mathrm{H}, 2 \mathrm{~N}-\mathrm{CH}_{2}\right), 4.84-4.86\left(\mathrm{~m}, 2 \mathrm{H}, \mathrm{CH}_{2, \text { olefin. }}\right)$, 4.91 - $4.94\left(\mathrm{~m}, 2 \mathrm{H}, \mathrm{CH}_{2}\right), 5.36-5.40\left(\mathrm{~m}, 2 \mathrm{H}, \mathrm{CH}_{\text {olefin. }}\right), 8.63-8.71 \mathrm{ppm}(\mathrm{m}, 8 \mathrm{H}$, 
$\left.\mathrm{CH}_{\text {aromat. }}\right)$. UV/Vis $\left(\mathrm{CHCl}_{3}\right) \lambda_{\max }(E)=457.8(0.22), 488.6(0.60), 525.0 \mathrm{~nm}(1.00)$. - Fluorescence $\left(\mathrm{CHCl}_{3}\right): \lambda_{\max }\left(I_{\text {rel }}\right)=535.0$ (1.00), 578.0 (0.52), $626.5 \mathrm{~nm}$ (0.12). Fluorescence quantum yield $\left(\lambda_{\mathrm{exc}}=489 \mathrm{~nm}, E_{489 \mathrm{~nm} / 1 \mathrm{~nm}}=0.0240\right.$, reference: 2,9-bis-(1-hexyl-heptyl)anthra[2,1,9-def,6,5,10-d'e'f] diisoquinoline-1,3,8,10-tetr aone (1) with $\Phi=1.00$ [40]): 1.00. MS: (DEI $/ 70 \mathrm{eV}): m / z(\%): 722(82)\left[M^{+}\right]$, $679(42)\left[M^{+}-\mathrm{C}_{3} \mathrm{H}_{7}\right], 570(36)\left[M^{+}-\mathrm{C}_{11} \mathrm{H}_{21}\right], 557(58)\left[M^{+}-\mathrm{C}_{12} \mathrm{H}_{23}\right], 418(100)$ $\left[M^{+}-2 \times \mathrm{C}_{11} \mathrm{H}_{21}\right], 404(80)\left[M^{+}-\mathrm{C}_{11} \mathrm{H}_{21}-\mathrm{C}_{12} \mathrm{H}_{23}\right], 391(51)\left[M^{+}-2 \times \mathrm{C}_{12} \mathrm{H}_{23}\right]$. HMRS $\left(\mathrm{C}_{48} \mathrm{H}_{54} \mathrm{~N}_{2} \mathrm{O}_{4}\right)$ : Calcd. $\mathrm{m} / z$ : 722.408, found $\mathrm{m} / z$ : 722.406 .

2-(2,2-Dibutylhept-6-enyl)-9-(2-hydroxyethyl)anthra[2,1,9-def,6,5,10-d'e' $f$ ]diisoquinoline-1,3,8,10-tetraone (21): 9-(2-Hydroxyethyl)2-benzopyrano $\left[6^{\prime}, 5^{\prime} 4^{\prime}: 10,5,6\right]$ anthra[2,1,9-def] isoquinoline-1,3,8,10-tetraone $(600 \mathrm{mg}, 1.38$ mmol),, imidazole ( $5 \mathrm{~g}$ ) and the quantity of a microspatulum of zinc acetate dihydrate under argon atmosphere were melt, stirred at $140^{\circ} \mathrm{C}$, slowly treated with 2,2-dibutylhept-6-enylamine (396 mg, $1.76 \mathrm{mmol}$ ), further stirred at $140^{\circ} \mathrm{C}$ for $3 \mathrm{~h}$, allowed to cool treated with ethanol $(15 \mathrm{~mL})$, precipitated with $2 \mathrm{~N}$ aqueous $\mathrm{HCl}$, collected by vacuum filtration (D4 glass filter), dried in air and purified by column separation $(1000 \mathrm{~mL}$ silica gel, glas column $800 \times 42 \mathrm{~mm}$, chloroform/ethanol 30:1). Yield $742 \mathrm{mg}$ (84\%) reddish brown solid, m.p. > $250^{\circ} \mathrm{C}$. $R_{\mathrm{f}}$-value (silica gel, $\left.\mathrm{CHCl}_{3} / \mathrm{EtOH} 20: 1\right)=0.12$. IR (ATR): $\tilde{v}=3523.4$ $(\mathrm{w}), 3074.3(\mathrm{w}), 2953.5(\mathrm{~m}), 2930.9(\mathrm{~m}), 2862.2(\mathrm{~m}), 1693.5$ (s), 1646.2 (vs), 1593.4 (s), 1577.1 (m), 1506.8 (w), 1438.1 (w), 1403.5 (w), 1336.3 (m), 1251.2 (w), $1169.4(\mathrm{w}), 1126.3(\mathrm{w}), 1059.7(\mathrm{w}), 908.0(\mathrm{w}), 856.9(\mathrm{w}), 808.8(\mathrm{~m}), 793.9$ (w), $744.1 \mathrm{~cm}^{-1}(\mathrm{w}) .{ }^{1} \mathrm{H} \mathrm{NMR}\left(600 \mathrm{MHz}, \mathrm{CDCl}_{3}, 25^{\circ} \mathrm{C}, \mathrm{TMS}\right): \delta=0.89\left(\mathrm{t},{ }^{3}\right.$ Л $\left.\delta, \mathrm{H}\right)$ $\left.=7.0 \mathrm{~Hz}, 6 \mathrm{H}, 2 \times \mathrm{CH}_{3}\right), 1.24-1.33\left(\mathrm{~m}, 14 \mathrm{H}, 7 \times \mathrm{CH}_{2}\right), 1.42-1.47(\mathrm{~m}, 2 \mathrm{H}$, $\left.\mathrm{CH}_{2}\right), 2.01\left(\mathrm{q},{ }^{3} J(\mathrm{H}, \mathrm{H})=7.3 \mathrm{~Hz}, 2 \mathrm{H}, \mathrm{C}_{\mathrm{q}}-\mathrm{CH}_{2}\right), 4.07\left(\mathrm{t},{ }^{3} \mathcal{J}(\mathrm{H}, \mathrm{H})=5.2 \mathrm{~Hz}, 2 \mathrm{H}\right.$, $\left.\mathrm{N}-\mathrm{CH}_{2}-\mathrm{CH}_{2}\right), 4.22\left(\mathrm{~s}, 2 \mathrm{H}, \mathrm{N}-\mathrm{CH}_{2}-\mathrm{C}_{\mathrm{q}}\right), 4.50\left(\mathrm{t},{ }^{3} \mathcal{( H , H}\right)=5.2 \mathrm{~Hz}, 2 \mathrm{H}$, $\left.\mathrm{HO}-\mathrm{CH}_{2}\right), 4.96$ - 4.97 (m, $\left.1 \mathrm{H}, \mathrm{CH}_{2, \text { olefin }}\right), 4.99$ - $5.00\left(\mathrm{~m}, 1 \mathrm{H}, \mathrm{CH}_{2, \text { olefin. }}\right), 5.78$ $5.85\left(\mathrm{~m}, 1 \mathrm{H}, \mathrm{CH}_{\text {olefin. }}\right), 8.42-8.46\left(\mathrm{~m}, 4 \mathrm{H}, 4 \times \mathrm{CH}_{\text {perylene }}\right), 8.52-8.53(\mathrm{~m}, 2 \mathrm{H}, 2 \times$ $\left.\mathrm{CH}_{\text {perylene }}\right), 8.60-8.62 \mathrm{ppm}\left(\mathrm{m}, 2 \mathrm{H}, 2 \times \mathrm{CH}_{\text {prylene }}{ }^{13} \mathrm{C} \mathrm{NMR}\left(150 \mathrm{MHz}, \mathrm{CDCl}_{3}\right.\right.$, $25^{\circ} \mathrm{C}$, TMS): $\delta=14.2,23.1,23.8,25.8,34.8,35.8,35.9,40.7,43.0,45.7,61.5$, $114.3,122.8,122.9,123.2$, 123.6, 129.0, 129.4, 131.2, 131.5, 134.0, 134.8, 139.2, $164.1 \mathrm{ppm}$. UV/Vis $\left(\mathrm{CHCl}_{3}\right): \lambda_{\max }\left(I_{\text {rel }}\right)=458.8(0.22), 490.0(0.60), 526.4 \mathrm{~nm}$ (1.00). Fluorescence $\left(\mathrm{CHCl}_{3}\right): \lambda_{\max }(I)=535.5(1.00), 577.3(0.54), 625.3 \mathrm{~nm}$ (0.13). Fluorescence quantum yield $\left(\lambda_{\text {exc }}=488 \mathrm{~nm}, E_{488 \mathrm{~nm} / \mathrm{lcm}}=0.0344\right.$, reference: 2,9-bis-(1-hexylheptyl)anthra[2,1,9-def;6,5,10-d'e'f] diisoquinoline-1,3,8,10-tetra one (1) with $\Phi=1.00$ [40]): 1.00. MS: $\left(\mathrm{DEI}^{+} / 70 \mathrm{eV}\right): \mathrm{m} / z(\%): 642(100)\left[M^{+}\right]$, $448(64)\left[M^{+}-\mathrm{C}_{14} \mathrm{H}_{27}\right], 433(23)\left[M^{+}-\mathrm{C}_{15} \mathrm{H}_{29}\right], 405(98)\left[M^{+}-\mathrm{C}_{14} \mathrm{H}_{27}-\mathrm{C}_{2} \mathrm{H}_{5} \mathrm{O}\right]$, 390 (13) $\left[M^{+}-\mathrm{C}_{15} \mathrm{H}_{29}-\mathrm{C}_{2} \mathrm{H}_{5} \mathrm{O}\right]$. HMRS $\left(\mathrm{C}_{41} \mathrm{H}_{42} \mathrm{~N}_{2} \mathrm{O}_{5}\right)$ : Calcd. $m / z$. 642.3094; found $\mathrm{m} / \mathrm{z}: 642.3099$.

2-(1-Hexylheptyl)-9-(6-hydroxypentyl)anthra[2,1,9-def,6,5,10-d' $\left.e^{\prime} f\right]$ diis oquinoline-1,3,8,10-tetraone: 9-(1-Hexylheptyl)-2-benzopyrano[6' $6^{\prime} 4^{\prime}: 10,5$, 6] anthra[2,1,9-def]-isoquinoline-1,3,8,10-tetraone $(300 \mathrm{mg}, 0.523 \mathrm{mmol})$, 5-amino-1-pentanol $(80.9 \mathrm{mg}(0.784 \mathrm{mmol})$, imidazole $(3.5 \mathrm{~g})$, the quantity of a 
microspatulum of zincacetate dihydrate, acetic acid $(40 \mathrm{~mL}$, replacement of ethanol) and $2 \mathrm{~N}$ aqueous $\mathrm{HCl}(60 \mathrm{~mL})$ were allowed to react as was described for 2-(2,2-dibutylhept-6-enyl)-9-(2-hydroxy-ethyl)anthra[2,1,9-def6,5,10-d'e'f] diiso quinoline-1,3,8,10-tetraone and purified by colun separation $(800 \mathrm{~mL}$ of silica gel, gas column $780 \times 44 \mathrm{~mm}$, chloroform/ethanol 20:1). Yield $290 \mathrm{mg}(84 \%)$ light red powder, m.p. $>250^{\circ} \mathrm{C}$. $R_{\mathrm{f}}$-value (silica gel, $\left.\mathrm{CHCl}_{3} / \mathrm{EtOH} 20: 1\right)=0.12$. IR $(\mathrm{KBr}): \tilde{v}=3468.1(\mathrm{w}, \mathrm{br}),. 2928.1(\mathrm{w}), 2858.1(\mathrm{w}), 1697.1(\mathrm{~s}), 1657.1$ (vs), $1595.5(\mathrm{~m}), 1578.7(\mathrm{w}), 1507.8(\mathrm{w}), 1439.4(\mathrm{w}), 1404.5(\mathrm{~m}), 1342.5(\mathrm{~m}), 1253.2$ (w), $1176.2(w), 1126.9(w), 1107.9(w), 853.1(w), 810.5(m), 747.5(m), 627.0$ (w), $432.6 \mathrm{~cm}^{-1}$ (w). ${ }^{1} \mathrm{H}$ NMR $\left(600 \mathrm{MHz}, \mathrm{CDCl}_{3}, 25^{\circ} \mathrm{C}, \mathrm{TMS}\right): \delta=0.83\left(\mathrm{t},{ }^{3} \mathcal{J}(\mathrm{H}, \mathrm{H})\right.$ $\left.=7.0 \mathrm{~Hz}, 6 \mathrm{H}, 2 \times \mathrm{CH}_{3}\right), 1.19-1.38\left(\mathrm{~m}, 16 \mathrm{H}, 8 \times \mathrm{CH}_{2}\right), 1.67-1.72(\mathrm{~m}, 2 \mathrm{H}$, $\left.\beta-\mathrm{CH}_{2}\right), 1.80-1.90\left(\mathrm{~m}, 4 \mathrm{H}, 2 \times \mathrm{CH}_{2}\right), 2.22-2.28\left(\mathrm{~m}, 2 \mathrm{H}, \beta-\mathrm{CH}_{2}\right), 3.69(\mathrm{t}$, $\left.{ }^{3}(\mathrm{H}, \mathrm{H})=6.4 \mathrm{~Hz}, 2 \mathrm{H}, \mathrm{N}-\mathrm{CH}_{2}\right), 4.24-4.25\left(\mathrm{~m}, 2 \mathrm{H}, \mathrm{CH}_{2}-\mathrm{OH}\right), 5.16-5.21(\mathrm{~m}, 1$ $\mathrm{H}, \alpha-\mathrm{CH}), 8.59-8.68 \mathrm{ppm}\left(\mathrm{m}, 8 \mathrm{H}, 8 \times \mathrm{CH}_{\text {perylene }}\right) .{ }^{13} \mathrm{C} \mathrm{NMR}\left(150 \mathrm{MHz}, \mathrm{CDCl}_{3}\right.$, $25^{\circ} \mathrm{C}$, TMS): $\delta=14.0,22.6,23.3,26.9,27.9,29.2,31.8,32.4,40.4,54.8,62.8$, $123.0,123.1,123.2,126.4,126.5,129.4,129.6,131.2,131.5,131.9,134.4,134.8$, $163.5 \mathrm{ppm}$. UV/Vis $\left(\mathrm{CHCl}_{3}\right): \lambda_{\max }(\varepsilon)=458.9(17800), 489.9(49000), 526.4 \mathrm{~nm}$ (82000). Fluorescence $\left(\mathrm{CHCl}_{3}\right): \lambda_{\max }(I)=532.9(1.00), 576.3(0.54), 625.3 \mathrm{~nm}$ (0.13). Fluorescence quantum yield $\left(\lambda_{\text {exc }}=489 \mathrm{~nm}, E_{489 \mathrm{~nm} / 1 \mathrm{~cm}}=0.0242\right.$, reference: 2,9-bis-(1-hexylheptyl)anthra[2,1,9-def,6,5,10-d'e'f] diisoquinoline-1,3,8,10-tetra one (1) with $\Phi=1.00$ [40]): 1.00. MS: $\left(\mathrm{DEI}^{+} / 70 \mathrm{eV}\right): \mathrm{m} / z(\%): 658(85)\left[M^{+}\right], 641$ (13) $\left[M^{+}-\mathrm{OH}\right], 573(6)\left[M^{+}-\mathrm{C}_{6} \mathrm{H}_{13}\right], 476(100)\left[M^{+}-\mathrm{C}_{13} \mathrm{H}_{27}\right], 459(11)\left[M^{+}-\right.$ $\mathrm{C}_{13} \mathrm{H}_{27}-\mathrm{OH}$ ], 390 (69) $\left[M^{+}-\mathrm{C}_{13} \mathrm{H}_{27}-\mathrm{C}_{5} \mathrm{H}_{11} \mathrm{O}\right] . \mathrm{C}_{41} \mathrm{H}_{42} \mathrm{~N}_{2} \mathrm{O}_{5}$ (642.3): Calcd. C 76.57, H 7.04, N 4.25; found C 76.31, H 7.06, N 4.07.

2-\{2-(2-Ethoxyethyl)-9-(1-nonyldecyl)anthra[2,1,9-def,6,5,10-d' $e^{\prime} f$ ]diis oquinoline-1,3,8,10-tetraon\}-9-(1-nonyldecyl)anthra[2,1,9-def,6,5,10-d'e'f] diisoquinoline-1,3,8,10-tetraone: 2-(2-Hydroxyethyl)-9-(1-nonyldecyl) anthra [2,1,9-def,6,5,10-d'eff] diisoquinoline-1,3,8,10-tetraone (140 mg, $0.200 \mathrm{mmol}$ ) under argon atmosphere was dissolved in anhydrous chloroform $(5 \mathrm{~mL})$, treated with triethylamine $(61 \mathrm{mg}, 0.60 \mathrm{mmol})$ and then with (2-cyanoethyl) $-N, N$ diisopropylphosphonamidechloride $(122 \mathrm{mg}, 0.553 \mathrm{mmol})$, stirred at room tmperature for $18 \mathrm{~h}$, diluted with chloroform $(120 \mathrm{~mL})$, three times shaken with $5 \%$ aqueous $\mathrm{NaHCO} 3(80 \mathrm{~mL}$ each), dried with magnesium sulphate and purified two times by column separation (1000 mL silica gel, chlorofor/acetone 10:1, glas column $700 \times 55 \mathrm{~mm}$, fraction 4$)$, Yield $30 \mathrm{mg}(22 \%)$ dark red solid, m.p. > $250^{\circ} \mathrm{C} . R_{\mathrm{f}}$-value (silica gel, $\mathrm{CHCl}_{3}$ /acetone 10:1) $=0.06$. IR $(\mathrm{ATR}): \tilde{v}=2921.2$ (vs), 2852.1 (s), 1748.9 (w), 1696.4 (s), 1653.8 (vs), 1592.9 (s), 1577.0 (m), 1506.6 $(\mathrm{w}), 1436.0(\mathrm{w}), 1403.6(\mathrm{~m}), 1338.8(\mathrm{~s}), 1247.4(\mathrm{~m}), 1194.0(\mathrm{w}), 1175.5(\mathrm{w})$, $1125.5(\mathrm{w}), 1065.1(\mathrm{w}), 1005.6(\mathrm{w}), 851.1(\mathrm{w}), 808.5(\mathrm{~m}), 788.1(\mathrm{w}), 744.0 \mathrm{~cm}^{-1}$ (m). ${ }^{1} \mathrm{H}$ NMR (600 MHz, $\mathrm{CDCl}_{3}, 25^{\circ} \mathrm{C}$, TMS): $\delta=0.83\left(\mathrm{t},{ }^{3} / \mathrm{H}, \mathrm{H}\right)=7.0 \mathrm{~Hz}, 12$ $\left.\mathrm{H}, 4 \times \mathrm{CH}_{3}\right), 1.22-1.41\left(\mathrm{~m}, 56 \mathrm{H}, 28 \mathrm{CH}_{2}\right), 1.87-1.95\left(\mathrm{~m}, 4 \mathrm{H}, \beta-\mathrm{CH}_{2}\right), 2.20$ $\left.2.29\left(\mathrm{~m}, 4 \mathrm{H}, \beta-\mathrm{CH}_{2}\right), 4.53\left(\mathrm{t},{ }^{3}\right)(\mathrm{H}, \mathrm{H})=5.1 \mathrm{~Hz}, 4 \mathrm{H}, 2 \times \mathrm{N}-\mathrm{CH}_{2}-\mathrm{CH}_{2}\right), 4.62(\mathrm{t}$, $\left.{ }^{3} /(\mathrm{H}, \mathrm{H})=5.2 \mathrm{~Hz}, 4 \mathrm{H}, 2 \times \mathrm{CH}_{2} \mathrm{O}\right), 5.12-5.19(\mathrm{~m}, 2 \mathrm{H}, 2 \times \alpha-\mathrm{CH}), 8.00-8.02(\mathrm{~m}$, 
$\left.2 \mathrm{H}, 2 \times \mathrm{CH}_{\text {perylene }}\right), 8.11-8.13\left(\mathrm{~m}, 2 \mathrm{H}, 2 \times \mathrm{CH}_{\text {perylene }}\right), 8.24-8.26(\mathrm{~m}, 2 \mathrm{H}, 2 \times$ $\left.\mathrm{CH}_{\text {perylene }}\right), 8.39-8.45 \mathrm{ppm}\left(\mathrm{m}, 2 \mathrm{H}, 2 \times \mathrm{CH}_{\text {perylene }}\right) .{ }^{13} \mathrm{C} \mathrm{NMR}\left(150 \mathrm{MHz}, \mathrm{CDCl}_{3}\right.$, $25^{\circ} \mathrm{C}$, TMS): $\delta=14.1,22.7,27.1,29.3,29.6,31.9,32.3,39.1,54.9,65.0,122.4$, $122.5,122.7,125.7,128.9,129.0,131.0,133.5,134.1,163.0 \mathrm{ppm}$. UV/Vis $\left(\mathrm{CHCl}_{3}\right)$ : $\lambda_{\max }(E)=459.0(0.23), 490.0(0.63), 527.0 \mathrm{~nm}(1.00)$. Fluorescence $\left(\mathrm{CHCl}_{3}\right): \lambda_{\max }$ $(I)=573.8$ (1.00), $577.0(0.52), 625.3 \mathrm{~nm}$ (0.13). Fluorescence quantum yield ( $\lambda_{\text {exc }}=488 \mathrm{~nm}, E_{488 \mathrm{~nm} / 1 \mathrm{~cm}}=0.0271$, reference: 2,9-bis-(1-hexylheptyl)anthra[2,1, 9-def,6,5,10- d'e'f] diisoquinoline-1,3,8,10-tetraone (1) with $\Phi=1.00$ [40]): 1.00 . MS: $\left(\mathrm{DEI}^{+} / 70 \mathrm{eV}\right): \mathrm{m} / z(\%): 1383(<1)\left[M^{+}\right], 1366(<1)\left[M^{+}-\mathrm{HO}\right], 1257(<1)$ $\left[M^{+}-\mathrm{C}_{9} \mathrm{H}_{19}\right], 1100(<1)\left[M^{+}-\mathrm{C}_{9} \mathrm{H}_{19}-\mathrm{OH}\right], 700(64)\left[M^{+}-\mathrm{C}_{45} \mathrm{H}_{51} \mathrm{~N}_{2} \mathrm{O}_{4}\right], 684$ (39) $\left[M^{+}-\mathrm{C}_{45} \mathrm{H}_{51} \mathrm{~N}_{2} \mathrm{O}_{5}\right], 432$ (4) $\left[M^{+}-\mathrm{C}_{45} \mathrm{H}_{51} \mathrm{~N}_{2} \mathrm{O}_{4}-\mathrm{C}_{9} \mathrm{H}_{19}\right], 418$ (74) $\left[M^{+}-\right.$ $\mathrm{C}_{45} \mathrm{H}_{51} \mathrm{~N}_{2} \mathrm{O}_{5}-\mathrm{C}_{9} \mathrm{H}_{19}$.

2-(2-Bromethyl)-9-(1-nonyldecyl)anthra[2,1,9-def,6,5,10-d'e'f] diisoquin oline-1,3,8,10-tetraone: 2-(2-Hydroxyethyl)-9-(1-nonyldecyl)anthra[2,1,9-def; $6,5,10-d^{\prime} e^{\prime} f$ ] diiso-quinoline-1,3,8,10-tetraone (100 $\left.\mathrm{mg}, 0.143 \mathrm{mmol}\right)$ was dissolved in chloroform $(6 \mathrm{~mL})$, treated with phosphorustribromide (193 mg, $0.713 \mathrm{mmol}$ ), heated to reflux with stirring for $4 \mathrm{~h}$, cooled to $0^{\circ} \mathrm{C}$, precipitated with methanol (25 mL), collected by vacuun filtration (D4 micro glass filter) and purified by column separation $(500 \mathrm{~mL}$ silica gel, chloroform/ethylacetate $30: 1$, glass column $500 \times 44 \mathrm{~mm}$, second fraction). Yield $30 \mathrm{mg}(27 \%)$ dark red solid, m.p. $>250{ }^{\circ} \mathrm{C} . R_{\mathrm{f}}$-value (silica gel, $\mathrm{CHCl}_{3} /$ EtOAc $\left.30: 1\right)=0.64 .{ }^{1} \mathrm{H} \mathrm{NMR}(600$ $\left.\mathrm{MHz}, \mathrm{CDCl}_{3}, 25^{\circ} \mathrm{C}, \mathrm{TMS}\right): \delta=0.82\left(\mathrm{t},{ }^{3} \mathcal{J}(\mathrm{H}, \mathrm{H})=6.4 \mathrm{~Hz}, 6 \mathrm{H}, 2 \times \mathrm{CH}_{3}\right), 1.18-$ $1.36\left(\mathrm{~m}, 28 \mathrm{H}, 14 \times \mathrm{CH}_{2}\right), 1.81-1.97\left(\mathrm{~m}, 2 \mathrm{H}, \beta-\mathrm{CH}_{2}\right), 2.17-3.71(\mathrm{~m}, 2 \mathrm{H}$, $\left.\beta-\mathrm{CH}_{2}\right), 3.71\left(\mathrm{t},{ }^{3} \mathcal{J}(\mathrm{H}, \mathrm{H})=7.0 \mathrm{~Hz}, 2 \mathrm{H}, \mathrm{CH}_{2}-\mathrm{Br}\right), 4.61\left(\mathrm{t},{ }^{3} \mathcal{J}(\mathrm{H}, \mathrm{H})=7.0 \mathrm{~Hz}, 2 \mathrm{H}\right.$, $\left.\mathrm{N}-\mathrm{CH}_{2}\right), 5.18(\mathrm{~m}, 1 \mathrm{H}, \mathrm{CH}), 8.40-8.62 \mathrm{ppm}\left(\mathrm{m}, 8 \mathrm{H}, 8 \times \mathrm{CH}_{\text {perylene }}\right)$.

2-Amino-9-(2-but-3-enyl-2-octyldecyl)-anthra[2,1,9-def,6,5,10-d'e'f']diis oquinoline-1,3,8,10-tetraone: 9-Amino-2-benzopyrano- $\left[6^{\prime}, 5^{\prime} 4^{\prime}: 10,5,6\right]$ anthra [2,1,9-def] iso-quinoline-1,3,8,10-tetraone (700 mg, $1.72 \mathrm{mmol}$ ), 2-but-3-enyl-2octyldecylamine $(5 \mathrm{c}, 836 \mathrm{mg}, 2.58 \mathrm{mmol})$, imidazole $(4.0 \mathrm{~g})$, the quantity of a micro spatulum of zincacetate dihydrate, ethanol $(15 \mathrm{~mL})$ and $2 \mathrm{~N}$ aqueous $\mathrm{HCl}$ $(100 \mathrm{~mL})$ were allowed to react analogously to 2-(2,2-dibutylhept-6-enyl)-9(2-hydroxyethyl)anthra[2,1,9-def,6,5,10-d'e'f] diisoquinoline-1,3,8,10-tetraone, dried in vacuo with calciumchloride and then with phosphorous(V)oxid and purified by column separation ( $800 \mathrm{~mL}$ silica gel, chloroform/ethanol 30:1, glass column $780 \times 44 \mathrm{~mm})$. Yield $579 \mathrm{mg}(47 \%)$ dark red solid, m.p. $>250^{\circ} \mathrm{C}$. $R_{\mathrm{f}}$-value (silica gel, $\left.\mathrm{CHCl}_{3} / \mathrm{EtOH} 30: 1\right)=0.10$. IR (ATR): $\tilde{v}=3329.9(\mathrm{w})$, 3243.5 (w), 3073.7 (w), 2921.7 (vs), 2851.6 (s), 1696.7 (vs), 1652.1 (vs), 1592.6 (vs), $1576.3(\mathrm{~m}), 1507.3(\mathrm{w}), 1456.9(\mathrm{w}), 1436.0(\mathrm{w}), 1402.3(\mathrm{~m}), 1367.5(\mathrm{~m})$, $1339.7(\mathrm{~s}), 1301.1(\mathrm{~m}), 1251.2(\mathrm{~m}), 1201.2(\mathrm{w}), 1171.2(\mathrm{w}), 1123.9(\mathrm{w}), 991.0(\mathrm{w})$, $903.3(\mathrm{w}), 850.1(\mathrm{w}), 807.9(\mathrm{~m}), 793.9(\mathrm{w}), 756.9(\mathrm{w}), 737.8(\mathrm{~m}), 666.6 \mathrm{~cm}^{-1}(\mathrm{w})$. ${ }^{1} \mathrm{H}$ NMR (600 MHz, $\mathrm{CDCl}_{3}, 25^{\circ} \mathrm{C}$, TMS): $\delta=0.86\left(\mathrm{t},{ }^{3} \mathcal{J}(\mathrm{H}, \mathrm{H})=6.9 \mathrm{~Hz}, 6 \mathrm{H}, 2 \times\right.$ $\left.\mathrm{CH}_{3}\right), 1.24-1.40\left(\mathrm{~m}, 30 \mathrm{H}, 15 \times \mathrm{CH}_{2}\right), 2.10-2.14\left(\mathrm{~m}, 2 \mathrm{H}, \mathrm{CH}_{2}\right), 4.24(\mathrm{~s}, 2 \mathrm{H}$, $\left.\mathrm{N}-\mathrm{CH}_{2}\right), 4.90$ - 4.91 (m, $\left.1 \mathrm{H}, \mathrm{CH}_{2, \text { olefin. }}\right), 4.99$ - $5.02\left(\mathrm{~m}, 1 \mathrm{H}, \mathrm{CH}_{2, \text { olefin }}\right), 5.79$ - 5.85 
(m, $\left.1 \mathrm{H}, \mathrm{CH}_{\text {olefin. }}\right), 8.51-8.64 \mathrm{ppm}\left(\mathrm{m}, 8 \mathrm{H}, 8 \times \mathrm{CH}_{\text {perylene }}\right) .{ }^{13} \mathrm{C} \mathrm{NMR}(150 \mathrm{MHz}$, $\mathrm{CDCl}_{3}, 25^{\circ} \mathrm{C}$, TMS): $\delta=14.2,22.7,23.6,28.2,29.4,29.7,30.8,32.0,35.6,36.3$, $40.8,45.7,113.9,122.3,122.9,123.4,123.8,126.0,127.9,129.1,131.4,131.6$, 134.0, 135.1, 139.8, 160.1, $164.1 \mathrm{ppm}$. UV/Vis $\left(\mathrm{CHCl}_{3}\right): \lambda_{\max }(E)=460(0.24)$, 491.6 (0.62), $528.4 \mathrm{~nm}$ (1.00). Fluorescence $\left(\mathrm{CHCl}_{3}\right)$ : No emission detected. MS: $\left(\mathrm{DEI}^{+} / 70 \mathrm{eV}\right): m / z(\%): 711(21)\left[M^{+}\right], 696(13)\left[M^{+}-\mathrm{NH}_{2}\right], 419(100)\left[M^{+}-\right.$ $\left.\mathrm{C}_{21} \mathrm{H}_{41}\right], 404$ (76) $\left[M^{+}-\mathrm{C}_{22} \mathrm{H}_{43}\right], 390(28)\left[M^{+}-\mathrm{C}_{22} \mathrm{H}_{43}-\mathrm{NH}_{2}\right]$. HMRS $\left(\mathrm{C}_{46} \mathrm{H}_{54} \mathrm{~N}_{3} \mathrm{O}_{4}\right)$ : Calcd. $\mathrm{m} / \mathrm{z}: 712.411\left[M^{+}+\mathrm{H}\right]$; found $\mathrm{m} / \mathrm{z}: 712.410$.

2-Allyl-9-(1-hexylheptyl)anthra[2,1,9-def,6,5,10-d'e'f] diisoquinoline-1,3, 8,10-tetraon(3): 9-(1-Hexylheptyl)-2-benzopyrano $\left[6^{\prime}, 5^{\prime} 4^{\prime}: 10,5,6\right]$ anthra[2,1, 9-def] isoquinoline-1,3,8,10-tetraone $(500 \mathrm{mg}, 0.872 \mathrm{mmol}$ ) the quantity of a microspatulum of zincacetate dihydrate, acetic acid $(80 \mathrm{~mL}$, replacement of ethanol), imidazole $(8.0 \mathrm{~g})$, allylamine $(65.0 \mathrm{mg}, 1.14 \mathrm{mmol})$ and $2 \mathrm{~N}$ aqueous $\mathrm{HCl}(100 \mathrm{~mL})$ were allowed to react as was described for 2-(2,2-dibutylhept6-enyl)-9-(2-hydroxyethyl)anthra[2,1,9-def,6,5,10-d'e'f] diisoquinoline-1,3,8,10-t etraone and purified by column separation $(800 \mathrm{~mL}$ of silica gel, chloroform/ ethanol 30:1, glas column $780 \times 44 \mathrm{~mm})$. Yield $405 \mathrm{mg}(76 \%)$ light red solid, m.p. $>250^{\circ} \mathrm{C}$. $R_{\mathrm{f}}$-value (silica gel, $\left.\mathrm{CHCl}_{3} / \mathrm{EtOH} 30: 1\right)=0.63$. IR $(\mathrm{KBr}): \tilde{v}=$ $2954.6(\mathrm{~m}), 2926.7$ (s), $2856.6(\mathrm{~m}), 1770.3(\mathrm{w}), 1698.2$ (s), 1659.2 (s), 1595.1 (s), $1578.5(\mathrm{~m}), 1506.7(\mathrm{w}), 1482.2(\mathrm{w}), 1456.2(\mathrm{w}), 1436.1(\mathrm{~m}), 1404.4(\mathrm{~m}), 1376.1$ (m), 1345.4 (s), 1251.7 (m), 1216.0 (w), 1193.9 (w), 1174.7 (m), 1156.3 (w), $1125.9(\mathrm{w}), 1106.7(\mathrm{w}), 997.7(\mathrm{w}), 930.5(\mathrm{w}), 852.0(\mathrm{w}), 810.2(\mathrm{~m}), 796.2(\mathrm{w})$, $786.2(\mathrm{w}), 747.9(\mathrm{~m}), 723.9 \mathrm{~cm}^{-1}(\mathrm{w}) .{ }^{1} \mathrm{H}$ NMR (600 MHz, $\left.\mathrm{CDCl}_{3}, 25^{\circ} \mathrm{C}, \mathrm{TMS}\right): \delta$ $\left.=0.83\left(\mathrm{t},{ }^{3}\right)(\mathrm{H}, \mathrm{H})=7.0 \mathrm{~Hz}, 6 \mathrm{H}, 2 \times \mathrm{CH}_{3}\right), 1.21-1.37\left(\mathrm{~m}, 16 \mathrm{H}, 8 \times \mathrm{CH}_{2}\right), 1.88$ $\left(\mathrm{m}, 2 \mathrm{H}, \alpha-\mathrm{CH}_{2}\right), 2.25\left(\mathrm{~m}, 2 \mathrm{H}, \alpha-\mathrm{CH}_{2}\right), 4.83\left(\mathrm{~d}, 2 \mathrm{H},{ }^{3} \mathcal{J}(\mathrm{H}, \mathrm{H})=5.9 \mathrm{~Hz}, \mathrm{~N}-\mathrm{CH}_{2}\right)$, 5.19 (m, $1 \mathrm{H}, \mathrm{N}-\mathrm{CH}), 5.25$ (m, $1 \mathrm{H}, \mathrm{CH}_{2 \text {,olefin. }}$ ), 5.37 (m, $1 \mathrm{H}, \mathrm{CH}_{2 \text {,olefin. }}$ ), 6.02 (m, 1 $\left.\mathrm{H}, \mathrm{CH}_{\text {olefin }}\right), 8.55-8.66 \mathrm{ppm}\left(\mathrm{m}, 8 \mathrm{H}, 8 \times \mathrm{CH}_{\text {perylene }}\right) .{ }^{13} \mathrm{C} \mathrm{NMR}\left(151 \mathrm{MHz}, \mathrm{CDCl}_{3}\right.$, $25^{\circ} \mathrm{C}$, TMS): $\delta=16.0,24.6,28.9,31.2,33.7,34.4,44.6,56.8,120.0,124.9,125.1$, $128.3,128.5,131.5,133.1,133.5,133.9,136.3,136.8,165.1 \mathrm{ppm}$. UV/Vis $\left(\mathrm{CHCl}_{3}\right)$ : $\lambda_{\max }(E)=458(0.22), 489(0.60), 526 \mathrm{~nm}(1.00)$. Fluorescence $\left(\mathrm{CHCl}_{3}\right): \lambda_{\max }(I)=$ 535 (1.00), $579 \mathrm{~nm}$ (0.41). Fluorescence quantum yield $\left(\mathrm{CHCl}_{3}, \lambda_{\mathrm{exc}}=488 \mathrm{~nm}\right.$, $E_{488 \mathrm{~nm} / 1 \mathrm{~cm}}=0.0285$; reference: 2,9-bis-(1-hexylheptyl)anthra-[2,1,9-def;6,5,10$d^{\prime} e^{\prime} f$ ] diisoquinoline-1,3,8,10-tetraone (1) with $\Phi=1.00$ [40]): 1.00. $\mathrm{MS}_{\text {(DEI }} / 70$ $\mathrm{eV}): m / z(\%): 612(62)\left[M^{+}\right], 430(100)\left[M^{+}-\mathrm{C}_{13} \mathrm{H}_{26}\right], 415(67)\left[430-\mathrm{CH}_{3}\right], 390$ (3) $\left[M^{+}-\mathrm{C}_{13} \mathrm{H}_{26}-\mathrm{C}_{3} \mathrm{H}_{4}\right]$. HMRS $\left(\mathrm{C}_{40} \mathrm{H}_{40} \mathrm{~N}_{2} \mathrm{O}_{4}\right)$ : Calcd. $\mathrm{m} / z$ : 612.299; found $\mathrm{m} / z$. 612.300 .

2-(2,2-Dibutylhex-5-enyl)-9-(1-hexylheptyl)anthra[2,1,9-def,6,5,10-d'e'f] diisoquinoline-1,3,8,10-tetraone (8): 9-(1-Hexylheptyl)-2-benzopyrano[6' ${ }^{\prime} 5^{\prime} 4^{\prime}$ : $10,5,6]$ anthra[2,1,9def] isoquinoline-1,3,8,10-tetraone (2, $100 \mathrm{mg}, 0.174 \mathrm{mmol})$ 2,2-dibutylhex-5-enylamine ( $80.0 \mathrm{mg}, 0.348 \mathrm{mmol})$, imidazole $(2.70 \mathrm{~g})$, the quantity of a microspatulum of zincacetate dihydrate, ethanol $(10 \mathrm{~mL})$ and $2 \mathrm{~N}$ aqueous $\mathrm{HCl}(50 \mathrm{~mL})$ were allowed to react as was described for 2-(2,2dibutylhept-6-enyl)-9-(2-hydroxyethyl)anthra[2,1,9-def,6,5,10-d'e'f] diisoquinoli 
ne-1,3,8,10-tetraone and purified by medium pressure chromatography (silica gel, chloroform $25 \mathrm{~mL} \cdot \mathrm{min}^{-1}$, column $36 \times 460 \mathrm{~mm}$ ). Yield $869 \mathrm{mg}$ (94\%) light red powder, m.p. $>250^{\circ} \mathrm{C} . R_{\mathrm{f}}$-value (silica gel, $\left.\mathrm{CHCl}_{3}\right)=0.39$. IR $(\mathrm{KBr}): \tilde{v}=$ 3436.5 (m, br.), 2955.5 (s), 2928.9 (s), 2858.8 (m), 1698.8 (vs), 1659.5 (vs), 1595.2 (vs), $1578.5(\mathrm{~m}), 1506.2(\mathrm{w}), 1456.4(\mathrm{w}), 1436.5(\mathrm{w}), 1405.7(\mathrm{~m}), 1337.4(\mathrm{vs})$, $1252.5(\mathrm{~m}), 1213.4(\mathrm{w}), 1175.3(\mathrm{w}), 1125.2(\mathrm{w}), 1106.8(\mathrm{w}), 995.8(\mathrm{w}), 908.8(\mathrm{w})$, $852.2(\mathrm{w}), 810.6(\mathrm{~m}), 748.4 \mathrm{~cm}^{-1}(\mathrm{~m}) .{ }^{1} \mathrm{H}$ NMR (600 Mhz, $\left.\mathrm{CDCl}_{3}, 25^{\circ} \mathrm{C}, \mathrm{TMS}\right): \delta$ $\left.=0.83\left(\mathrm{t},{ }^{3}\right)(\mathrm{H}, \mathrm{H})=7.0 \mathrm{~Hz}, 6 \mathrm{H}, 2 \times \mathrm{CH}_{3}\right), 0.89\left(\mathrm{t},{ }^{3}\right)(\mathrm{H}, \mathrm{H})=7.0 \mathrm{~Hz}, 6 \mathrm{H}, 2 \times$ $\left.\mathrm{CH}_{3}\right), 1.19$ - $1.38\left(\mathrm{~m}, 28 \mathrm{H}, 14 \times \mathrm{CH}_{2}\right), 1.38-1.41\left(\mathrm{~m}, 2 \mathrm{H}, \mathrm{CH}_{2}\right), 1.84-1.90(\mathrm{~m}, 2$ $\left.\mathrm{H}, \beta-\mathrm{CH}_{2}\right), 2.10-2.14\left(\mathrm{~m}, 2 \mathrm{H}, \mathrm{CH}_{2}\right), 2.23-2.28\left(\mathrm{~m}, 2 \mathrm{H}, \beta-\mathrm{CH}_{2}\right), 4.25(\mathrm{~s}, 2 \mathrm{H}$, $\left.\mathrm{CH}_{2}\right), 4.89-4.91\left(\mathrm{~m}, 1 \mathrm{H}, \mathrm{CH}_{2, \text { olefin }}\right), 4.99-5.02\left(\mathrm{~m}, 1 \mathrm{H}, \mathrm{CH}_{2 \text {, olefin. }}\right), 5.16-5.21$ $(\mathrm{m}, 1 \mathrm{H}, \alpha-\mathrm{CH}), 5.79-5.86\left(\mathrm{~m}, 1 \mathrm{H}, \mathrm{CH}_{\text {olefin. }}\right), 8.61-8.69 \mathrm{ppm}(\mathrm{m}, 8 \mathrm{H}, 8 \times$ $\mathrm{CH}_{\text {perylene }}$ ). ${ }^{13} \mathrm{C}$ NMR (151 Mhz, $\mathrm{CDCl}_{3}, 25^{\circ} \mathrm{C}$, TMS): $\delta=15.4,15.5,23.9,25.1$, 27.1, 28.3, 29.5, 30.6, 33.1, 33.7, 36.8, 37.2, 42.0, 46.8, 56.1, 115.2, 124.2, 124.9, $127.9,130.7,131.0,132.5,133.3,136.0,141.1,165.7 \mathrm{ppm} . \mathrm{UV} / \mathrm{Vis}\left(\mathrm{CHCl}_{3}\right): \lambda_{\max }$ $(E)=458.2(0.22), 489.0(0.60), 525.4 \mathrm{~nm}(1.00)$. Fluorescence $\left(\mathrm{CHCl}_{3}\right): \lambda_{\max }(I)=$ $532.2(1.00), 576.5(0.35) \mathrm{nm}$. Fluorescence quantum yield $\left(\lambda_{\text {exc }}=487 \mathrm{~nm}, E_{487}\right.$ $\mathrm{nm} / 1 \mathrm{~cm}=0.0440$, reference: 2,9-bis-(1-hexylheptyl)anthra[2,1,9-def;6,5,10-d'eff]-

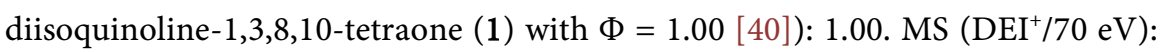
$m / z$ (\%): 766 (45) $\left[M^{+}\right], 586(30)\left[M^{+}-\mathrm{C}_{12} \mathrm{H}_{24}-\mathrm{CH}_{2}\right], 404(100)\left[M^{+}-\mathrm{C}_{26} \mathrm{H}_{50}\right]$, 390 (49) [404- $\left.\mathrm{CH}_{2}\right]$. HMRS $\left(\mathrm{C}_{51} \mathrm{H}_{63} \mathrm{~N}_{2} \mathrm{O}_{4}\right)$ : Calcd. $m / z$. $767.478\left[M^{+}+\mathrm{H}\right]$; found $\mathrm{m} / \mathrm{z} .767 .478$.

2-(2-But-3-enyl-2-hexyloctyl)-9-(1-hexylheptyl)anthra[2,1,9-def,6,5,10- $d^{\prime}$ $\left.e^{\prime} f\right]$ diisoquinoline-1,3,8,10-tetraone: 9-(1-Hexylheptyl)-2-benzopyrano[6', $5^{\prime} 4^{\prime}$ : 10,5,6] anthra[2,1,9-def] isoquinoline-1,3,8,10-tetraone (107 $\mathrm{mg}, 0.187 \mathrm{mmol}$ ), 2-but-3-enyl-2-hexyloctyl-amine (100 $\mathrm{mg}, 0.373 \mathrm{mmol})$, imidazole $(2.5 \mathrm{~g})$, ethanol, $(10 \mathrm{~mL})$ and $2 \mathrm{~N}$ aqueous $\mathrm{HCl}(75 \mathrm{~mL})$ were allowed to react as was described for 2-(2,2-dibutylhept-6-enyl)-9-(2-hydroxyethyl)anthra[2,1,9-def,6,5, 10-d'e'f] diisoquinoline-1,3,8,10-tetraone and purified by column separation (750 mL of silica gel, chloroform and then $350 \mathrm{~mL}$ of silica gel, dichloromethane, column $600 \times 34 \mathrm{~mm})$. Yield $109 \mathrm{mg}(76 \%)$ light red solid, m.p. $>250^{\circ} \mathrm{C}$.

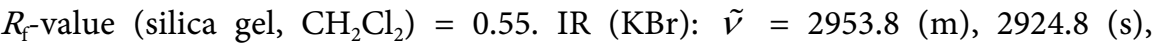
$2854.4(\mathrm{~m}), 1696.1(\mathrm{~s}), 1652.1(\mathrm{vs}), 1594.2(\mathrm{~s}), 1578.1(\mathrm{~m}), 1507.0(\mathrm{w}), 1456.6(\mathrm{w})$, $1434.9(\mathrm{w}), 1404.8(\mathrm{~m}), 1333.9$ (s), $1252.6(\mathrm{~m}), 1211.7(\mathrm{w}), 1174.9(\mathrm{w}), 1123.7$ (w), $1107.5(\mathrm{w}), 993.0(\mathrm{w}), 904.8(\mathrm{w}), 850.7(\mathrm{w}), 808.0(\mathrm{~m}), 745.8(\mathrm{~m}), 669.8 \mathrm{~cm}^{-1}$ (w). ${ }^{1} \mathrm{H} \mathrm{NMR}\left(600 \mathrm{MHz}, \mathrm{CDCl}_{3}, 25^{\circ} \mathrm{C}, \mathrm{TMS}\right): \delta=0.83\left(\mathrm{t},{ }^{3}\right)(\mathrm{H}, \mathrm{H})=7.0 \mathrm{~Hz}, 6 \mathrm{H}$, $\left.\left.2 \times \mathrm{CH}_{3}\right), 0.87\left(\mathrm{t},{ }^{3}\right)(\mathrm{H}, \mathrm{H})=6.8 \mathrm{~Hz}, 6 \mathrm{H}, 2 \times \mathrm{CH}_{3}\right), 1.20-1.36(\mathrm{~m}, 36 \mathrm{H}, 18 \times$ $\left.\mathrm{CH}_{2}\right), 1.38$ - $1.41\left(\mathrm{~m}, 2 \mathrm{H}, \mathrm{CH}_{2}\right), 1.84-1.89\left(\mathrm{~m}, 2 \mathrm{H}, \beta-\mathrm{CH}_{2}\right), 2.10-2.14(\mathrm{~m}, 2 \mathrm{H}$, $\left.\mathrm{C}_{\mathrm{q}}-\mathrm{CH}_{2}\right), 2.22-2.28\left(\mathrm{~m}, 2 \mathrm{H}, \beta-\mathrm{CH}_{2}\right), 4.25\left(\mathrm{~s}, 2 \mathrm{H}, \mathrm{N}-\mathrm{CH}_{2}-\mathrm{C}_{\mathrm{q}}\right), 4.89-4.92(\mathrm{~m}, 1$ $\left.\mathrm{H}, \mathrm{CH}_{2, \text { lefinin }}\right), 4.98-5.02\left(\mathrm{~m}, 1 \mathrm{H}, \mathrm{CH}_{2, \text { lefin. }}\right), 5.16-5.21(\mathrm{~m}, 1 \mathrm{H}, \alpha-\mathrm{CH}), 5.79$ $5.86\left(\mathrm{~m}, 1 \mathrm{H}, \mathrm{CH}_{\text {olefin. }}\right), 8.63-8.69 \mathrm{ppm}\left(\mathrm{m}, 8 \mathrm{H}, 8 \times \mathrm{CH}_{\text {perylene }}\right) .{ }^{13} \mathrm{C} \mathrm{NMR}(150$ $\mathrm{MHz}, \mathrm{CDCl}_{3}, 25^{\circ} \mathrm{C}$, TMS): $\delta=14.0,22.6,22.7,23.5,26.9,29.2,30.4,31.8,31.9$, $32.4,35.6,36.2,40.8,54.8,113.8,123.4,123.5,126.5,129.4,129.7,131.5,134.7$, 
139.8, 164.4 ppm. UV/Vis $\left(\mathrm{CHCl}_{3}\right): \lambda_{\max }(E)=458.0(0.22), 489.4(0.60), 525.8$ $\mathrm{nm}(1.00)$. Fluorescence $\left(\mathrm{CHCl}_{3}\right): \lambda_{\max }(I)=534.0(1.00), 576.0(0.50), 624.8 \mathrm{~nm}$ (0.11). Fluorescence quantum yield $\left(\lambda_{\text {exc }}=488 \mathrm{~nm}, E_{488 \mathrm{~nm} / 1 \mathrm{~cm}}=0.0282\right.$, reference: 2,9-bis-(1-hexylheptyl)anthra[2,1,9-def,6,5,10-d'e'f] diisoquinoline-1,3,8,10tetraone (1) with $\Phi=1.00$ [40]): 1.00. MS: $\left(\mathrm{DEI}^{+} / 70 \mathrm{eV}\right): \mathrm{m} / z(\%): 822(86)\left[M^{+}\right]$, $767(21)\left[M^{+}-\mathrm{C}_{4} \mathrm{H}_{7}\right], 586(35)\left[M^{+}-\mathrm{C}_{17} \mathrm{H}_{33}\right], 573(16)\left[M^{+}-\mathrm{C}_{18} \mathrm{H}_{35}\right], 404$ (100) $\left[M^{+}-\mathrm{C}_{13} \mathrm{H}_{27}-\mathrm{C}_{17} \mathrm{H}_{33}\right], 391(27)\left[M^{+}-\mathrm{C}_{13} \mathrm{H}_{27}-\mathrm{C}_{18} \mathrm{H}_{35}\right]$. HMRS $\left(\mathrm{C}_{55} \mathrm{H}_{71} \mathrm{~N}_{2} \mathrm{O}_{4}\right)$ : Calcd. $\mathrm{m} / z$ : $823.541\left[M^{+}+\mathrm{H}\right]$; found $\mathrm{m} / z .823 .547$.

2-(1-Hexylheptyl)-9-(2,2-dibutylhept-6-enyl)anthra[2,1,9-def6,5,10-d'e'f' ]diisoquinoline-1,3,8,10-tetraone (10): 9-(1-Hexylheptyl)-2-benzopyrano-[6', 5' 4': 10,5,6] anthra[2,1,9-def] isoquinoline-1,3,8,10-tetraone (103 mg, $180 \mu \mathrm{mol})$ 2,2-dibutylhept-6-enylamine (53 $\mathrm{mg}, 0.23 \mathrm{mmol})$, imidazole (2.7 g) ethanol (10 $\mathrm{mL})$ and $2 \mathrm{~N}$ aqueous $\mathrm{HCl}(50 \mathrm{~mL})$ were allowed to react as was described for 2-(2,2-dibutylhept-6-enyl)-9-(2-hydroxy-ethyl)anthra[2,1,9-def,6,5,10-d'e'f]diiso quinoline-1,3,8,10-tetraone and purified by column separation $(500 \mathrm{~mL}$ of silica gel, chloroform, column $500 \times 44 \mathrm{~mm})$. Yield $121 \mathrm{mg}(86 \%)$ light red, shiny solid, m.p. $>250^{\circ} \mathrm{C} . R_{\mathrm{f}}$-value (silica gel, $\left.\mathrm{CHCl}_{3}\right)=0.37$. $\mathrm{IR}(\mathrm{KBr}): \tilde{v}=2954.0$ (m), 2926.7 (m), 2856.9 (m), 1696.5 (s), 1652.7 (vs), 1594.5 (m), 1578.1 (w), $1507.3(\mathrm{w}), 1456.9(\mathrm{w}), 1435.5(\mathrm{w}), 1405.2(\mathrm{w}), 1334.8(\mathrm{~s}), 1253.6(\mathrm{~m}), 1212.1$ $(\mathrm{w}), 1175.8(\mathrm{w}), 1123.9(\mathrm{w}), 1107.0(\mathrm{w}), 996.8(\mathrm{w}), 906.2(\mathrm{w}), 850.8(\mathrm{w}), 808.3$ (m), $746.3(\mathrm{~m}), 669.7 \mathrm{~cm}^{-1}(\mathrm{w}) .{ }^{1} \mathrm{H}$ NMR $\left(600 \mathrm{MHz}, \mathrm{CDCl}_{3}, 25^{\circ} \mathrm{C}, \mathrm{TMS}\right): \delta=0.83$ $\left(\mathrm{t},{ }^{3} \mathcal{J}(\mathrm{H}, \mathrm{H})=6.8 \mathrm{~Hz}, 6 \mathrm{H}, 2 \times \mathrm{CH}_{3}\right), 0.89\left(\mathrm{t},{ }^{3} \mathcal{J}(\mathrm{H}, \mathrm{H})=6.8 \mathrm{~Hz}, 6 \mathrm{H}, 2 \times \mathrm{CH}_{3}\right), 1.22$ - $1.36\left(\mathrm{~m}, 30 \mathrm{H}, 15 \times \mathrm{CH}_{2}\right), 1.41-1.49\left(\mathrm{~m}, 2 \mathrm{H}, \mathrm{CH}_{2}\right), 1.82-1.91\left(\mathrm{~m}, 2 \mathrm{H}, \mathrm{CH}_{2}\right)$, 1.99 - $2.04\left(\mathrm{~m}, 2 \mathrm{H}, \mathrm{CH}_{2}\right), 2.20-2.29\left(\mathrm{~m}, 2 \mathrm{H}, \mathrm{CH}_{2}\right), 4.24\left(\mathrm{~s}, 2 \mathrm{H}, \mathrm{N}-\mathrm{CH}_{2}-\mathrm{C}_{\mathrm{q}}\right)$, 4.89 - 5.01 (m, $\left.2 \mathrm{H}, \mathrm{CH}_{2, \text { olefin. }}\right), 5.15$ - $5.23(\mathrm{~m}, 1 \mathrm{H}, \mathrm{CH}), 5.77-5.87(\mathrm{~m}, 1 \mathrm{H}$, $\left.\mathrm{CH}_{\text {olefin. }}\right), 8.63-8.69 \mathrm{ppm}\left(\mathrm{m}, 8 \mathrm{H}, 8 \times \mathrm{CH}_{\text {perylene }}\right) .{ }^{13} \mathrm{C} \mathrm{NMR}\left(150 \mathrm{MHz}, \mathrm{CDCl}_{3}\right.$, $25^{\circ} \mathrm{C}$, TMS): $\delta=14.0,14.1,22.6,23.1,23.8,25.7,26.9,29.2,31.7,32.4,34.8,35.9$, 40.7, 45.6, 54.8, 114.2, 123.0, 123.1, 123.6, 126.6, 129.3, 129.6, 131.5, 134.6, 139.2, $164.3 \mathrm{ppm}$. UV/Vis $\left(\mathrm{CHCl}_{3}\right): \lambda_{\max }(E)=458.0(0.22), 488.8(0.61), 525.4 \mathrm{~nm}$ (1.00). Fluorescence $\left(\mathrm{CHCl}_{3}\right): \lambda_{\max }(I)=533.5(1.00), 575.8(0.52), 623.8 \mathrm{~nm}$ (0.12). Fluorescence quantum yield $\left(\lambda_{\text {exc }}=488 \mathrm{~nm}, E_{488 \mathrm{~nm} / 1 \mathrm{~cm}}=0.0294\right.$, reference: 2,9-bis-(1-hexylheptyl)anthra[2,1,9-def,6,5,10-d'e'f] diisoquinoline-1,3,8,10-tetra one (1) with $\Phi=1.00$ [40]): 1.00. MS: $\left(\mathrm{DEI}^{+} / 70 \mathrm{eV}\right): \mathrm{m} / z(\%): 780(51)\left[M^{+}\right], 711$ (8) $\left[M^{+}-\mathrm{C}_{5} \mathrm{H}_{9}\right], 586(28)\left[M^{+}-\mathrm{C}_{14} \mathrm{H}_{27}\right], 576(14)\left[M^{+}-\mathrm{C}_{15} \mathrm{H}_{29}\right], 404$ (100) $\left[M^{+}-\right.$ $\left.\mathrm{C}_{13} \mathrm{H}_{27}-\mathrm{C}_{14} \mathrm{H}_{27}\right], 390(23)\left[M^{+}-\mathrm{C}_{13} \mathrm{H}_{27}-\mathrm{C}_{15} \mathrm{H}_{29}\right]$. HMRS $\left(\mathrm{C}_{52} \mathrm{H}_{65} \mathrm{~N}_{2} \mathrm{O}_{4}\right)$ : Calcd. $\mathrm{m} / z .781 .494\left[M^{+}+\mathrm{H}\right]$; found $\mathrm{m} / z$. 781.496 .

2-(1-Octylnonyl)-9-(4-vinylphenyl)anthra[2,1,9-def,6,5,10-d'e'f']diisoqui noline-1,3,8,10-tetraone (6a): 9-(1-Ocytlnonyl)-2-benzopyrano[6', 5' 4':10,5,6] anthra[2,1,9-def]iso-quinoline-1,3,8,10-tetraone (494 $\mathrm{mg}, 0.784 \mathrm{mmol}$ ) 4-vinylaniline $(121 \mathrm{mg}, 1.02 \mathrm{mmol})$, imidazole $(6.0 \mathrm{~g})$ the quantity of a microspatulum of zincacetate dihydrate, acetic acid $(120 \mathrm{~mL}$, replacement of ethanol) and $2 \mathrm{~N}$ aqueous $\mathrm{HCl}(100 \mathrm{~mL})$ were allowed to react as was described for 2-(2,2-dibutylhept6-enyl)-9-(2-hydroxyethyl)anthra[2,1,9-def,6,5,10-d'e'f]diisoquinoline-1,3,8,10-t 
etraone (42) and purified by column separation ( $800 \mathrm{~mL}$ of silica gel, chloroform/ethanol 30:1, glas column grobes $780 \times 44 \mathrm{~mm})$. Yield $362 \mathrm{mg}(63 \%)$ light red solid, m.p. $>250^{\circ} \mathrm{C} . R_{\mathrm{f}}$-value (alumina, $\left.\mathrm{CHCl}_{3}\right)=0.49$. IR $(\mathrm{KBr}): \tilde{v}=$ $3091.5(\mathrm{w}), 2954.2(\mathrm{~m}), 2925.0(\mathrm{~m}), 2854.5(\mathrm{~m}), 1698.4(\mathrm{~s}), 1658.4(\mathrm{vs}), 1594.6$ (s), $1578.2(\mathrm{~m}), 1510.3(\mathrm{w}), 1483.5(\mathrm{w}), 1465.6(\mathrm{w}), 1433.8(\mathrm{w}), 1404.8(\mathrm{~m})$, $1343.9(\mathrm{~s}), 1301.8(\mathrm{w}), 1254.9(\mathrm{~m}), 1197.0(\mathrm{w}), 1176.7(\mathrm{w}), 1137.9(\mathrm{w}), 1124.8$ (w), $1112.7(\mathrm{w}), 988.2(\mathrm{w}), 970.0(\mathrm{w}), 908.6(\mathrm{w}), 846.4(\mathrm{w}), 810.5(\mathrm{~m}), 798.2(\mathrm{w})$, $746.6(\mathrm{~m}), 722.1(\mathrm{w}), 617.6(\mathrm{w}), 592.5(\mathrm{w}), 499.1 \mathrm{~cm}^{-1}$ (w). ${ }^{1} \mathrm{H}$ NMR $(600 \mathrm{MHz}$, $\left.\mathrm{CDCl}_{3}, 25^{\circ} \mathrm{C}, \mathrm{TMS}\right): \delta=0.83\left(\mathrm{t},{ }^{3}(\mathrm{H}, \mathrm{H})=7.0 \mathrm{~Hz}, 6 \mathrm{H}, 2 \times \mathrm{CH}_{3}\right), 1.20-1.38(\mathrm{~m}$, $\left.24 \mathrm{H}, 12 \times \mathrm{CH}_{2}\right), 1.85-1.91\left(\mathrm{~m}, 2 \mathrm{H}, \beta-\mathrm{CH}_{2}\right), 2.22-2.28\left(\mathrm{~m}, 2 \mathrm{H}, \beta-\mathrm{CH}_{2}\right), 5.16-$ $5.21\left(\mathrm{~m}, 1 \mathrm{H}, \alpha-\mathrm{CH}_{2}\right), 5.30\left(\mathrm{~m}, 1 \mathrm{H}, \mathrm{CH}_{2, \text { olefin. }}\right), 5.82-5.85\left(\mathrm{~m}, 1 \mathrm{H}, \mathrm{CH}_{2, \text { olefin. }}\right), 6.79$ - $6.84\left(\mathrm{~m}, 1 \mathrm{H}, \mathrm{CH}_{\text {olefin }}\right), 7.32-7.33\left(\mathrm{~m}, 2 \mathrm{H}, 2 \times \mathrm{CH}_{\text {aryl }}\right), 7.60-7.62(\mathrm{~m}, 2 \mathrm{H}, 2 \times$ $\left.\mathrm{CH}_{\text {aryl }}\right), 8.61-8.72 \mathrm{ppm}\left(\mathrm{m}, 8 \mathrm{H}, 8 \times \mathrm{CH}_{\text {perylene }}\right) .{ }^{13} \mathrm{C} \mathrm{NMR}\left(150 \mathrm{MHz}, \mathrm{CDCl}_{3}\right.$, $25^{\circ} \mathrm{C}$, TMS): $\delta=14.1,22.6,27.0,29.2,29.5,31.8,32.4,54.8,115.1,123.0,123.3$, $124.2,126.4,126.6,127.2,128.7,129.5,129.8,131.1,131.8,134.3,135.1,136.1$, $138.3,163.5,164.5 \mathrm{ppm}$. UV/Vis $\left(\mathrm{CHCl}_{3}\right): \lambda_{\max }(E)=459.4(0.22), 490.2(0.60)$, $526.8 \mathrm{~nm}(1.00)$. Fluorescence $\left(\mathrm{CHCl}_{3}\right): \lambda_{\max }(I)=535.3(1.00), 579.0(0.53), 626.3$ $\mathrm{nm}$ (0.13). Fluorescence quantum yield $\left(\lambda_{\mathrm{exc}}=489 \mathrm{~nm}, E_{489 \mathrm{~nm} / 1 \mathrm{~cm}}=0.0372\right.$; reference: 2,9-bis-(1-hexylheptyl)anthra[2,1,9-def,6,5,10-d'e'f]diiso- quinoline-1, 3,8,10-tetraone (1) with $\Phi=1.00$ [40]): 1.00. MS: $\left(\mathrm{DEI}^{+} / 70 \mathrm{eV}\right): \mathrm{m} / z(\%): 730$ (20) $\left[M^{+}\right], 617(2)\left[M^{+}-\mathrm{C}_{8} \mathrm{H}_{17}\right], 492(100)\left[M^{+}-\mathrm{C}_{17} \mathrm{H}_{35}\right]$. HMRS $\left(\mathrm{C}_{49} \mathrm{H}_{50} \mathrm{~N}_{2} \mathrm{O}_{4}\right)$ : Calcd. $m / z$. $731.384\left[M^{+}+\mathrm{H}\right]$; found $m / z$. 731.382 .

2-Allyl-9-(1-nonyldecyl)anthra[2,1,9-def,6,5,10-d'e'f] diisoquinoline-1,3,8, 10-tetraone: 9-(1-Nonyldecyl)-2-benzopyrano $\left[6^{\prime}, 5^{\prime} 4^{\prime}: 10,5,6\right]$ anthra[2,1,9-def] isoquinoline-1,3,8,10-tetraone (300 $\mathrm{mg}, 0.456 \mathrm{mmol})$, imidazole $(7.0 \mathrm{~g})$, allylamine (150 mg, $2.63 \mathrm{mmol}$ ), the quantity of a microspatulum of zincacetate dihydrate, acetic acid $(100 \mathrm{~mL}$, replacement of ethanol) and $2 \mathrm{~N}$ aqueous $\mathrm{HCl}(150 \mathrm{~mL}$, precipitation with ice-cooling) were allowed to react as was described for 2-(2,2-dibutylhept-6-enyl)-9-(2-hydroxy-ethyl)anthra[2,1,9-def;6,5,10-d'eff]diiso quinoline-1,3,8,10-tetraone and purified by column separation $(500 \mathrm{~mL}$ of alumina, chloroform/n-pentane 2:1, glass column $500 \times 44 \mathrm{~mm}$ and then $800 \mathrm{~mL}$ of silica gel, chloroform/ethanol 80:1, glass column $780 \times 44 \mathrm{~mm}$ ). Yield $112 \mathrm{mg}$ (35\%) dark red solid, m.p. $>250^{\circ} \mathrm{C} . R_{\mathrm{f}}$-value (silica gel, $\left.\mathrm{CHCl}_{3} / \mathrm{EtOH} 80: 1\right)=$ 0.19. IR $(\mathrm{KBr}): \tilde{v}=2924.1(\mathrm{~s}), 2853.6(\mathrm{~m}), 1698.5(\mathrm{~s}), 1658.7(\mathrm{~s}), 1595.5(\mathrm{~s})$, $1578.0(\mathrm{~m}), 1506.2(\mathrm{w}), 1436.2(\mathrm{w}), 1404.2(\mathrm{~m}), 1376.5(\mathrm{w}), 1346.0(\mathrm{~m}), 1252.4$ (m), $1175.0(\mathrm{w}), 1096.2(\mathrm{w}), 993.6(\mathrm{w}), 937.0(\mathrm{w}), 851.4(\mathrm{w}), 809.9(\mathrm{~m}), 795.4(\mathrm{w})$, $746.9 \mathrm{~cm}^{-1}(\mathrm{~m}) .{ }^{1} \mathrm{H}$ NMR $\left(600 \mathrm{MHz}, \mathrm{CDCl}_{3}, 25^{\circ} \mathrm{C}\right.$, TMS $): \delta=0.83\left(\mathrm{t},{ }^{3}\right)(\mathrm{H}, \mathrm{H})=$ $\left.7.0 \mathrm{~Hz}, 6 \mathrm{H}, 2 \times \mathrm{CH}_{3}\right), 1.20-1.27\left(\mathrm{~m}, 28 \mathrm{H}, 14 \times \mathrm{CH}_{2}\right), 1.87\left(\mathrm{~m}, 2 \mathrm{H}, \alpha-\mathrm{CH}_{2}\right)$, $2.25\left(\mathrm{~m}, 2 \mathrm{H}, \alpha-\mathrm{CH}_{2}\right), 4.84\left(\mathrm{~d},{ }^{3}(\mathrm{H}, \mathrm{H})=5.8 \mathrm{~Hz}, 2 \mathrm{H}, \mathrm{N}-\mathrm{CH}_{2}\right), 5.18(\mathrm{~m}, 1 \mathrm{H}$, $\mathrm{N}-\mathrm{CH}), 5.25\left(\mathrm{~d},{ }^{3} /(\mathrm{H}, \mathrm{H})=10.5 \mathrm{~Hz}, 1 \mathrm{H}, \mathrm{CH}_{2, \text { olefin. }}\right), 5.37\left(\mathrm{~d},{ }^{3} /(\mathrm{H}, \mathrm{H})=16.3,1 \mathrm{H}\right.$, $\left.\mathrm{CH}_{2, \text { olefin. }}\right), 6.02\left(\mathrm{~m}, 1 \mathrm{H}, \mathrm{CH}_{\text {olefin. }}\right), 8.59-8.68 \mathrm{ppm}\left(\mathrm{m}, 8 \mathrm{H}, 8 \times \mathrm{CH}_{\text {perylene }}\right)$. UV/Vis $\left(\mathrm{CHCl}_{3}\right): \lambda_{\max }(E)=459(0.23), 489(0.60), 526 \mathrm{~nm}(1.00)$. Fluorescence $\left(\mathrm{CHCl}_{3}\right)$ : $\lambda_{\max }(I)=533(1.00), 577 \mathrm{~nm}(0.37)$. Fluorescence quantum yield $\left(\mathrm{CHCl}_{3}, \lambda_{\mathrm{exc}}=\right.$ 
$487 \mathrm{~nm}, E_{487 \mathrm{~nm} / 1 \mathrm{~cm}}=0.0304$; reference: 2,9-bis-(1-hexylheptyl)anthra[2,1,9-def; 6,5,10-d'e'f] diisoquinoline-1,3,8,10-tetraone (1) with $\Phi=1.00$ [40]): 1.00. MS $\left(\mathrm{DEI}^{+} / 70 \mathrm{eV}\right): m / z(\%): 696(78)\left[M^{+}\right], 430(100)\left[M^{+}-\mathrm{C}_{19} \mathrm{H}_{38}\right], 415(46)[430-$ $\mathrm{CH}_{3}$ ]. HMRS $\left(\mathrm{C}_{46} \mathrm{H}_{52} \mathrm{~N}_{2} \mathrm{O}_{4}\right)$ : Calcd. $\mathrm{m} / z$ : 696.393; found $\mathrm{m} / z$ : 696.394 .

2-(1-Nonyldecyl)-9-(4-vinylphenyl)anthra[2,1,9-def,6,5,10-d'e'f']diisoqui noline-1,3,8,10-tetraone (6b): Variant I: 9-(1-Nonyldecyl)-2-benzopyrano[6', $\left.5^{\prime} 4^{\prime}: 10,5,6\right]$ anthra[2,1,9-def $]$ isoquinoline-1,3,8,10-tetraone $\quad(500 \quad \mathrm{mg}, \quad 0.760$ mmol), imidazole $(6.0 \mathrm{~g}), 4$-amino-styrene $(161 \mathrm{mg}, 1.35 \mathrm{mmol})$, the quantity of a microspatulum of zincacetate dihydrate, acetic acid $(80 \mathrm{~mL}$, replacement of ethanol) and $2 \mathrm{~N}$ aqueous $\mathrm{HCl}(100 \mathrm{~mL})$ were allowed to react as was described for 2-(2,2-dibutylhept-6-enyl)-9-(2-hydroxyethyl)anthra[2,1,9-def;6,5,10-d'e'f] diisoquinoline-1,3,8,10-tetraone and purified by column separation $(800 \mathrm{~mL}$ silica gel, chloroform, glass column $780 \times 44 \mathrm{~mm})$. Yield $281 \mathrm{mg}(49 \%)$ dark red solid. Variant II: 9-(4-Phenylacrylsäure)-2-benzopyrano $\left[6^{\prime}, 5^{\prime} 4^{\prime}:\right.$ : 10,5,6]anthra [2,1,9-def] isoquinoline-1,3,8,10-tetraone (400 mg, $0.744 \mathrm{mmol})$, 1-nonyldecylamine (274 $\mathrm{mg}, 0.967 \mathrm{mmol})$ imidazole $(6.0 \mathrm{~g})$, the quantity of a microspatulum of zincacetate dihydrate, acetic acid $(100 \mathrm{~mL}$, replacement of ethanol) and $2 \mathrm{~N}$ aqueous $\mathrm{HCl}(120 \mathrm{~mL})$ were allowed to react as was described for 2-(2,2-dibutylhept-6-enyl)-9-(2-hydroxyethyl)-anthra-[2,1,9-def,6,5,10-d'e'f] diis oquinoline-1,3,8,10-tetraone and purified by column separation $(500 \mathrm{~mL}$ of silica gel, chloroform, glass colmn $500 \times 44 \mathrm{~mm})$. Yield $<1 \%$, m.p. $>250^{\circ} \mathrm{C}$. $R_{\mathrm{f}}$-value (silica gel, $\left.\mathrm{CHCl}_{3}\right)=0.43 . \mathrm{IR}(\mathrm{KBr}): \tilde{v}=3092.1(\mathrm{~m}), 2954.3(\mathrm{~m})$, $2924.5(\mathrm{~s}), 2854.0(\mathrm{~m}), 1698.6(\mathrm{~s}), 1658.6(\mathrm{~s}), 1594.6(\mathrm{~s}), 1578.1(\mathrm{~m}), 1510.5(\mathrm{~m})$, $1483.3(\mathrm{w}), 1465.6(\mathrm{w}), 1433.9(\mathrm{~m}), 1404.6(\mathrm{~m}), 1344.2(\mathrm{~s}), 1302.0(\mathrm{w}), 1255.2(\mathrm{~s})$, $1197.0(\mathrm{w}), 1177.4(\mathrm{~m}), 1137.9(\mathrm{w}), 1125.1(\mathrm{w}), 1113.5(\mathrm{w}), 988.1(\mathrm{w}), 963.5(\mathrm{w})$, $921.3(\mathrm{w}), 847.5(\mathrm{w}), 810.5(\mathrm{~m}), 798.2(\mathrm{~m}), 757.9(\mathrm{w}), 746.6(\mathrm{~m}), 722.1 \mathrm{~cm}^{-1}(\mathrm{w})$. ${ }^{1} \mathrm{H}$ NMR (600 MHz, $\mathrm{CDCl}_{3}, 25^{\circ} \mathrm{C}$, TMS): $\delta=0.83\left(\mathrm{t},{ }^{3} \mathcal{J}(\mathrm{H}, \mathrm{H})=7.1 \mathrm{~Hz}, 6 \mathrm{H}, 2 \times\right.$ $\left.\mathrm{CH}_{3}\right), 1.21-1.32\left(\mathrm{~m}, 28 \mathrm{H}, 14 \times \mathrm{CH}_{2}\right), 1.84-1.90\left(\mathrm{~m}, 2 \mathrm{H}, \beta-\mathrm{CH}_{2}\right), 2.22-2.28$ $\left(\mathrm{m}, 2 \mathrm{H}, \beta-\mathrm{CH}_{2}\right), 5.19(\mathrm{~m}, 1 \mathrm{H}, \alpha-\mathrm{CH}), 5.35\left(\mathrm{~d},{ }^{3} \mathcal{J}(\mathrm{H}, \mathrm{H})=10.9 \mathrm{~Hz}, 1 \mathrm{H}, \mathrm{CH}_{2, \text { olefin }}\right)$, $5.84\left(\mathrm{~d},{ }^{3} \mathcal{H}(\mathrm{H}, \mathrm{H})=17.6 \mathrm{~Hz}, 1 \mathrm{H}, \mathrm{CH}_{2, \text { olefin }}\right), 6.81\left(\mathrm{dd},{ }^{3} \mathcal{H}(\mathrm{H}, \mathrm{H})=17.6,10.9 \mathrm{~Hz}, 1 \mathrm{H}\right.$, $\left.\mathrm{CH}_{\text {olefin }}\right), 7.32\left(\mathrm{~d},{ }^{3} \mathcal{J}(\mathrm{H}, \mathrm{H})=8.3 \mathrm{~Hz}, 2 \mathrm{H}, \mathrm{CH}_{\text {aryl }}\right), 7.61\left(\mathrm{~d},{ }^{3} \mathcal{J}(\mathrm{H}, \mathrm{H})=8.3 \mathrm{~Hz}, 2 \mathrm{H}\right.$, $\left.\mathrm{CH}_{\text {aryl }}\right), 8.62-8.73 \mathrm{ppm}\left(\mathrm{m}, 8 \mathrm{H}, 8 \times \mathrm{CH}_{\text {perylene }}\right) .{ }^{13} \mathrm{C} \mathrm{NMR}\left(151 \mathrm{MHz}, \mathrm{CDCl}_{3}\right.$, $\left.25^{\circ} \mathrm{C}, \mathrm{TMS}\right): \delta=14.4,32.0,27.3,29.6,29.8,30.0,32.2,32.7,55.1,115.5,123.4$, $123.6,126.7,127.0,127.5,129.0,129.9,130.2,132.2,134.6,134.7,135.5,136.4$, 138.6, $163.9 \mathrm{ppm}$. UV/Vis $\left(\mathrm{CHCl}_{3}\right): \lambda_{\max }(E)=459(0.22), 490(0.60), 527 \mathrm{~nm}$ (1.00). Fluorescence $\left(\mathrm{CHCl}_{3}\right): \lambda_{\max }(I)=534(1.00), 578 \mathrm{~nm}(0.40)$. Fluorescence quantum yield $\left(\mathrm{CHCl}_{3}, \lambda_{\text {exc }}=489 \mathrm{~nm}, E_{489 \mathrm{~nm} / 1 \mathrm{~cm}}=0.0319\right.$; reference: 2,9 -bis-(1hexylheptyl)anthra[2,1,9-def6,5,10-d'e'f]diisoquinoline-1,3,8,10-tetraone (1) with $\Phi=1.00)[40]: 1.00 . \mathrm{MS}\left(\mathrm{DEI}^{+} / 70 \mathrm{eV}\right): \mathrm{m} / z(\%): 758(27)\left[M^{+}\right], 492(100)\left[M^{+}-\right.$ $\left.\mathrm{C}_{19} \mathrm{H}_{38}\right], 373(16)\left[\mathrm{C}_{24} \mathrm{H}_{7} \mathrm{NO}_{4}\right]$. HMRS $\left(\mathrm{C}_{51} \mathrm{H}_{55} \mathrm{~N}_{2} \mathrm{O}_{4}\right)$ : Calcd. $m / z: 759.416\left[M^{+}+\right.$ $\mathrm{H}]$; found $\mathrm{m} / \mathrm{z}$ : 759.418 .

2-Allyl-9-(2,5-di-tert-butylphenyl)anthra[2,1,9-def,6,5,10-d'e'f] diisoquin oline-1,3,8,10-tetraone:9-(2,5-Di-tert-butylphenyl)-2-benzopyrano[6' $6^{\prime} 4^{\prime}: 10$, 
5,6]anthra[2,1,9-def]-isoquinoline-1,3,8,10-tetraone (120 mg, $0.207 \mathrm{mmol}$ ), imidazole $(3.0 \mathrm{~g})$, allylamine $(35 \mathrm{mg}(0.63 \mathrm{mmol})$, the quantity of a microspatulum of zincacetate dihydrate, ethanol $(40 \mathrm{~mL})$ and $2 \mathrm{~N}$ aqueous $\mathrm{HCl}$ $(120 \mathrm{~mL})$ were allowed to react as was described for 2-(2,2-dibutylhept-6enyl)-9-(2-hydroxyethyl)anthra[2,1,9-def,6,5,10-d'e'f] diisoquinoline-1,3,8,10-tet raone and purified by column separation $(500 \mathrm{~mL}$ of silica gel, chloroform/ethanol 30:1, glass column $500 \times 44 \mathrm{~mm})$. Yield: $71 \mathrm{mg}(55 \%)$ dark red solid, m.p. $>250^{\circ} \mathrm{C} . R_{\mathrm{f}}$-value (silica gel, $\left.\mathrm{CHCl}_{3} / \mathrm{EtOH} 30: 1\right)=0.21$. IR $(\mathrm{KBr}): \tilde{v}$ = $2961.5(\mathrm{~m}), 2924.2(\mathrm{~m}), 1701.0(\mathrm{~m}), 1663.1(\mathrm{~m}), 1594.3(\mathrm{~m}), 1578.7(\mathrm{~m}), 1507.5$ (w), $1435.8(\mathrm{w}), 1402.1(\mathrm{~m}), 1359.5(\mathrm{~m}), 1252.8(\mathrm{w}), 1177.8(\mathrm{w}), 1125.7(\mathrm{w}), 997.5$ (w), $854.3(w), 826.5(w), 811.6(w), 794.1(w), 750.2(w), 734.0 \mathrm{~cm}^{-1}(w) .{ }^{1} \mathrm{H}$ NMR (600 MHz, $\mathrm{CDCl}_{3}, 25^{\circ} \mathrm{C}$, TMS): $\delta=1.23$ (s, $9 \mathrm{H}$, tert-butyl), 1.27 (s, $9 \mathrm{H}$, tert-butyl), $4.79\left(\mathrm{~d},{ }^{3} \mathcal{J}(\mathrm{H}, \mathrm{H})=5.9 \mathrm{~Hz}, 2 \mathrm{H}, \mathrm{N}-\mathrm{CH}_{2}\right), 5.19\left(\mathrm{~d},{ }^{3} /(\mathrm{H}, \mathrm{H})=10.3 \mathrm{~Hz}, 1\right.$ $\left.\mathrm{H}, \mathrm{CH}_{2, \text { olefin. }}\right), 5.31\left(\mathrm{~d},{ }^{3} \mathcal{J}(\mathrm{H}, \mathrm{H})=17.0 \mathrm{~Hz}, 1 \mathrm{H}, \mathrm{CH}_{2, \text { olefin. }}\right), 5.97\left(\mathrm{~m}, 1 \mathrm{H}, \mathrm{CH}_{\text {olefin. }}\right)$, $6.97\left(\mathrm{~s}, 1 \mathrm{H}, \mathrm{CH}_{\text {aryl }}\right), 7.41\left(\mathrm{~d},{ }^{3} \mathcal{J}(\mathrm{H}, \mathrm{H})=8.6 \mathrm{~Hz}, 1 \mathrm{H}, \mathrm{CH}_{\text {aryl }}\right), 7.53\left(\mathrm{~d},{ }^{3} \mathcal{J}(\mathrm{H}, \mathrm{H})=8.6\right.$ $\left.\mathrm{Hz}, 1 \mathrm{H}, \mathrm{CH}_{\text {ary }}\right), 8.60-8.71 \mathrm{ppm}\left(\mathrm{m}, 8 \mathrm{H}, \mathrm{CH}_{\text {perylene }}\right)$. UV/Vis $\left(\mathrm{CHCl}_{3}\right): \lambda_{\max }(E)=$ 458 (0.22), $490(0.60), 526 \mathrm{~nm}(1.00)$. Fluorescence $\left(\mathrm{CHCl}_{3}\right): \lambda_{\max }(I)=534$ (1.00), $575(0.35), 622 \mathrm{~nm}$ (0.06). Fluorescence quantum yield $\left(\mathrm{CHCl}_{3}, \lambda_{\mathrm{exc}}=488 \mathrm{~nm}\right.$, $E_{488 \mathrm{~nm} / 1 \mathrm{~cm}}=0.0571$; reference: 2,9-bis-(1-hexylheptyl)anthra- [2,1,9-def;6,5,10d'e'f] diisoquinoline-1,3,8,10-tetraone (1) with $\Phi=1.00$ [40]): 1.00. $\mathrm{MS}_{\left(\mathrm{DEI}^{+} / 70\right.}$ eV): $m / z$ (\%): $618(3)\left[M^{+}\right], 561(100)\left[M^{+}-\mathrm{C}_{4} \mathrm{H}_{9}\right.$ (isobutene)], 545 (7) [561 $\left.\mathrm{CH}_{3}\right]$. HMRS $\left(\mathrm{C}_{41} \mathrm{H}_{34} \mathrm{~N}_{2} \mathrm{O}_{4}\right)$ : Calcd. $\mathrm{m} / z$. 618.252; found $\mathrm{m} / z$ : 618.253 .

2-Allyl-9-(2,2-dibutyl-hept-6-enyl)anthra[2,1,9-def,6,5,10-d'e'f] diisoquin oline-1,3,8,10-tetraone (14): 9-Allyl-2-benzopyrano[6',5'4':10,5,6]anthra [2,1, 9-def] isoquinoline-1,3,8,10-tetraone (1.00 g, $2.32 \mathrm{mmol})$, imidazole $(5.0 \mathrm{~g})$; 2,2-dibutylhept-6-enylamine ( $672 \mathrm{mg}, 2.78 \mathrm{mmol})$, the quantity of a microspatulum of zincacetate dihydrate, ethanol $(15 \mathrm{~mL})$ and $2 \mathrm{~N}$ aqueous $\mathrm{HCl}(80 \mathrm{~mL})$ were allowed to react as was described for 2-(2,2-dibutylhept-6-enyl)-9-(2-hydroxyethyl) anthra[2,1,9-def,6,5,10-d'eff] diisoquinoline-1,3,8,10-tetraone and purified by column separation ( $800 \mathrm{~mL}$ of silica gel, chloroform/ethanol 50:1, glass column $800 \times 42 \mathrm{~mm}$ ) and then $500 \mathrm{~mL}$ of alumina, chloroform/ethanol 50:1, glass column $780 \times 44 \mathrm{~mm}$ ). The material was dissolved in the minimal amount of chloroform and treated with vapour of methanol at room temperature for isothermal distillation.Yield $807 \mathrm{mg}$ (54\%) red needles, m.p. $>250^{\circ} \mathrm{C}$. $R_{\mathrm{f}}$-value (alumina; $\mathrm{CHCl}_{3}$ ) = 0.38. IR (ATR): $\tilde{v}=2928.8(\mathrm{~s}), 2858.9(\mathrm{~m}), 1692.8(\mathrm{~s}), 1652.1$ (vs), $1591.5(\mathrm{~s}), 1507.1$ (w), 1433,5 (m), $1403.7(\mathrm{~m}), 1368.7 \mathrm{w}, 1325.2(\mathrm{~s}), 1246.0(\mathrm{~m})$, $1179.7(\mathrm{w}), 1091.5(\mathrm{w}), 1011.2(\mathrm{w}), 925.4(\mathrm{w}), 849.2(\mathrm{w}), 808.6(\mathrm{~m}), 792.5(\mathrm{w})$, $748.6(\mathrm{~m}), 655.5 \mathrm{~cm}^{-1}(\mathrm{w}) .{ }^{1} \mathrm{H}$ NMR $\left(600 \mathrm{MHz}, \mathrm{CDCl}_{3}, 25^{\circ} \mathrm{C}, \mathrm{TMS}\right): \delta=0.89(\mathrm{t}$, $\left.{ }^{3} \mathcal{H}(\mathrm{H}, \mathrm{H})=7.1 \mathrm{~Hz}, 6 \mathrm{H}, 2 \times \mathrm{CH}_{3}\right), 1.25-1.35\left(\mathrm{~m}, 14 \mathrm{H}, 7 \times \mathrm{CH}_{2}\right), 1.42-1.47(\mathrm{~m}, 2$ $\left.\mathrm{H}, \mathrm{CH}_{2}\right), 2.00\left(\mathrm{~m}, 2 \mathrm{H}, \mathrm{CH}_{2}\right), 4.22\left(\mathrm{~s}, 2 \mathrm{H}, \mathrm{N}-\mathrm{CH}_{2}\right), 4.84\left(\mathrm{~d}, 2 \mathrm{H},{ }^{3}\right)(\mathrm{H}, \mathrm{H})=5.9$ $\left.\mathrm{Hz}, \mathrm{N}-\mathrm{CH}_{2}\right), 4.89$ - $4.90\left(\mathrm{~m}, 1 \mathrm{H}, \mathrm{CH}_{2, \text { olefin. }}\right), 4.91-4.92\left(\mathrm{~m}, 1 \mathrm{H}, \mathrm{CH}_{2, \text { olefin. }}\right), 4.96$ $4.97\left(\mathrm{~m}, 1 \mathrm{H}, \mathrm{CH}_{2, \text { olefin. }}\right), 4.99-5.00\left(\mathrm{~m}, 1 \mathrm{H}, \mathrm{CH}_{2, \text { olefin. }}\right), 5.24-5.25(\mathrm{~m}, 1 \mathrm{H}$, $\mathrm{CH}_{2, \text { olefin. }}$ ), $5.26-5.27\left(\mathrm{~m}, 1 \mathrm{H}, \mathrm{CH}_{2, \text { olefin. }}\right), 5.36-5.37\left(\mathrm{~m}, 1 \mathrm{H}, \mathrm{CH}_{2, \text { olefin. }}\right), 5.39$ - 
$5.40\left(\mathrm{~m}, 1 \mathrm{H}, \mathrm{CH}_{2, \text { olefin. }}\right), 5.78-5.85$ (m, $\left.1 \mathrm{H}, \mathrm{CH}_{\text {olefin. }}\right), 6.00-6.07$ (m, $1 \mathrm{H}, \mathrm{CH}_{\text {ole- }}$ fin.), $8.55-8.57$ (m, $\left.4 \mathrm{H}, \mathrm{CH}_{\text {aromat. }}\right), 8.61-8.83$ (m, $\left.2 \mathrm{H}, \mathrm{CH}_{\text {aromat. }}\right), 8.66-8.67 \mathrm{ppm}$ $\left(\mathrm{m}, 2 \mathrm{H}, \mathrm{CH}_{\text {aromat. }}\right){ }^{13} \mathrm{C} \mathrm{NMR}\left(150 \mathrm{MHz}, \mathrm{CDCl}_{3}, 25^{\circ} \mathrm{C}, \mathrm{TMS}\right): \delta=14.4,23.3,24.0$, 26.0, 35.1, 36.1, 36.2, 40.9, 42.8, 114.5, 118.2, 123.1, 123.3, 123.4, 123.9, 126.5, $126.7,129.4,130.0,131.6,131.7,132.2,134.5,135.0,139.4,163.3,164.4$ ppm. UV/Vis $\left(\mathrm{CHCl}_{3}\right): \lambda_{\max }(\varepsilon)=456.8(18100), 488.0$ (49700), $524.4 \mathrm{~nm}(82500)$. Fluorescence $\left(\mathrm{CHCl}_{3}\right): \lambda_{\max }(I)=533.0(1.00), 574.5(0.52), 623.5 \mathrm{~nm}(0.12)$. Fluorescence quantum yield $\left(\lambda_{\text {exc }}=488 \mathrm{~nm}, E_{488 \mathrm{~nm} / 1 \mathrm{~cm}}=0.0306\right.$, reference: 2,9-bis-(1-hexylheptyl)anthra[2,1,9-def,6,5,10-d'e'f]-diisoquinoline-1,3,8,10-tetr aone (1) with $\Phi=1.00$ [40]): 1.00. MS: (DEI $/ 70 \mathrm{eV}): \mathrm{m} / z(\%): 638$ (90) $\left[M^{+}\right]$, 444 (100) $\left[M^{+}-\mathrm{C}_{14} \mathrm{H}_{27}\right], 429(35)\left[M^{+}-\mathrm{C}_{14} \mathrm{H}_{27}-\mathrm{CH}_{3}\right], 415(8)\left[M^{+}-\mathrm{C}_{14} \mathrm{H}_{27}-2\right.$ $\left.\times \mathrm{CH}_{3}\right] . \mathrm{C}_{42} \mathrm{H}_{42} \mathrm{~N}_{2} \mathrm{O}_{4}$ (638.8): Calcd. C 78.97, $\mathrm{H}$ 6.96, $\mathrm{N} 4.39$; found $\mathrm{C} 78.89, \mathrm{H}$ 6.63, N 4.40 .

2-\{2-(2,2-Dibutylhept-6-enyl)-9-(2-ethoxyethyl)anthra[2,1,9-def,6,5,10-d ' $e$ ' $f$ ' diisoquinoline-1,3,8,10-tetraon\}-9-(2,2-dibutylhept-6-enyl)-anthra[2,

1,9-def,6,5,10-d'e'f] diisoquinoline-1,3,8,10-tetraone (12): 2-(2,2-Dibutyl-hept-6enyl)-9-(2-hydroxyethyl)-anthra[2,1,9-def,6,5,10-d'e'f] diisoquinoline-1,3,8,10-te traone $(700 \mathrm{mg}, 1.09 \mathrm{mmol})$ was dissolved in refluxing dichloromethane (450 $\mathrm{mL})$, treated with diisopropylethylamine $(7 \mathrm{~mL}$, dark red solution), treated dropwise with methanesulfonicchloride $(3.0 \mathrm{~mL}, 39 \mathrm{mmol})$, heated with reflux for $3 \mathrm{~h}$, allowed to cool, treated with distilled water $(250 \mathrm{~mL})$ shaken with $2 \mathrm{~N}$ aqueous $\mathrm{HCl}(150 \mathrm{~mL})$, evaporated, dissolved in the minimal amount of chloroform, precipitated with methanol, colled by vaccum filtration (D4 micro glass filter), dried in air and purified by column separation $(100 \mathrm{~mL}$ of silica gel, chloroform/ethanol 30:1, second fraction, glass column $800 \times 44 \mathrm{~mm}$, and a second column separation with silica gel, chloroform/ethanol 30:1). Yield 496 $\mathrm{mg}(72 \%)$ red, only sparingly soluble solid, m.p. $>250^{\circ} \mathrm{C} . R_{\mathrm{f}}$-value (silica gel, $\left.\mathrm{CHCl}_{3} / \mathrm{EtOH} 30: 1\right)=0.12$. IR (ATR): $\tilde{v}=3519.7$ (w,br.), 3070.6 (w), 2952.7 (m), $2928.8(\mathrm{~m}), 2859.8(\mathrm{~m}), 1692.9$ (s), 1645.4 (vs), 1592.8 (vs), 1576.3 (m), $1506.1(\mathrm{w}), 1482.1(\mathrm{w}), 1457.2(\mathrm{w}), 1437.6(\mathrm{~m}), 1402.9(\mathrm{~m}), 1355.7(\mathrm{~s}), 1248.4$ (m), $1199.2(\mathrm{w}), 1169.2(\mathrm{w}), 1125.9(\mathrm{w}), 1059.5(\mathrm{w}), 1024.5(\mathrm{w}), 907.6(\mathrm{w}), 855.8$ (w), $808.7(\mathrm{~m}), 793.6(\mathrm{w}), 744.0(\mathrm{~m}), 667.9(\mathrm{w}), 640.2 \mathrm{~cm}^{-1}(\mathrm{w}) .{ }^{1} \mathrm{H}$ NMR (600 $\mathrm{MHz}, \mathrm{CDCl}_{3}, 25^{\circ} \mathrm{C}$, TMS): $\delta=0.89\left(\mathrm{t},{ }^{3} \mathcal{J}(\mathrm{H}, \mathrm{H})=7.1 \mathrm{~Hz}, 12 \mathrm{H}, 4 \times \mathrm{CH}_{3}\right), 1.25-$ $1.35\left(\mathrm{~m}, 28 \mathrm{H}, 14 \mathrm{CH}_{2}\right), 1.42-1.48\left(\mathrm{~m}, 4 \mathrm{H}, 2 \times \mathrm{CH}_{2}\right), 2.00-2.03(\mathrm{~m}, 4 \mathrm{H}, 2 \times$ $\left.\mathrm{C}_{\mathrm{q}}-\mathrm{CH}_{2}\right), 4.04\left(\mathrm{t},{ }^{3} J(\mathrm{H}, \mathrm{H})=5.2 \mathrm{~Hz}, 4 \mathrm{H}, 2 \times \mathrm{N}-\mathrm{CH}_{2}-\mathrm{CH}_{2}\right), 4.24(\mathrm{~s}, 4 \mathrm{H}, 2 \times$ $\left.\mathrm{N}-\mathrm{CH}_{2}\right), 4.51\left(\mathrm{t},{ }^{3} \mathcal{J}(\mathrm{H}, \mathrm{H})=5.4 \mathrm{~Hz}, 4 \mathrm{H}, 2 \times \mathrm{CH}_{2}-\mathrm{OH}\right), 4.89-4.91(\mathrm{~m}, 2 \mathrm{H}, 2 \times$ $\mathrm{CH}_{2 \text {,olefin. }}$ ), $4.96-4.99\left(\mathrm{~m}, 2 \mathrm{H}, 2 \times \mathrm{CH}_{2 \text {,olefin. }}\right), 5.78-5.85\left(\mathrm{~m}, 2 \mathrm{H}, 2 \times \mathrm{CH}_{\text {olefin. }}\right)$, 8.58 - $8.70 \mathrm{ppm}\left(\mathrm{m}, 16 \mathrm{H}, 16 \times \mathrm{CH}_{\text {perylene }}\right) .{ }^{13} \mathrm{C} \mathrm{NMR}\left(150 \mathrm{MHz}, \mathrm{CDCl}_{3}, 25^{\circ} \mathrm{C}\right.$, TMS): $\delta=14.2,23.0,23.7,25.7,34.8,35.8,35.9,40.7,43.0,45.6,61.4,114.2$, $122.7,122.8,123.2,123.5,125.9,126.1,128.9,129.3,131.0,131.4,133.8,134.6$, 139.2, $164.0 \mathrm{ppm}$. UV/Vis $\left(\mathrm{CHCl}_{3}\right): \lambda_{\max }(E)=458.6(0.23), 489.6(0.61), 526.2$ $\mathrm{nm}(1.00)$. Fluorescence $\left(\mathrm{CHCl}_{3}\right): \lambda_{\max }(I)=537.8(1.00), 577.8(0.52), 625.5 \mathrm{~nm}$ (0.13). Fluorescence quantum yield $\left(\lambda_{\mathrm{exc}}=488 \mathrm{~nm}, E_{488 \mathrm{~nm} / 1 \mathrm{~cm}}=0.0274\right.$; reference: 
2,9-bis-(1-hexylheptyl)anthra[2,1,9-def,6,5,10-d'e'f] diisoquinoline-1,3,8,10-tetra one (1) with $\Phi=1.00$ [40]): 1.00. MS: $\left(\mathrm{DEI}^{+} / 70 \mathrm{eV}\right): \mathrm{m} / z(\%): 1267(<1)\left[M^{+}\right]$, $642(100)\left[M^{+}-\mathrm{C}_{41} \mathrm{H}_{41} \mathrm{~N}_{2} \mathrm{O}_{4}\right], 626(24)\left[M^{+}-\mathrm{C}_{41} \mathrm{H}_{41} \mathrm{~N}_{2} \mathrm{O}_{5}\right], 448(64)\left[M^{+}-\right.$ $\left.\mathrm{C}_{41} \mathrm{H}_{41} \mathrm{~N}_{2} \mathrm{O}_{4}-\mathrm{C}_{14} \mathrm{H}_{27}\right], 405(99)\left[M^{+}-\mathrm{C}_{41} \mathrm{H}_{41} \mathrm{~N}_{2} \mathrm{O}_{4}-\mathrm{C}_{15} \mathrm{H}_{29}\right]$. HMRS $\left(\mathrm{C}_{82} \mathrm{H}_{82} \mathrm{~N}_{4} \mathrm{O}_{9}\right)$ : Calcd. $\mathrm{m} / \mathrm{z}$ : 1266.608; found $\mathrm{m} / \mathrm{z}:$ 1266.609. Byproduct I: 2-(2,2-Dibutylhept6-enyl)-9-(2-methoxyethyl)anthra[2,1,9-def,6,5,10-d'e'f'] diisoquinoline1,3,8,10-tetraone): Yield $39 \mathrm{mg}(5 \%)$ red solid, m.p. $>250^{\circ} \mathrm{C}$. $R_{\mathrm{f}}$-value (silica gel, $\left.\mathrm{CHCl}_{3} / \mathrm{EtOH} 30: 1\right)=0.12$. IR (ATR): $\left.\tilde{v}=3069.3(\mathrm{w}), 2953.6(\mathrm{~m}), 2929.38 \mathrm{~m}\right)$, $2860.5(\mathrm{~m}), 1694.9(\mathrm{~s}), 1650.8(\mathrm{vs}), 1593.0(\mathrm{~s}), 1577.2(\mathrm{~m}), 1506.6(\mathrm{w}), 1482.1(\mathrm{w})$, $1437.4(\mathrm{~m}), 1403.3(\mathrm{~m}), 1335.7(\mathrm{~s}), 1247.3(\mathrm{~m}), 1198.6(\mathrm{w}), 1173.9(\mathrm{w}), 1158.3$ (w), $1112.7(w), 1064.1(w), 1015.7(w), 909.7(w), 852.7(w), 808.7(m), 794.0$ (w), $744.8(\mathrm{~m}), 668.1(\mathrm{w}), 640.5 \mathrm{~cm}^{-1}(\mathrm{w}) .{ }^{1} \mathrm{H}$ NMR $\left(600 \mathrm{MHz}, \mathrm{CDCl}_{3}, 25^{\circ} \mathrm{C}\right.$, TMS): $\delta=0.89\left(\mathrm{t},{ }^{3} \mathcal{J}(\mathrm{H}, \mathrm{H})=7.0 \mathrm{~Hz}, 6 \mathrm{H}, 2 \times \mathrm{CH}_{3}\right), 1.26-1.46(\mathrm{~m}, 14 \mathrm{H}, 7 \times$ $\left.\mathrm{CH}_{2}\right), 2.00-2.03\left(\mathrm{~m}, 2 \mathrm{H}, \mathrm{C}_{\mathrm{q}}-\mathrm{CH}_{2}\right), 3.42\left(\mathrm{~s}, 3 \mathrm{H}, \mathrm{OCH}_{3}\right), 3.78\left(\mathrm{t},{ }^{3}\right)(\mathrm{H}, \mathrm{H})=5.3$ $\left.\mathrm{Hz}, 2 \mathrm{H}, \mathrm{N}-\mathrm{CH}_{2}-\mathrm{CH}_{2}\right), 4.23\left(\mathrm{~s}, 2 \mathrm{H}, \mathrm{N}-\mathrm{CH}_{2}\right), 4.48\left(\mathrm{t},{ }^{3}(\mathrm{H}, \mathrm{H})=5.3 \mathrm{~Hz}, 2 \mathrm{H}\right.$, $\left.\mathrm{MeO}-\mathrm{CH}_{2}\right), 4.89$ - 4.91 (m, $1 \mathrm{H}, \mathrm{CH}_{2, \text { olefin. }}$ ), 4.96 - 5.00 (m, $1 \mathrm{H}, \mathrm{CH}_{2, \text { olefin. }}$ ), 5.78 $5.85 \mathrm{ppm}\left(\mathrm{m}, 1 \mathrm{H}, \mathrm{CH}_{\text {olefin. }}\right){ }^{13} \mathrm{C} \mathrm{NMR}\left(150 \mathrm{MHz}, \mathrm{CDCl}_{3}, 25^{\circ} \mathrm{C}, \mathrm{TMS}\right): \delta=14.4$, 24.0, 25.9, 35.1, 36.2, 39.7, 40.9, 45.8, 59.1, 69.8, 114.5, 123.1, 123.3, 131.5, 131.7, $134.5,134.9,139.4,163.7,164.4 \mathrm{ppm}$. UV/Vis $\left(\mathrm{CHCl}_{3}\right): \lambda_{\max }(E)=458.4(0.23)$, $489.4(0.61), 525.8 \mathrm{~nm}$ (1.00). Fluorescence $\left(\mathrm{CHCl}_{3}\right): \lambda_{\max }(I)=534.8$ (1.00), $578.0(0.54), 626.3 \mathrm{~nm}(0.13)$. Fluorescence quantum yield $\left(\lambda_{\text {exc }}=488 \mathrm{~nm}, E_{488}\right.$ $\mathrm{nm} / 1 \mathrm{~cm}=0.02590$; reference: 2,9-bis-(1-hexylheptyl)anthra[2,1, 9-def,6,5,10$d^{\prime} e^{\prime} f$ ] diisoquinoline-1,3,8,10-tetraone (1) with $\left.\Phi=1.00\right)$ : 1.00. MS: $\left(\mathrm{DEI}^{+} / 70\right.$ $\mathrm{eV}): m / z(\%): 656(66)\left[M^{+}\right], 587(21)\left[M^{+}-\mathrm{C}_{5} \mathrm{H}_{9}\right], 462(39)\left[M^{+}-\mathrm{C}_{14} \mathrm{H}_{27}\right], 449$ (38) $\left[M^{+}-\mathrm{C}_{15} \mathrm{H}_{29}\right], 404(100)\left[M^{+}-\mathrm{C}_{14} \mathrm{H}_{27}-\mathrm{C}_{3} \mathrm{H}_{7} \mathrm{O}\right], 390(26)\left[M^{+}-\mathrm{C}_{15} \mathrm{H}_{29}-\right.$ $\left.\mathrm{C}_{3} \mathrm{H}_{7} \mathrm{O}\right]$. HMRS $\left(\mathrm{C}_{42} \mathrm{H}_{44} \mathrm{~N}_{2} \mathrm{O}_{5}\right)$ : Calcd. $\mathrm{m} / \mathrm{z}$. 656.325; found $\mathrm{m} / \mathrm{z}$. 656.326. Byproduct II: 2-(2,2-Dibutylhept-6-enyl)-9-(2-chlorethyl)anthra- [2,1,9-def, 6,5,10-d'e'f] diisoquinoline-1,3,8,10-tetraone (50): Yield $51 \mathrm{mg}(8 \%)$ red powder, m.p. $>250^{\circ} \mathrm{C} . R_{\mathrm{f}}$-value (silica gel; $\mathrm{CHCl}_{3} / \mathrm{EtOH} \mathrm{30:1)}=0.12$. IR (ATR): $\tilde{v}=3068.9(\mathrm{w}), 2952.2(\mathrm{~m}), 2932.6(\mathrm{~m}), 2868.7(\mathrm{~m}), 1695.4(\mathrm{~s}), 1658.9(\mathrm{vs})$, $1612.7(\mathrm{w}), 1592.7(\mathrm{~s}), 1578.3(\mathrm{~m}), 1507.4(\mathrm{w}), 1482.0(\mathrm{w}), 1457.9(\mathrm{w}), 1435.8$ (m), $1404.1(\mathrm{~m}), 1378.3(\mathrm{~m}), 1355.8(\mathrm{~m}), 1329.2(\mathrm{~s}), 1251.9(\mathrm{~m}), 1180.0(\mathrm{w})$, $1161.3(\mathrm{w}), 1124.0(\mathrm{w}), 1097.4(\mathrm{w}), 1015.1(\mathrm{~m}), 964.1(\mathrm{w}), 906.4(\mathrm{w}), 855.5(\mathrm{~m})$, $807.3(\mathrm{~m}), 794.0(\mathrm{~m}), 744.4(\mathrm{~m}), 668.3(\mathrm{w}), 643.3 \mathrm{~cm}^{-1}(\mathrm{w}) .{ }^{1} \mathrm{H}$ NMR $(600 \mathrm{MHz}$, $\left.\left.\mathrm{CDCl}_{3}, 25^{\circ} \mathrm{C}, \mathrm{TMS}\right): \delta=0.89\left(\mathrm{t},{ }^{3}\right)(\mathrm{H}, \mathrm{H})=7.0 \mathrm{~Hz}, 6 \mathrm{H}, 2 \times \mathrm{CH}_{3}\right), 1.25-1.35(\mathrm{~m}$, $\left.14 \mathrm{H}, 7 \times \mathrm{CH}_{2}\right), 1.42-1.47\left(\mathrm{~m}, 2 \mathrm{H}, \mathrm{CH}_{2}\right), 2.00-2.03\left(\mathrm{~m}, 2 \mathrm{H}, \mathrm{C}_{\mathrm{q}}-\mathrm{CH}_{2}\right), 3.90(\mathrm{t}$, $\left.{ }^{3}(\mathrm{H}, \mathrm{H})=6.8 \mathrm{~Hz}, 2 \mathrm{H}, \mathrm{N}-\mathrm{CH}_{2}-\mathrm{CH}_{2}\right), 4.23\left(\mathrm{~s}, 2 \mathrm{H}, \mathrm{N}-\mathrm{CH}_{2}\right), 4.61\left(\mathrm{t},{ }^{3}\right)(\mathrm{H}, \mathrm{H})=6.8$ $\left.\mathrm{Hz}, 2 \mathrm{H}, \mathrm{CH}_{2}-\mathrm{Cl}\right), 4.89-4.92\left(\mathrm{~m}, 1 \mathrm{H}, \mathrm{CH}_{2, \text { olefin. }}\right), 4.96-5.00\left(\mathrm{~m}, 1 \mathrm{H}, \mathrm{CH}_{\text {olefin. }}\right.$ ), 5.78 - 5.85 (m, $\left.1 \mathrm{H}, \mathrm{CH}_{\text {olefin. }}\right), 8.55$ - $8.68 \mathrm{ppm}\left(\mathrm{m}, 8 \mathrm{H}, 8 \times \mathrm{CH}_{\text {perylene }}\right) .{ }^{13} \mathrm{C} \mathrm{NMR}$ $\left(150 \mathrm{MHz}, \mathrm{CDCl}_{3}, 25^{\circ} \mathrm{C}, \mathrm{TMS}\right): \delta=14.4,23.3,24.0,26.0,35.1,36.1,40.7,40.9$, $41.6,45.9,114.5,123.0,123.1,123.5,124.0,129.4,131.5,131.9,134.3,135.2$, $139.4,163.5,164.4 \mathrm{ppm}$. UV/Vis $\left(\mathrm{CHCl}_{3}\right): \lambda_{\max }(E)=458.8(0.22), 489.6(0.60)$, $526.0 \mathrm{~nm}(1.00)$. Fluorescence $\left(\mathrm{CHCl}_{3}\right): \lambda_{\max }(I)=535.0(1.00), 577.5(0.53)$, 
$624.5 \mathrm{~nm}$ (0.12). Fluorescence quantum yield $\left(\lambda_{\mathrm{exc}}=488 \mathrm{~nm}, E_{\mathrm{nm} / 1 \mathrm{~cm}}=0.0393\right.$; reference: 2,9-bis-(1-hexylheptyl)anthra[2,1,9-def;6,5,10-d'e'f] diisoquinoline1,3,8,10-tetraone (1) with $\Phi=1.00$ [40]): 1.00. MS: $\left(\mathrm{DEI}^{+} / 70 \mathrm{eV}\right): \mathrm{m} / z(\%): 660$ (62) $\left[M^{+}\right], 626(71)\left[M^{+}-\mathrm{Cl}\right], 466(95)\left[M^{+}-\mathrm{C}_{14} \mathrm{H}_{27}\right], 432(100)\left[M^{+}-\mathrm{C}_{14} \mathrm{H}_{27}-\right.$ $\mathrm{Cl}], 404$ (54) $\left[M^{+}-\mathrm{C}_{14} \mathrm{H}_{27}-\mathrm{C}_{2} \mathrm{H}_{4} \mathrm{Cl}\right], 390$ (15) $\left[M^{+}-\mathrm{C}_{15} \mathrm{H}_{29}-\mathrm{C}_{2} \mathrm{H}_{4} \mathrm{Cl}\right]$. HMRS $\left(\mathrm{C}_{41} \mathrm{H}_{41} \mathrm{ClN}_{2} \mathrm{O}_{4}\right)$ : Calcd. $\mathrm{m} / \mathrm{z}$. 660.276; found $\mathrm{m} / \mathrm{z}$. 660.277.

2-(1-Hexylheptyl)-9-\{4-(2-(1-hexylheptyl)anthra[2,1,9-def;6,5,10-d'e' $\left.f^{\prime}\right]$ diisoquino-

line-1,3,8,10-tetraon-9-yl)but-2-enyl\}anthra[2,1,9-def,6,5,10-d'e'f'] diisoquin oline-1,3,8,10-tetraone(5):2-Allyl-9-(1-hexylheptyl)anthra[2,1,9-def,6,5,10-d'e'f ]diisoquinoline-1,3,8,10-tetraone $(300 \mathrm{mg}, 490 \mu \mathrm{mol})$ under argon atmosphere was disperged in dichloromethane $(10 \mathrm{~mL})$, heated to $50^{\circ} \mathrm{C}$, treated with second -generation Hoveyda-Grubbs-catalyst (3, $42 \mathrm{mg}, 50 \mu \mathrm{mol}$ darkening of the reaction mixture) heated to reflux for $2 \mathrm{~h}$ (red precipitate), evaporated in vacuo, treated with a small amount of chloroform and purified by column separation (800 mL of silica gel, chloroform/ethanol 40:1, glass column $780 \times 44 \mathrm{~mm}$, second red fraction after the starting material). Yield $112 \mathrm{mg}$ (19\%) reddish brown, sparingly soluble solid (cis/trans-mixture), m.p. $>250^{\circ} \mathrm{C} . R_{\mathrm{f}}$-value (silica gel, $\left.\mathrm{CHCl}_{3} / \mathrm{EtOH} 40: 1\right)=0.22$ (cis-isomer), 0.24 (trans-isomer). IR (KB\$r): $\tilde{v}=$ $2925.1(\mathrm{~m}), 2854.8(\mathrm{~m}), 1697.4(\mathrm{~m}), 1655.7$ (m), $1594.6(\mathrm{~m}), 1436.0(\mathrm{w}), 1404.0$ (m), $1339.9(\mathrm{~m}), 1249.5(\mathrm{w}), 1170.4(\mathrm{w}), 851.9(\mathrm{w}), 810.3(\mathrm{w}), 747.0 \mathrm{~cm}^{-1}(\mathrm{w}) .{ }^{1} \mathrm{H}$ NMR (600 MHz, $\left.\mathrm{CDCl}_{3}, 25^{\circ} \mathrm{C}, \mathrm{TMS}\right)$ : cis-isomer: $\delta=0.83\left(\mathrm{t},{ }^{3} \mathcal{J}(\mathrm{H}, \mathrm{H})=7.0 \mathrm{~Hz}\right.$, $\left.12 \mathrm{H}, 4 \times \mathrm{CH}_{3}\right), 1.16-1.41\left(\mathrm{~m}, 32 \mathrm{H}, 16 \times \mathrm{CH}_{2}\right), 1.83-1.89\left(\mathrm{~m}, 4 \mathrm{H}, 2 \times \beta-\mathrm{CH}_{2}\right)$, $2.21-2.28\left(\mathrm{~m}, 4 \mathrm{H}, 2 \times \beta-\mathrm{CH}_{2}\right), 5.15-5.21(\mathrm{~m}, 2 \mathrm{H}, 2 \times \alpha-\mathrm{CH}), 5.24-5.25(\mathrm{~m}, 4$ $\left.\mathrm{H}, 2 \times \mathrm{N}-\mathrm{CH}_{2}\right), 5.86-5.88\left(\mathrm{~m}, 2 \mathrm{H}, 2 \times \mathrm{CH}_{\text {olefin. }}\right), 8.57-8.74 \mathrm{ppm}(\mathrm{m}, 16 \mathrm{H}, 16 \times$ $\left.\mathrm{CH}_{\text {perylene }}\right)$; trans-isomer: $\delta=0.83\left(\mathrm{t},{ }^{3} J(\mathrm{H}, \mathrm{H})=7.9 \mathrm{~Hz}, 12 \mathrm{H}, 4 \times \mathrm{CH}_{3}\right), 1.16-1.41$ $\left(\mathrm{m}, 32 \mathrm{H}, 16 \times \mathrm{CH}_{2}\right), 1.83-1.89\left(\mathrm{~m}, 4 \mathrm{H}, 2 \times \beta-\mathrm{CH}_{2}\right), 2.21-2.28(\mathrm{~m}, 4 \mathrm{H}, 2 \times$ $\left.\beta-\mathrm{CH}_{2}\right), 4.83-4.84\left(\mathrm{~m}, 4 \mathrm{H}, 2 \times \mathrm{N}-\mathrm{CH}_{2}\right), 5.15-5.21(\mathrm{~m}, 2 \mathrm{H}, 2 \times \alpha-\mathrm{CH}), 6.09-$ $6.11\left(\mathrm{~m}, 2 \mathrm{H}, \mathrm{CH}_{\text {olefin. }}\right), 8.57-8.74 \mathrm{ppm}\left(\mathrm{m}, 16 \mathrm{H}, 16 \times \mathrm{CH}_{\text {perylene }}\right) . \mathrm{UV} / \mathrm{Vis}$ $\left(\mathrm{CHCl}_{3}\right): \lambda_{\max }(E)=459.8(0.23), 490.2(0.63), 527.2 \mathrm{~nm}$ (1.00). Fluorescence $\left(\mathrm{CHCl}_{3}\right): \lambda_{\max }(I)=534.0(1.00), 576.0 \mathrm{~nm}(0.35)$. Fluorescence quantum yield $\left(\lambda_{\text {exc }}=488 \mathrm{~nm}, E_{488 \mathrm{~nm} / \mathrm{lcm}}=0.0448\right.$; reference: 2,9-bis-(1-hexylheptyl)anthra[2,1, 9-def,6,5,10-d'e'f] diiso-quinoline-1,3,8,10-tetraone (1) with $\Phi=1.00$ [40]): 1.00 . MS (DEI+/70 eV): $m / z(\%): 1197(51)\left[M^{+}\right], 1014(58)\left[M^{+}-\mathrm{C}_{13} \mathrm{H}_{27}\right], 833(100)$ $\left[M^{+}-2 \times \mathrm{C}_{13} \mathrm{H}_{26}\right], 815$ (33), $624(22)\left[M^{+}-\mathrm{C}_{37} \mathrm{H}_{35} \mathrm{~N}_{2} \mathrm{O}_{4}\right], 611$ (12) $\left[M^{+}-\right.$ $\left.\mathrm{C}_{38} \mathrm{H}_{37} \mathrm{~N}_{2} \mathrm{O}_{4}\right], 572(11)\left[M^{+}-\mathrm{C}_{41} \mathrm{H}_{41} \mathrm{~N}_{2} \mathrm{O}_{4}\right], 442(92)\left[M^{+}-\mathrm{C}_{37} \mathrm{H}_{35} \mathrm{~N}_{2} \mathrm{O}_{4}-\mathrm{C}_{13} \mathrm{H}_{27}\right]$, 429 (15) $\left[M^{+}-\mathrm{C}_{38} \mathrm{H}_{37} \mathrm{~N}_{2} \mathrm{O}_{4}-\mathrm{C}_{13} \mathrm{H}_{26}\right], 417$ (56) $\left[M^{+}-\mathrm{C}_{39} \mathrm{H}_{37} \mathrm{~N}_{2} \mathrm{O}_{4}-\mathrm{C}_{13} \mathrm{H}_{26}\right], 416$ (21) $\left[M^{+}-\mathrm{C}_{39} \mathrm{H}_{38} \mathrm{~N}_{2} \mathrm{O}_{4}-\mathrm{C}_{13} \mathrm{H}_{26}\right], 403$ (8) $\left[M^{+}-\mathrm{C}_{40} \mathrm{H}_{39} \mathrm{~N}_{2} \mathrm{O}_{4}-\mathrm{C}_{13} \mathrm{H}_{26}\right], 390$ (92) $\left[M^{+}-\mathrm{C}_{41} \mathrm{H}_{40} \mathrm{~N}_{2} \mathrm{O}_{4}-\mathrm{C}_{13} \mathrm{H}_{26}\right], 373(30)\left[M^{+}-\mathrm{C}_{41} \mathrm{H}_{41} \mathrm{~N}_{3} \mathrm{O}_{4}-\mathrm{C}_{13} \mathrm{H}_{27}\right], 347$ (36) [ $M^{+}$ $-\mathrm{C}_{41} \mathrm{H}_{41} \mathrm{~N}_{3} \mathrm{O}_{4}-\mathrm{C}_{13} \mathrm{H}_{26}-\mathrm{CO}$ ], 345 (13), 69 (15), 55 (17). HMRS $\left(\mathrm{C}_{78} \mathrm{H}_{76} \mathrm{~N}_{4} \mathrm{O}_{8}\right)$ : Calcd. $\mathrm{m} / z$ : 1196.566; found $\mathrm{m} / z$. 1196.561 .

2-(2,5-Di-tert-butylphenyl)-9-\{4-(2-(2,5-di-tert-butylphenyl)anthra[2,1,9 -def,6,5,10-d' $e^{\prime} f$ ]diisoquinoline-1,3,8,10-tetraon-9-yl)but-2-enyl $\}$ anthra[2, 
1,9-def,6,5,10-d'e'f' diisoquinoline-1,3,8,10-tetraone:2-Allyl-9-(2,5-di-tert-bu tylphenyl)anthra[2,1,9-def;6,5,10-d'e'f] diisoquinoline-1,3,8,10-tetraone(50.0 mg, $80.8 \mu \mathrm{mol})$ under argon atmosphere was dissolved in dichloromethane $(5 \mathrm{~mL})$, treated with second-generation Hoveyda-Grubbs-catalyst (3, $9.15 \mathrm{mg}, 14.6$ $\mu \mathrm{mol}$ ), heated to reflux for $20 \mathrm{~h}$, terated with further second-generation Hoveyda-Grubbs-catalyst ( $5 \mathrm{mg}, 8 \mu \mathrm{mol}=10 \mathrm{Mol} \%$ ), heated to reflux for further 7 $\mathrm{h}$, stirred at room temperature for $36 \mathrm{~h}$, quenched by the addition of chloroform $(10 \mathrm{~mL})$ and distilled water $(10 \mathrm{~mL})$ stirred for $1 \mathrm{~h}$ shaken two timed with a misture of chloroform/water 1:1 with the collection of the organic phaseand purified by medium pressure chromatography (silica gel, chloroform/ethanol 40:1 at $10 \mathrm{~mL} \cdot \mathrm{min}^{-1}$, column $36 \times 460 \mathrm{~mm}$, second red fraction, and a second chromatography with silica gel, chloroform/ethanol 30:1). Yield $5.1 \mathrm{mg}$ (5\%) dark red, pigment-like powder. $R_{\mathrm{f}}$-value (silica gel, $\left.\mathrm{CHCl}_{3} / \mathrm{EtOH} 40: 1\right)=0.17$. UV/Vis $\left(\mathrm{CHCl}_{3}\right): \lambda_{\max }(E)=459.8(0.22), 491.0(0.58), 529.0 \mathrm{~nm}$ (1.00). Fluorescence $\left(\mathrm{CHCl}_{3}\right): \lambda_{\max }(I)=540.5(1.00), 578.0(0.74), 626.5 \mathrm{~nm}(0.17)$. Fluorescence quantum yield $\left(\lambda_{\mathrm{exc}}=489 \mathrm{~nm}, E_{488 \mathrm{~nm} / \mathrm{cm}}=0.0233\right.$; reference: 2,9-bis-(1hexylheptyl)anthra[2,1,9-def,6,5,10-d'e'f]diisoquinoline-1,3,8,10-tetraone (1) with $\Phi=1.00$ [40]): 1.00. MS (DEI $/ 70 \mathrm{eV}): \mathrm{m} / z$ (\%): 1151 (24) $\left[M^{+}-\mathrm{C}_{4} \mathrm{H}_{8}\right], 573$ (28), $521(100)\left[M^{+}-\mathrm{C}_{46} \mathrm{H}_{43} \mathrm{~N}_{2} \mathrm{O}_{4}\right], 505(15)\left[M^{+}-\mathrm{C}_{47} \mathrm{H}_{47} \mathrm{~N}_{2} \mathrm{O}_{4}\right]$. HMRS $\left(\mathrm{C}_{80} \mathrm{H}_{65} \mathrm{~N}_{4} \mathrm{O}_{8}\right)$ : Calcd. $m / z: 1209.480\left[M^{+}+\mathrm{H}\right]$; found $m / z: 1209.479$.

2-(1-Octylnonyl)-9-\{4'-(2-(1-octylnonyl)anthra[2,1,9-def,6,5,10-d' $\left.e^{\prime} f^{\prime}\right]$ diisoquino-

line-1,3,8,10-tetraon-9-yl)-stilben-4-yl\}anthra[2,1,9-def,6,5,10-d' $\left.e^{\prime} f^{\prime}\right]$ diis oquino-

line-1,3,8,10-tetraone(7a):2-(1-Octylnonyl)-9-(4-vinylphenyl)anthra[2,1,9-def, 6,5,10-d'e'f] diiso-quinoline-1,3,8,10-tetraone (6a, $203 \mathrm{mg}, 278 \mu \mathrm{mol})$ under argon atmosphere was dissolved in refluxing chloroform $(20 \mathrm{~mL})$, treated with second-generation Hoveyda-Grubbs-catalyst $(3,11.7 \mathrm{mg}, 13.8 \mu \mathrm{mol})$ heated with stirring (bath $80^{\circ} \mathrm{C}$ ) for $1 \mathrm{~h}$ and further $12 \mathrm{~h}$ at room temperature (no detectable reaction), treated with futher second-generation Hoveyda-Grubbs-catalyst (3, $13.0 \mathrm{mg}, 15.3 \mu \mathrm{mol}$ ) heated to reflux for $8 \mathrm{~h}$ (formation of a pigment-like red precipitate), collected by vacuum filtration (D4 micro glass filter) and thoroughly washed with hot chloroform to remove staring materials and by-products. Yield $66 \mathrm{mg}(17 \%)$ red pigment, m.p. $>250^{\circ} \mathrm{C}$. IR $(\mathrm{KBr}): \tilde{v}=$ $2952.7(\mathrm{~m}), 2924.0$ (s), $2853.6(\mathrm{~m}), 1698.2(\mathrm{~s}), 1660.1(\mathrm{~s}), 1594.4(\mathrm{~s}), 1578.3(\mathrm{~m})$, $1513.8(\mathrm{~m}), 1483.7(\mathrm{w}), 1464.7(\mathrm{w}), 1433.5(\mathrm{~m}), 1405.6(\mathrm{~m}), 1342.8(\mathrm{~s}), 1303.9$ (w), $1254.0(\mathrm{~m}), 1195.6(\mathrm{~m}), 1174.8(\mathrm{~m}), 1136.8(\mathrm{w}), 1124.2(\mathrm{w}), 1111.4(\mathrm{w})$, $1019.7(\mathrm{w}), 963.5(\mathrm{w}), 853.0(\mathrm{w}), 835.9(\mathrm{w}), 811.7(\mathrm{~m}), 798.8(\mathrm{~m}), 747.3(\mathrm{~m})$, $616.1(\mathrm{w}), 549.9(\mathrm{w}), 491.5(\mathrm{w}), 430.5 \mathrm{~cm}^{-1}(\mathrm{w})$. UV/Vis $\left(\mathrm{H}_{2} \mathrm{SO}_{4}\right): \lambda_{\max }(E)=404.8$ (0.10), $557.8(0.58), 602.4 \mathrm{~nm}(1.00)$. Fluorescence $\left(\mathrm{H}_{2} \mathrm{SO}_{4}\right): \lambda_{\max }(I)=623.0 \mathrm{~nm}$ (1.00). MS $\left(\mathrm{FAB}^{+} / 70 \mathrm{eV}\right): \mathrm{m} / z(\%): 1433\left[M^{+}\right], 879,823,801,765,749,731,613$, $460,391,307,154$.

2-(1-Nonyldecyl)-9-\{4'-(2-(1-nonyldecyl)anthra[2,1,9-def,6,5,10-d' e' $f^{\prime}$ 
]diiso-

quinline-1,3,8,10-tetraon-9-yl)-stilben-4-yl $\}$ anthra[2,1,9-def,6,5,10-d' $e^{\prime} f$ ]diisoquino-

line-1,3,8,10-tetraone(7b):2-(1-Nonyldecyl)-9-(4-vinylphenyl)anthra[2,1,9- $d$ ef,6,5,10-d'eff] diiso-quinoline-1,3,8,10-tetraone $(6 \mathrm{~b}, 150 \mathrm{mg}, 198 \mu \mathrm{mol})$ under argon atmosphere was dissolved in chloroform $(10 \mathrm{~mL})$, treated with second-generation Hoveyda-Grubbs-catalyst $(3,37.4 \mathrm{mg}, 44.0 \mu \mathrm{mol})$ heated with reflux (bath $80^{\circ} \mathrm{C}$ ) for $7 \mathrm{~h}$ (beginning of the formation of a precipiate of very fine, light red neddles after $2 \mathrm{~h}$ ), stirred at room temperature for $14 \mathrm{~h}$ and with reflux for $5 \mathrm{~h}$, collected by vacuum filtration (D4 micro glass filter) and washed with hot chloroform for removing staring matrials and by-products. Yield $12 \mathrm{mg}$ (4\%) dark red pigment, m.p. $>250^{\circ} \mathrm{C}$. IR $(\mathrm{KBr}): \tilde{v}=2923.5(\mathrm{~m}), 2852.8(\mathrm{~m})$, $1698.0(\mathrm{~s}), 1660.1(\mathrm{~s}), 1594.4(\mathrm{~s}), 1578.1(\mathrm{~m}), 1513.8(\mathrm{w}), 1465.0(\mathrm{w}), 1433.9(\mathrm{w})$, $1405.4(\mathrm{~m}), 1343.4(\mathrm{~s}), 1254.3(\mathrm{~m}), 1175.2(\mathrm{~m}), 1124.4(\mathrm{w}), 963.7(\mathrm{w}), 852.9(\mathrm{w})$, $811.7(\mathrm{~m}), 798.9(\mathrm{w}), 747.2(\mathrm{~m}), 549.9 \mathrm{~cm}^{-1}(\mathrm{w})$. UV/Vis $\left(\mathrm{H}_{2} \mathrm{SO}_{4}\right): \lambda_{\max }(E)=$ 317.6 (0.30), $402.8(0.12)$, $556.8(0.58), 601.8 \mathrm{~nm}(1.00)$. Fluorescence $\left(\mathrm{H}_{2} \mathrm{SO}_{4}\right)$ : $\lambda_{\max }(I)=631.5 \mathrm{~nm}(1.00) . \mathrm{MS}\left(\mathrm{FAB}^{+} / 70 \mathrm{eV}\right): \mathrm{m} / \mathrm{z}(\%): 1490\left[M^{+}\right], 1378,1225$, 1071, 919, $835\left[M^{+}-\mathrm{C}_{43} \mathrm{H}_{46} \mathrm{~N}_{2} \mathrm{O}_{4}\right], 829,793,759\left[M^{+}-\mathrm{C}_{49} \mathrm{H}_{49} \mathrm{~N}_{2} \mathrm{O}_{4}\right], 749,733$, 613, 596, 460, 307, 154.

2-(1-Hexylheptyl)-9-\{1-(2,2,13-tributyl-13-((1-hexylheptyl)anthra[2,1,9$\operatorname{de}\left\{6,5,10-d^{\prime} e^{\prime} f^{\prime}\right]$ diisoquinoline-1,3,8,10-tetraon)methyl)heptadec-7-enyl $\}$ anthra[2,1,9-def,6,5,10-d'e'f] diisoquinoline-1,3,8,10-tetraone(9):2-(2,2-Dibu tylhex-5-enyl)-9-(1-hexylheptyl)-anthra[2,1,9-def,6,5,10-d'e'f] diisoquinoline-1,3 ,8,10-tetraone $(8,50.0 \mathrm{mg}, 65.2 \mu \mathrm{mol})$ under argon atmosphere were dissolved in dichloromethane $(10 \mathrm{~mL})$, treated with second-generation Hoveyda-Grubbscatalyst $(3,7.6 \mathrm{mg}, 12 \mu \mathrm{mol})$, heated under reflux for $11 \mathrm{~h}$, stirred at room temperature for $12 \mathrm{~h}$, quenched by the addition of chloroform $(15 \mathrm{~mL})$, distilled water $(10 \mathrm{~mL})$ and acetic acid $(15 \mathrm{~mL})$, stirred for $2 \mathrm{~h}$, shaken three times with distilled water $(25 \mathrm{~mL}$ each), evaporated and purified by medium pressure chromatography (silica gel, chloroform/ethanol 60:1 at $35 \mathrm{~mL} \cdot \mathrm{min}^{-1}$, red fraction, column $36 \times 460 \mathrm{~mm}$ and a second medium pressure chromatography. Yield $3.0 \mathrm{mg}(3 \%)$ red solid. $R_{\mathrm{f}}$-value (silica gel, $\left.\mathrm{CHCl}_{3} / \mathrm{EtOH} 40: 1\right)=0.17$. UV/Vis $\left(\mathrm{CHCl}_{3}\right): \lambda_{\max }(E)=461.6(0.29), 491.8(0.75), 527.4 \mathrm{~nm}(1.00)$. Fluorescence $\left(\mathrm{CHCl}_{3}\right): \lambda_{\max }(I)=536.8(1.00), 579.8 \mathrm{~nm}(0.74) . \mathrm{MS}\left(\mathrm{DEI}^{+} / 70 \mathrm{eV}\right)$ : $m / z$ (\%): 1504 (2) $\left[M^{+}\right], 711(74)\left[M^{+}-\mathrm{C}_{53} \mathrm{H}_{65} \mathrm{~N}_{2} \mathrm{O}_{4}\right], 404(86)\left[M^{+}-\mathrm{C}_{62} \mathrm{H}_{81} \mathrm{~N}_{2} \mathrm{O}_{4}\right.$ $\left.-\mathrm{C}_{13} \mathrm{H}_{27}\right], 390$ (100) [404- $\mathrm{CH}_{2}$ ].

2-(1-Hexylheptyl)-9-\{1-(2,2,9-tributyl-9-((1-hexylheptyl)anthra[2,1,9-def ;6,5,10- $d^{\prime} e^{\prime} f$ ] diisoquinoline-1,3,8,10-tetraon)methyl)tridec-5-enyl\}anthra[ 2,1,9-def,6,5,10-d'e'f']diisoquinoline-1,3,8,10-tetraone(11):2-(1-Hexylheptyl) -9-(2,2-dibutylhept-6-enyl)-anthra[2,1,9-def,6,5,10-d'e'f] diiso-quinoline-1,3,8,10 -tetraone $(10,100 \mathrm{mg}, 0.128 \mathrm{mmol})$ under argon atmosphere was dissolved in tetrahydrofurane $(20 \mathrm{~mL})$, treated with second-generation Hoveyda-Grubbs-catalyst (3, $13 \mathrm{mg}, 21 \mu \mathrm{mol}$ ), stirred at room temperature for $18 \mathrm{~h}$, precipitated with 
methanol, collected by vacuum filtration, dried at $110^{\circ} \mathrm{C}$ in air and purifid by column separation (silica gel, chloroform). A pure fraction again forms a spectrum of side products. MS (ESI $\left.{ }^{+} / 70 \mathrm{eV}\right): \mathrm{m} / z$ (\%): 1091 (23), 915 (16), 457 (100).

2,9-Bis-\{2,9-bis-([2,2,9,9-tetrabutyl-dec-5-en-10-yl]yl)anthra[2,1,9-def,6, 5,10-d'e'f] diisoquinoline-1,3,8,10-tetraone $\}$ anthra[2,1,9-def,6,5,10-d'e'f] dii soquinoline-1,3,8,10-tetraone(22):2,9-Bis-(2,2-dibutyl-hex-5-enyl)anthra[2,1,9def;6,5,10-d'e'f] diisoquinoline-1,3,8,10-tetraone (21, $100 \mathrm{mg}, 128 \mu \mathrm{mol})$ was dissolved in warm dichloromethane $(100 \mathrm{~mL}$, clear solution)), treated with second-generation Hoveyda-Grubbs-catalyst (3, $16 \mathrm{mg}, 26 \mu \mathrm{mol}$, 1,3-bis-(2,4,6trimethylphenyl)-2-imidazolidinylidene)dichloro(2-isopropoxy-phenylmethyl phenylmethylene)ruthenium), stirred at $40^{\circ} \mathrm{C}$ for $5 \mathrm{~d}$, allowed to cool, precipitated with methanol (100 mL), collected by vacuum filtration (D4 glas filter), washed with methanol until colorless washings, dissolved in a minimal amount of chloroform, purified by medium pressure column chromatography $(500 \mathrm{~mL}$ of silica gel, chloroform/ethanol 60:1, second, red band), and precipitated with methanol. Yield $55.0 \mathrm{mg}(57 \%)$ dark red solid, m.p. $>250^{\circ} \mathrm{C}$. $R_{\mathrm{f}}$ value (silica gel; $\mathrm{CHCl}_{3} /$ EtOH 80:1) $=0.15$. IR $(\mathrm{KBr}): \tilde{v}=3502.2 \mathrm{w}$ br., $1953.8 \mathrm{~s}, 2927.6 \mathrm{~s}$, 2858.7 s, 1697.7 vs, 1656.6 vs, 1593.9 s, 1578.0 m, 1507.1 w, 1436.6 m, 1404.2 m, $1376.1 \mathrm{w}, 1332.3 \mathrm{~s}, 1250.2 \mathrm{~m}, 1217.0 \mathrm{w}, 1178.2 \mathrm{w}, 1159.3 \mathrm{w}, 1125.2 \mathrm{w}, 1015.8 \mathrm{w}$, $976.7 \mathrm{w}, 851.7 \mathrm{w}, 809.2 \mathrm{~m}, 795.8 \mathrm{w}, 747.0 \mathrm{~cm}^{-1} \mathrm{~m} .{ }^{1} \mathrm{H}$ NMR (600 MHz, $\mathrm{CDCl}_{3}$, $\left.25^{\circ} \mathrm{C}\right): \delta=0.84-1.75\left(\mathrm{~m}, 84 \mathrm{H}, 8 \mathrm{CH}_{3}+30 \mathrm{CH}_{2}\right), 2.25-2.39\left(\mathrm{~m}, 4 \mathrm{H}, 2 \mathrm{CH}_{2}\right)$, $3.85-4.42\left(\mathrm{~m}, 12 \mathrm{H}, 4 \mathrm{~N}-\mathrm{CH}_{2} \mathrm{R}+4 \mathrm{CH}_{\text {olefin. }}\right), 7.08-7.20\left(\mathrm{~m}, 4 \mathrm{H}, \mathrm{CH}_{\text {arom. }}\right)$, 7.52 $7.54\left(\mathrm{~m}, 2 \mathrm{H}, \mathrm{CH}_{\text {arom }}\right), 7.70-7.73\left(\mathrm{~m}, 2 \mathrm{H}, \mathrm{CH}_{\text {arom }}\right), 7.82-8.65 \mathrm{ppm}(\mathrm{m}, 8 \mathrm{H}$, $\mathrm{CH}_{\text {arom. }}$ ). UV $\mathrm{CHCl}_{3}: \lambda_{\max }(\varepsilon)=468.4(27300), 494.4(62800), 530.2 \mathrm{~nm}(56700)$. Fluorescence $\left(\mathrm{CHCl}_{3}\right): \lambda_{\max }\left(I_{\text {rel }}\right)=533.8(0.90), 582.8 \mathrm{~nm}$ (1.00). Fluorescence quantum yield ( $\lambda_{\text {exc }}=493 \mathrm{~nm}, E_{483 \mathrm{~nm} / 1 \mathrm{~cm}}=0.00997$, reference: 2,9-bis-(1-hexylheptyl) anthra[2,1,9-def, 6,5,10-d'e'f]diisoquinoline-1,3,8,10- tetraone (1) with $\Phi=1.00$

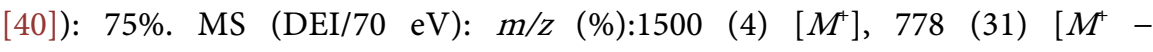
$\left.\mathrm{C}_{48} \mathrm{H}_{56} \mathrm{~N}_{2} \mathrm{O}_{4}\right], 723$ (37) $\left[M^{+}-\mathrm{C}_{52} \mathrm{H}_{60} \mathrm{~N}_{2} \mathrm{O}_{4}\right], 585(32)\left[M^{+}-\mathrm{C}_{48} \mathrm{H}_{56} \mathrm{~N}_{2} \mathrm{O}_{4}-\mathrm{C}_{14} \mathrm{H}_{27}\right]$, 418 (45) $\left[M^{+}-\mathrm{C}_{48} \mathrm{H}_{56} \mathrm{~N}_{2} \mathrm{O}_{4}-2 \times \mathrm{C}_{13} \mathrm{H}_{25}\right], 404(100)\left[M^{+}-\mathrm{C}_{48} \mathrm{H}_{56} \mathrm{~N}_{2} \mathrm{O}_{4}-\mathrm{C}_{13} \mathrm{H}_{25}\right.$ $\left.-\mathrm{C}_{14} \mathrm{H}_{27}\right], 391$ (57) $\left[M^{+}-\mathrm{C}_{48} \mathrm{H}_{56} \mathrm{~N}_{2} \mathrm{O}_{4}-2 \times \mathrm{C}_{14} \mathrm{H}_{27}\right]$. HRMS $\left(\mathrm{C}_{100} \mathrm{H}_{116} \mathrm{~N}_{4} \mathrm{O}_{8}\right)$ : Calcd. 1500.8794, found 1500.8788 .

2,9-Bis-\{2,9-bis-([2,2,7,7-tetrabutyloct-4-en-8-yl]yl)anthra[2,1,9-def,6,5,1 0-d'e'f] diisoquinoline-1,3,8,10-tetraone $\}$ anthra[2,1,9-def,6,5,10-d'e'f] diisoq uinoline-1,3,8,10-tetraone(20):2,9-Bis-(2-allyl-2-butylhexyl)anthra[2,1,9-def,6, 5,10-d'e'f] diisoquinoline-1,3,8,10-tetraone $(19,100 \mathrm{mg}, 133 \mu \mathrm{mol})$ under argon atmoshere was dissolved with warming in dry THF $(125 \mathrm{~mL})$, treated with second-generatio-Hoveyda-Grubbs catalyst (3,13 mg, $20 \mu \mathrm{mol} ; 15 \mathrm{~mol} \%$ ), stirred at $60^{\circ} \mathrm{C}$ for $18 \mathrm{~h}$ (orange fluorescent mixture); stirred at room temperarure for $31 \mathrm{~h}$, concentrated in vacou, recipitated with methanol, collected by vacuum filtration (D4 glas filter), dried at $110^{\circ} \mathrm{C}$, purified by column separation $(500 \mathrm{~mL}$ powder of silica gel, chloroform/ethanol 60:1, column, $500 \times 44 \mathrm{~mm}$ ) and purified by preparative TLC (silica gel, chloroform). Yield $48 \mathrm{mg}$ (50\%) dark red pigment, 
m.p. $>250^{\circ} \mathrm{C} . R_{\mathrm{f}}$-value (silica gel; $\left.\mathrm{CHCl}_{3} / \mathrm{EtOH} 60: 1\right)=0.24$. IR (ATR): $\tilde{v}=$ 2954.3 (m), 2927.9 (s), 2859.2 (m), 1697.4 (vs), 1654.8 (vs), 1593.5 (s), 1577.9 (m), $1507.1(\mathrm{w}), 1482.4(\mathrm{w}), 1455.6(\mathrm{w}), 1436.4(\mathrm{~m}), 1404.0(\mathrm{~m}), 1375.9(\mathrm{~m})$, 1332.6 (s), $1249.5(\mathrm{~m}), 1217.9(\mathrm{w}), 1177.3(\mathrm{w}), 1160.7(\mathrm{w}), 1101.8(\mathrm{w}), 1014.3$ $(\mathrm{w}), 972.0(\mathrm{w}), 850.4(\mathrm{w}), 809.1(\mathrm{~m}), 795.6(\mathrm{w}), 747.4(\mathrm{~m}), 671.9(\mathrm{w}), 638.0 \mathrm{~cm}^{-1}$ (w). UV/Vis $\left(\mathrm{CHCl}_{3}\right): \lambda_{\max }(E)=467.4$ (0.52) (sh.), $490.6(1.00), 527.4 \mathrm{~nm}(0.78)$. Fluorescence $\left(\mathrm{CHCl}_{3}\right): \lambda_{\max }(I)=529.8(0.21), 637.0 \mathrm{~nm}(1.00)$. MS: $\left(\mathrm{DEI}^{+} / 70\right.$ $\mathrm{eV}): m / z(\%): 1444(9)\left[M^{+}\right], 1265(3)\left[M^{+}-\mathrm{C}_{13} \mathrm{H}_{24}\right], 1252(3)\left[M^{+}-\mathrm{C}_{14} \mathrm{H}_{26}\right]$.

2,9-Bis-\{2,9-bis-([2,2,11,11-tetrabutyldodedec-6-en-12-yl]yl)anthra[2,1,9 -def,6,5,10-d'e'f] diisoquinoline-1,3,8,10-tetraone\}anthra[2,1,9-def,6,5,10-d' $\left.e^{\prime} f\right]$ diisoquinoline-1,3,8,10-tetraone:2,9-Bis-(2,2-dibutylhept-6-enyl)anthra[2, 1,9-def,6,5,10-d'e'f] diiso-quinoline-1,3,8,10-tetraone (100 mg, $124 \mu \mathrm{mol}$ ) under argon atmosphere was dissolved in dry THF $(150 \mathrm{~mL})$, treated with secondgeneration-Hoveyda-Grubs catalyst (3,10 mg, $16 \mu \mathrm{mol} ; 13 \mathrm{Mol} \%$ ), stirred at room temperature for $24 \mathrm{~h}$, concentrated in vacuo, precipitated with methanol, thoroughly washed with methanol, dried in air $\left(110^{\circ} \mathrm{C}, 16 \mathrm{~h}\right)$, purifid by column separation $(800 \mathrm{~mL}$ powder of silica gel, $800 \times 44 \mathrm{~mm}$ column, chloroform/ethanol 60:1) and purified by preparative TLC (silica gel, chloroform). Yield $67 \mathrm{mg}(69 \%)$ dark red pigment, m.p. $>250^{\circ} \mathrm{C} . R_{\mathrm{f}}$-value (silica gel; $\mathrm{CHCl}_{3} / \mathrm{EtOH}$ 60:1) = 0.24. IR (ATR): $\tilde{v}=2954.3(\mathrm{~s}), 2927.9$ (s), $2859.2(\mathrm{~m})$, 1697.4 (vs), 1654.8 (vs), 1593.6 (s), 1577.9 (m), 1507.1 (w), 1482.4 (w), 1455.6 $(\mathrm{w}), 1436.4(\mathrm{~m}), 1404.0(\mathrm{~m}), 1332.6(\mathrm{~s}), 1249.5(\mathrm{~m}), 1217.9(\mathrm{w}), 1177.3(\mathrm{w})$, $1160.7(\mathrm{w}), 1101.8(\mathrm{w}), 1014.3(\mathrm{w}), 972.0(\mathrm{w}), 912.1(\mathrm{w}), 850.4(\mathrm{w}), 809.1(\mathrm{~m})$, $795.6(\mathrm{w}), 747.4(\mathrm{~m}), 671.9(\mathrm{w}), 638.0 \mathrm{~cm}^{-1}(\mathrm{w}) . \mathrm{UV} / \mathrm{Vis}\left(\mathrm{CHCl}_{3}\right): \lambda_{\max }(E)=465.4$ (0.48) (sh.), 490.6 (1.00), $527.6 \mathrm{~nm}$ (0.89). Fluorescence $\left(\mathrm{CHCl}_{3}\right): \lambda_{\max }(I)=536.0$ $(0.11), 638.3 \mathrm{~nm}(1.00)$. Fluorescence quantum yield $\left(\lambda_{\mathrm{exc}}=493 \mathrm{~nm}, E_{493 \mathrm{~m} / 1 \mathrm{~cm}}=\right.$ 0.0100, reference: 2,9-bis-(1-hexylheptyl)anthra[2,1,9-def,6,5,10-d'e'f]-diisoquinoline-1,3,8,10-tetraone (1) with $\Phi=1.00$ [40]): 0.75. MS: $\left(\mathrm{DEI}^{+} / 70 \mathrm{eV}\right): \mathrm{m} / z$ (\%): 1557 (5) $\left[M^{+}\right], 543(5)\left[M^{+}-\mathrm{C}_{69} \mathrm{H}_{94} \mathrm{~N}_{2} \mathrm{O}_{4}\right], 529(6)\left[M^{+}-\mathrm{C}_{70} \mathrm{H}_{94} \mathrm{~N}_{2} \mathrm{O}_{4}\right]$.

2-But-3-enyl-2-hexyloctyl-9-\{2-but-3-enyl-2-hexyloctyl-9-([2,2,9,9-tetrab utyl-dedec-6-en-10-yl]yl)anthra[2,1,9-def,6,5,10-d'e'f] diisoquinoline-1,3,8, 10-tetraone $\}$ anthra $\left[2,1,9-d e f, 6,5,10-d^{\prime} e^{\prime} f\right]$ diisoquinoline-1,3,8,10-tetraone:2, 9-Bis-(2-but-3-enyl-2-hexyloctyl)anthra[2,1,9-def,6,5,10-d'e'f] diisoquinoline-1,3 ,8,10-tetraone $(50 \mathrm{mg}, 56 \mu \mathrm{mol})$ under argon atmosphere was dissolved in dry THF (175 mL), treated with second-generation Hoveyda-Grubbs-catalyst (3, 10 $\mathrm{mg}, 16 \mu \mathrm{mol} ; 13 \mathrm{Mol} \%$ ), stirred at room temperature for $24 \mathrm{~h}$, concentrated in vacuo, precipitated with methanol, collected by vacuum filtration, thoroughly washed with methanol, dried in air $\left(110^{\circ} \mathrm{C}, 16 \mathrm{~h}\right)$, purified by column separation (800 mL powder of silica gel, $800 \times 44 \mathrm{~mm}$ column, dichloromethane) and further purified by preparative TLC (silica gel, chloroform).

2-(2,2'-Ethoxyethan-diyl)-9-(1,12-(2,2,11,11-tetrabutyl)dodec-6-en-diyl) bis-anthra[2,1,9-def;6,5,10- $\left.d^{\prime} e^{\prime} f\right]$ diisoquinoline-1,3,8,10-tetraone(13):2-\{2(2,2-Dibutylhept-6-enyl)--9-(2-ethoxyethyl)anthra[2,1,9-def,6,5,10- $\left.d^{\prime} e^{\prime} f\right]$ diisoq 
uinoline-1,3,8,10-tetraon\}-9-(2,2-dibutylhept-6-enyl)anthra[2,1,9-def,6,5,10-d'e'f ] diisoquinoline-1,3,8,10-tetraone (12,200 $\mathrm{mg}, 0.158 \mathrm{mmol})$, second-generation Hoveyda-Grubbs-catalyst (3, $20 \mathrm{mg}, 32 \mu \mathrm{mol} ; 20 \mathrm{Mol} \%)$ and THF $(250 \mathrm{~mL})$ were allowed to react as was described for 2,9-bis-\{2,9-bis-([2,2,11,11-tetrabutyldodedec6-en-12-yl]yl)anthra[2,1,9-def,6,5,10-d'e'f] diisoquinoline-1,3,8,10-tetraone $\}$ anth ra[2,1,9-def,6,5,10-d'e'f]diisoquinoline-1,3,8,10-tetraone, stirred at room temperature for $24 \mathrm{~h}$ and purified by column separation ( $800 \mathrm{~mL}$ of silica gel, chloroform/ ethanol 15:1, glass column $800 \times 44 \mathrm{~mm}$ ) and further by medium pressure chromatography (silica gel, chloroform, column $36 \times 460 \mathrm{~mm}, 25 \mathrm{~mL} \cdot \mathrm{min}^{-1}$ ). Yield $10 \mathrm{mg}(10 \%)$ dark red pigment, m.p. $>250^{\circ} \mathrm{C} . R_{\mathrm{f}}$-value (silica gel; $\mathrm{CHCl}_{3} / \mathrm{EtOH}$ 10:1) = 0.54. IR (ATR): $\tilde{v}=3479.4$ (m,br.), 2952.3 (s), 2930.3 (s), 2865.1 (m), 1693.2 (vs), 1598.4 (vs), 1593.3 (vs), 1577.4 (s), 1507.6 (w), 1438.4 (m), $1403.9(\mathrm{~m}), 1336.0(\mathrm{~s}), 1247.4(\mathrm{~m}), 1167.8(\mathrm{w}), 1126.0(\mathrm{w}), 1054.2(\mathrm{w}), 852.8$ (w), $808.5(\mathrm{~m}), 794.3(\mathrm{w}), 744.4(\mathrm{~m}), 668.0(\mathrm{w}), 641.1 \mathrm{~cm}^{-1}(\mathrm{w})$. UV/Vis $\left(\mathrm{CHCl}_{3}\right)$ : $\lambda_{\max }(E)=466.6(0.44), 493.8(1.00), 529.8 \mathrm{~nm}(0.84)$. Fluorescence $\left(\mathrm{CHCl}_{3}\right): \lambda_{\max }$ $($ I $)=537.5$ (0.70), 584.5 (0.77), $628.5 \mathrm{~nm}$ (1.00). MS: $\left(\mathrm{DEI}^{+} / 70 \mathrm{eV}\right): \mathrm{m} / z(\%):$ $1223(<1)\left[M^{+}-\mathrm{CH}_{3}\right], 1182(<1)\left[M^{+}-\mathrm{C}_{4} \mathrm{H}_{9}\right], 530(10)\left[\mathrm{C}_{34} \mathrm{H}_{30} \mathrm{~N}_{2} \mathrm{O}_{4}\right], 460$ (13) $\left[\mathrm{C}_{28} \mathrm{H}_{16} \mathrm{~N}_{2} \mathrm{O}_{5}\right], 432(58)\left[\mathrm{C}_{26} \mathrm{H}_{12} \mathrm{~N}_{2} \mathrm{O}_{5}\right], 404$ (100) $\left[\mathrm{C}_{25} \mathrm{H}_{10} \mathrm{~N}_{2} \mathrm{O}_{4}\right], 390$ (88) $\left[\mathrm{C}_{24} \mathrm{H}_{8} \mathrm{~N}_{2} \mathrm{O}_{4}\right]$.

2-(1,8-(2,2-Dibutyl)oct-6-en-diyl)-9-(1,8-(7,7-dibutyl)oct-2-en-diyl)bis-a nthra[2,1,9-def,6,5,10-d' $\left.e^{\prime} f\right]$ diisoquinoline-1,3,8,10-tetraone (15) and 2-(1,4but-2-en-diyl)-9-(1,12-(2,2,11,11-tetrabutyl)dodec-6-en-diyl)bisanthra[2,1, 9-def,6,5,10-d' $e^{\prime} f$ ] diisoquinoline-1,3,8,10-tetraone(16):2-Allyl-9-(2,2-dib utyl-hept-6-enyl)anthra[2,1,9-def; 6,5,10-d'e'f] diisoquinoline-1,3,8,10-tetraone (14, $100 \mathrm{mg}, 0.157 \mathrm{mmol}$ ), second-generation Hoveyda-Grubbs-catalyst (3, 20 $\mathrm{mg}, 32 \mu \mathrm{mol}, 20 \mathrm{Mol} \%)$ and THF $(150 \mathrm{~mL})$ were allowed to react as was described for 2,9-bis-\{2,9-bis-([2,2,11,11-tetrabutyldodedec-6-en-12-yl]yl) anthra[2,1,9-def; 6,5,10-d'e'f] diisoquinoline-1,3,8,10-tetraone $\}$ anthra[2,1,9-def,6,5,10-d'e'f] diisoq uinoline-1,3,8,10-tetraone, stirred at room temperature for $24 \mathrm{~h}$ and purified by column separation (silica gel, chloroform). Yield $17 \mathrm{mg}$ (17\%) of a mixture of both isomers, m.p. $>250^{\circ} \mathrm{C}$. IR (ATR): $\tilde{v}=2952.7(\mathrm{~m}), 2930.2(\mathrm{~m}), 2862.7$ (m), 1696.8 (vs), 1653.8 (vs), 1593.3 (vs), 1578.0 (m), 1507.0 (w), 1482.1 (w), $1436.0(\mathrm{~m}), 1403.7(\mathrm{~m}), 1331.08 \mathrm{~m}), 1248.1(\mathrm{~m}), 1218.3(\mathrm{w}), 1195.8(\mathrm{w}), 1173.4$ (w), 1125.4 (w), $1095.3(w), 1012.1(w), 974.0(w), 927.1(w), 886.4(w), 851.3$ (w), $808.8(\mathrm{~m}), 795.3(\mathrm{w}), 746.0(\mathrm{~m}), 641.6 \mathrm{~cm}^{-1}(\mathrm{w})$. The isomers were separated by chromatography by means of fine silica gel $(800 \mathrm{~mL}$ of silica gel 40 to $60 \mu \mathrm{m}$, chloroform/ethanol 20:1, glasss column $800 \times 44 \mathrm{~mm})$. Isomer I: $(2-(1,8-(2,2-$ Dibutyl)oct-6-en-diyl)-9-(1,8-(7,7-dibutyl)oct-2-en-diyl)bis-anthra[2,1,9-def;6,5, 10- $d^{\prime} e^{\prime} f$ ]diisoquinoline-1,3,8,10-tetraone): Yield $5 \mathrm{mg}$ (5\%) dark red podwer. $R_{\mathrm{f}}$-value (silica gel; $\left.\mathrm{CHCl}_{3} / \mathrm{EtOH} 20: 1\right)=0.46$. UV/Vis $\left(\mathrm{CHCl}_{3}\right): \lambda_{\max }(E)=492.2$ (1.00), $528.8 \mathrm{~nm}(0.80)$. Fluorescence $\left(\mathrm{CHCl}_{3}\right): \lambda_{\max }(I)=534.5(0.31), 628.0 \mathrm{~nm}$ (1.00). Fluorescence quantum yield $\left(\lambda_{\text {exc }}=491 \mathrm{~nm}, E_{491 \mathrm{~nm} / 1 \mathrm{~cm}}=0.0126 \mathrm{~cm}^{-1}\right.$, reference: 2,9-bis-(1-hexylheptyl)anthra[2,1,9-def,6,5,10-d'eff] diisoquinoline- 1,3,8,10- 
tetraone (1) with $\Phi=1.00$ [40]): 0.69. MS: $\left(\mathrm{DEI}^{+} / 70 \mathrm{eV}\right): \mathrm{m} / z(\%): 1220(<1)$ $\left[M^{+}\right], 1182(<1)\left[M^{+}-\mathrm{C}_{3} \mathrm{H}_{4}\right], 1138(<1)\left[M^{+}-2 \times \mathrm{C}_{3} \mathrm{H}_{7}\right], 443(18)\left[\mathrm{C}_{28} \mathrm{H}_{14} \mathrm{~N}_{2} \mathrm{O}_{4}\right]$, 404 (85) $\left[M^{+}-\mathrm{C}_{25} \mathrm{H}_{12} \mathrm{~N}_{2} \mathrm{O}_{4}\right], 391$ (100) $\left[\mathrm{C}_{24} \mathrm{H}_{10} \mathrm{~N}_{2} \mathrm{O}_{4}\right]$. Isomer II: (2-(1,4-But2-en-diyl)-9-(1,12-(2,2,11,11-tetrabutyl)dodec-6-en-diyl)bisanthra[2,1,9-def;6,5, $10-d^{\prime} e^{\prime} f$ ]diisoquinoline-1,3,8,10-tetraone): Yield $5 \mathrm{mg}(5 \%)$ dark red powder. $R_{\mathrm{f}}$-value (silica gel; $\left.\mathrm{CHCl}_{3} / \mathrm{EtOH} 20: 1\right)=0.29$. UV/Vis $\left(\mathrm{CHCl}_{3}\right): \lambda_{\max }(E)=492.0$ (1.00), $528.8 \mathrm{~nm}(0.78)$. Fluorescence $\left(\mathrm{CHCl}_{3}\right): \lambda_{\max }(I)=535.0(0.36), 630.5 \mathrm{~nm}$ (1.00). Fluorescence quantum yield $\left(\lambda_{\mathrm{exc}}=491 \mathrm{~nm}, E_{491 \mathrm{~nm} / 1 \mathrm{~cm}}=0.0154 \mathrm{~cm}^{-1}\right.$, reference: 2,9-bis-(1-hexylheptyl)anthra-[2,1,9-def,6,5,10-d'e'f] diisoqui-noline1,3,8,10-tetraone (1) with $\Phi=1.00$ [13]): 0.88. MS: $\left(\mathrm{DEI}^{+} / 70 \mathrm{eV}\right): \mathrm{m} / z(\%): 1220$ (1) $\left[M^{+}\right], 1138(1)\left[M^{+}-2 \times \mathrm{C}_{3} \mathrm{H}_{7}\right], 443(20)\left[\mathrm{C}_{28} \mathrm{H}_{14} \mathrm{~N}_{2} \mathrm{O}_{4}\right], 404$ (82) $\left[M^{+}-\right.$ $\left.\mathrm{C}_{25} \mathrm{H}_{12} \mathrm{~N}_{2} \mathrm{O}_{4}\right], 391(100)\left[\mathrm{C}_{24} \mathrm{H}_{10} \mathrm{~N}_{2} \mathrm{O}_{4}\right]$.

\section{Acknowledgements}

We thank the Fonds der Chemischen Industrie for financial support.

\section{Conflicts of Interest}

The authors declare no conflicts of interest regarding the publication of this paper.

\section{References}

[1] Carroll, R.L. and Gorman, C.B. (2002) The Genesis of Molecular Electronics. Angewandte Chemie International Edition, 41, 4378-4400. https://doi.org/10.1002/1521-3773(20021202)41:23<4378::AID-ANIE4378>3.0.CO;2 $\underline{-\mathrm{A}}$

[2] Langhals, H. (2005) Control of the Interactions in Multichromophores: Novel Concepts. Perylene Bisimides as Components for Larger Functional Units. Helvetica Chimia Acta, 88, 1309-1343. https://doi.org/10.1002/hlca.200590107

[3] Langhals, H. (2018) Homologous of S-13: Highly Fluorescent and Soluble Dyes. https://www.cup.lmu.de/oc/langhals/Perylenebiscarboximides/index.html

[4] Langhals, H. (2014) Handling Electromagnetic Radiation beyond Terahertz Using Chromophores to Transition from Visible Light to Petahertz Technology. Jounal of Electric \& Electronic Systems, 3, 125. https://doi.org/10.4172/2332-0796.1000125

[5] Davydov, A.S. (1948) Theory of Absorption Spectra of Molecular Crystals. Theory of Absorption Spectra of Molecular Crystals, 18, 210-218. https://doi.org/10.1136/jech.3.1.43

[6] Davydow, A.S. (1962) Theory of Molecular Excitations. Transl. Kasha, H. and Oppenheimer Jr., M., McGraw-Hill, New York.

[7] Scheibe, G. (1936) Variability of the Absorption Spectra of Some Sensitizing Dyes and Its Cause. Angewandte Chemie, 49, 563.

[8] Jelley, E.E. (1936) Spectral Absorption and Fluorescence of Dyes in the Molecular State. Nature, 138, 1009-1010. https://doi.org/10.1038/1381009a0

[9] Förster, T. (1946) Energiewanderung und Fluoreszenz. Naturwissenschaften, 33, 166-175. https://doi.org/10.1007/BF00585226

[10] Langhals, H. and Ismael, R. (1998) Cyclophanes as Model Compounds for Permanent, Dynamic Aggregates-Induced Chirality with Strong CD Effects. European Journals 
of Organic Chemistry, 1998, 1915-1917.

https://doi.org/10.1002/(SICI)1099-0690(199809)1998:9<1915::AID-EJOC1915>3.0. $\mathrm{CO} ; 2-1$

[11] Grubbs, R.H. and Chang, S. (1998) Recent Advances in Olefin Metathesis and Its Application in Organic Synthesis. Tetrahedron, 54, 4413-4450.

https://doi.org/10.1016/S0040-4020(97)10427-6

[12] Fürstner, A. (2000) Olefin Metathesis and beyond. Angewandte Chemie International Edition, 39, 3012-3043. https://doi.org/10.1002/1521-3773(20000901)39:17<3012::AID-ANIE3012>3.0.CO;2-G

[13] Calderon, N. (1972) Olefin Metathesis Reaction. Accounts of Chemical Research, 5, 127-132. https://doi.org/10.1021/ar50052a002

[14] Schuster, S. and Blechert, S. (1997) Olefin Metathesis in Organic Chemistry. Angewandte Chemie International Edition, 36, 2036-2056. https://doi.org/10.1002/anie.199720361

[15] Watson, M.D., Jäckel, F., Severin, N., Rabe, J.P. and Müllen, K. (2004) A Hexaperi-Hexabenzocoronene Cyclophane: An Addition to the Toolbox for Molecular Electronics. Jounal of the American Chemical Society, 126, 1402-1407. https://doi.org/10.1021/ja037520p

[16] Langhals, H., Demmig, S. and Huber, H. (1988) Rotational Barriers in Perylene Fluorescent Dyes. Spectrochimica Acta A, 44, 1189-1193. https://doi.org/10.1016/0584-8539(88)80091-6

[17] Garber, J.S.B., Kingsbury, S., Gray, B.L. and Hoveyda, A.H. (2000) Efficient and Recyclable Monomeric and Dendritic Ru-Based Metathesis Catalysts. Journal of the American Chemical Society, 122, 8168-8179. https://doi.org/10.1021/ja001179g

[18] Langhals, H. and Bastani-Oskoui, H. (1997) Synthesis of Readily Soluble Tetraazaviolanthrone and Isoviolanthrone Fluorescent Dyes. Journal für Praktische Chemie, 339, 597-602. https://doi.org/10.1002/prac.199733901107

[19] Langhals, H., Ismael, R. and Yürük, O. (2000) Persistent Fluorescence of Perylene Dyes by Steric Inhibition of Aggregation. Tetrahedron, 56, 5435-5441.

https://doi.org/10.1016/S0040-4020(00)00452-X

[20] Langhals, H. and Rüchardt, C. (1981) Wanderungstendenzen cyclischer, polycyclischer und methylverzweigter Alkylreste bei der Beckmann-Umlagerung. Chemische Berichte, 114, 3831-3854. https://doi.org/10.1002/cber.19811141208

[21] Missling, C.U., Sünkel, K., Langhals, H. and Beck, W. (2017) Organometallic Lewis Acids, Part CXII. Chiral Carbonyl-Cyclopentadienyl-Triphenylphosphine-Iron and -Ruthenium Complexes with Novel Tertiary Nitriles. $\left[\mathrm{CpM}(\mathrm{CO})\left(\mathrm{PPh}_{3}\right)\left(\mathrm{N} \equiv \mathrm{C}-\mathrm{CR}_{1} \mathrm{R}_{2} \mathrm{R}_{3}\right)\right]^{+}$ $\mathrm{BF}_{4}^{-}(\mathrm{M}=\mathrm{Fe}, \mathrm{Ru})$. Zeitschrift für anorganische und allgemeine Chemie, 643, 1262-1268. https://doi.org/10.1002/zaac.201700235

[22] Langhals, H. (1985) Synthese von hochreinen Perylen-Fluoreszenzfarbstoffen in großen Mengen-gezielte Darstellung von Atrop-Isomeren. Chemische Berichte, 118, 4641-4645. https://doi.org/10.1002/cber.19851181138

[23] Langhals, H., Demmig, S. and Potrawa, T. (1991) The Relation between Packing Effects and Solid State Fluorescence of Dyes. Journal für Praktishe Chemie, 333, 733-748. https://doi.org/10.1002/prac.19913330508

[24] Langhals, H. and Jona, W. (1998) Intense Dyes through Chromophore-Chromophore Interactions: Bi- and Trichromophoric Perylene-3,4:9,10-bis(dicarboximide)s. Angewandze Chemie International Edition, 37, 952-955.

https://doi.org/10.1002/(SICI)1521-3773(19980420)37:7<952::AID-ANIE952>3.0.C O;2-4 
[25] Langhals, H. and Gold, J. (1996) Tangentially Coupled $\pi$ Systems and Their Through-Space Interaction-Trichromophoric Perylene Dyes. Journal für Praktische Chemie, 338, 654-659. https://doi.org/10.1002/prac.199633801124

[26] Langhals, H. and Pust, T. (2011) Lipophilic Optical Supramolecular Nano Devices in the Aqueous Phase. Green and Sustainable Chemistry, 1, 1-6. https://doi.org/10.4236/gsc.2011.11001

[27] Feng, J., Zhang, Y., Zhao, C., Li, R., Xu, W., Li, X. and Jiang, J. (2008) Cyclophanes of Perylene Tetracarboxylic Diimide with Different Substituents at Bay Positions. Chemistry: An European Journal, 14, 7000-7010. https://doi.org/10.1002/chem.200800136

[28] Schlosser, F., Moos, M., Lamber, C. and Würthner, F. (2013) Redox-Switchable Intramolecular $\pi$ - $\pi$-Stacking of Perylene Bisimide Dyes in a Cyclophane. Advanced Materials, 25, 410-414. https://doi.org/10.1002/adma.201201266

[29] Spenst, P. and Wîrthner, F. (2015) A Perylene Bisimide Cyclophane as a "Turn-On” and “Turn-Off” Fluorescence Probe. Angewandte Chemie International Edition, 54, 10165-10168. https://doi.org/10.1002/anie.201503542

[30] Spenst, P., Young, R.M., Wasielewski, M.R. and Würthner, F. (2016) Guest and Solvent Modulated Photo-Driven Charge Separation and Triplet Generation in a Perylene Bisimide Cyclophane. Chemical Science, 7, 5428-5434. https://doi.org/10.1039/C6SC01574C

[31] Spenst, P., Sieblist, A. and Würthner, F. (2017) Perylene Bisimide Cyclophanes with High Binding Affinity for Large Planar Polycyclic Aromatic Hydrocarbons: Host-Guest Complexation versus Self-Encapsulation of Side Arms. Chemistry: An European Journal, 23, 1667-1675. https://doi.org/10.1002/chem.201604875

[32] Ruggli, P. (1913) Triple Bond Ring. Justus Liebigs Annalen der Chemie Banner, 392, 92-100. https://doi.org/10.1002/jlac.19123920106

[33] Ziegler, K. (1934) Ring-Closure Reactions. Berichte der Deutschen Chemischen Gesellschaft ( $A$ and B Series) Banner, 67, A139-A149. https://doi.org/10.1002/cber.19340671142

[34] Langhals, H. (1981) Prinzipielle Wege für die Gewinnung von Solarenergie über gezielte Modifizierung fluoreszierender Systeme.

[35] Baur, G., Götzberger, A., Heidler, K., Langhals, H., Sah, E., Wittwer, V. and Zastrow, A. (1982) Grundlagen der Solarenergieumwandlung auf der Basis von Fluoreszenzkollektoren.

[36] Sheldrick, G.M. (2015) Crystal Structure Refinement with SHELXL. Acta Crystallographica C, 71, 3-8.

[37] Farrugia, L.J. (2012) WinGX and ORTEP for Windows: An Update. Journal of Applied Crystallography, 45, 849-854. https://doi.org/10.1107/S0021889812029111

[38] Kaiser, H., Lindner, J. and Langhals, H. (1991) Synthesis of Nonsymmetrically Substituted Perylene Fluorescent Dyes. Chemische Berichte, 124, 529-535. https://doi.org/10.1002/cber.19911240319

[39] Tsuji, J., Yamada, T., Minami, I., Yuhara, M., Nisar, M. and Shimizu, I. (1987) Palladium-Catalyzed Decarboxylation-Allylation of Allylic Esters of $\alpha$-Substituted $\beta$-Keto Carboxylic, Malonic, Cyanoacetic, and Nitroacetic Acids. Journal of Organic Chemistry, 52, 2988-2995. https://doi.org/10.1021/jo00390a007

[40] Langhals, H., Karolin, J. and Johansson, L.B.-Å. (1998) Spectroscopic Properties of New and Convenient Standards for Measuring Fluorescence Quantum Yields. Journal of the Chemical Society, Faraday Transactions, 94, 2919-2922. https://doi.org/10.1039/a804973d 